Tôhoku Math. Journ. 20(1968), 516-553.

\title{
ON THE GENERALIZED WHITEHEAD PRODUCTS AND THE GENERALIZED HOPH INVARIANT OF A COMPOSITION ELEMENT
}

\author{
HIDEO ANDO
}

(Received April 30, 1968)

Introduction. In [11] $\mathrm{Hu}$ generalized the Whitehead product to the relative product, moreover in [4] Blaker-Massey defined the mixed Whitehead product in the relative homotopy groups and the product in the triad homotopy groups. Recently, Arkowitz [2], Hilton [10] and Porter [12] have defined the generalized Whitehead product (GWP) $[\alpha, \beta] \in \pi(\Sigma(A \# B), X)$, for $\alpha \in \pi(\Sigma A, X)$ and $\beta \in \pi(\Sigma B, X)$, and studied various properties of the GWP. In the case when $A$ and $B$ are spheres $[\alpha, \beta]$ is essentially the Whitehead product.

The object of this paper is to investigate operations which are generalizations of the Whitehead products in the relative homotopy groups and the triad homotopy groups. We define the following six new products in this paper :

$$
\begin{array}{lllll}
{[\alpha, \beta]_{1} \in \pi_{1}(\Sigma(A \# B), k)} & \text { for } & \alpha \in \pi_{1}(\Sigma A, k) \text { and } \beta \in \pi(\Sigma B, X), \\
{[\alpha, \beta]_{2} \in \pi_{1}(\Sigma(A \# B), k)} & \text { for } & \alpha \in \pi_{1}(\Sigma A, k) \text { and } \beta \in \pi_{1}(\Sigma B, k), \\
{[\alpha, \beta]_{3} \in \pi_{2}(\Sigma(A \# B), \Phi)} & \text { for } & \alpha \in \pi_{1}(\Sigma A, u) \text { and } \beta \in \pi_{1}(\Sigma B, v), \\
{[\alpha, \beta]_{4} \in \pi_{2}(\Sigma(A \# B), \Phi)} & \text { for } & \alpha \in \pi_{2}(\Sigma A, \Phi) \text { and } \beta \in \pi(\Sigma B, W), \\
{[\alpha, \beta]_{5} \in \pi_{2}(\Sigma(A \# B), \Phi)} & \text { for } & \alpha \in \pi_{2}(\Sigma A, \Phi) \text { a:d } \beta \in \pi_{1}(\Sigma B, u), \\
{[\alpha, \beta]_{6} \in \pi_{2}(\Sigma(A \# B), \Phi)} & \text { for } & \alpha \in \pi_{2}(\Sigma A, \Phi) \text { and } & \beta \in \pi_{2}(\Sigma B, \Phi),
\end{array}
$$

where $k: X \rightarrow Y$ is a map and $\Phi=\left(v, v^{\prime}\right): u \rightarrow u^{\prime}$ is a pair-map in which $u$ : $w \rightarrow X_{1}, v: W \rightarrow X_{2}, u^{\prime}: X_{2} \rightarrow X$ and $v^{\prime}: X_{1} \rightarrow X$.

If $A, B$ are spheres and $k$ is an inclusion map and $X_{1}, X_{2} \subset X, W=X_{1} \cap X_{2}$ then $[\alpha, \beta]_{1},[\alpha, \beta]_{2}$ and $[\alpha, \beta]_{3}$ are essentially the mixed Whitehead product, the relative and triad Whitehead product, respectively. In section 2 we define generalized Whitehead elements and in section 3 we define the above GWP's by using the generalized Whitehead elements, and basic properties of these products are mentioned in section 4 . The Jacobi identities of these products are obtained in section 5 . In section 6 we define the generalized Hopf 
invariant $H^{*}$ in the generalized homotopy groups by considering the Hopf invariant described in [1] and we generalize Theorem 1 in [8] .

Throughout this paper we shall assume that all spaces have base points and all maps (homotopies) are base point preserving and in section 1-5 spaces $A, B$ and $C$ are countable connected $C W$-complexes. In particular, in section 6 we shall assume that all spaces are finite $C W$-complexes.

1. Preliminaries. A map $q: X \rightarrow Y$ is called a cofibration if it has the homotopy lowering property for all spaces, i.e., if, for each space $P$ and for all maps $f_{0}: Y \rightarrow P$ and homotopies $g_{t}: X \rightarrow P(0 \leqq t \leqq 1)$ with $g_{0}=f_{0} \circ q$, there exists a homotopy $f_{t}: Y \rightarrow P$ with $g_{t}=f_{t} \circ q$. If $q$ is an inclusion map, this is homotopy extension property. The quotient space $F=Y / q(X)$ is called the cofibre of $q$. Frequently the cofibration $q: X \rightarrow Y$ with cofibre $F$ is denoted by the sequence $X \stackrel{q}{\longrightarrow} Y \stackrel{p}{\longrightarrow} F$, where $p$ is the projection.

The set of all homotopy classes of maps $X \rightarrow Y$ is denoted by $\pi(X, Y)$, it contains the distinguished element 0 , i.e., the homotopy class of the constant map $*: X \rightarrow Y$. A pair-map $\left(g_{1}, g_{2}\right): \iota_{V} \rightarrow f$ is by definition a map of maps such that the diagram

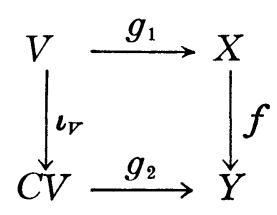

is commutative, where $\iota_{V}$ is the map $V \rightarrow C V$ which embeds $V$ in the cone $C V$. Then $\pi_{1}(V, f)$ is defined as the set of homotopy classes of $\left(g_{1}, g_{2}\right)$, and if $V$ is a suspension space (a 2-fold suspension space) $\pi_{1}(V, f)$ is a group (an abelian group). Moreover, if $f$ is an inclusion and $V=S^{n}, n \geqq 1$, we get the ordinary :alative homotopy groups. The homotopy class of a pair-map $\left(g_{1}, g_{2}\right)$ : $\iota_{V} \rightarrow f$ is denoted by $\left\{\left(g_{1}, g_{2}\right)\right\}$. A map $\left(\begin{array}{l}h_{1} h_{2} \\ h_{3} h_{4}\end{array}\right):\left(\iota_{V}, C_{\iota_{V}}\right) \rightarrow \Phi_{Z}$ is by definition a map of pair-maps such that the diagram

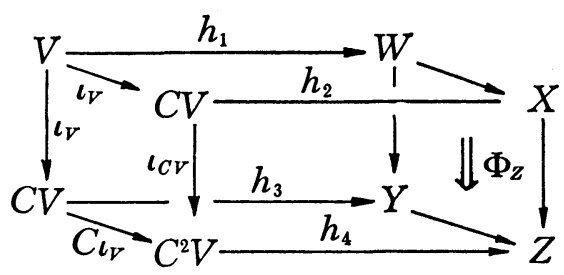

is commutative. Then $\pi_{2}\left(V, \Phi_{Z}\right)$ is defined as the set of homotopy classes of 
$\left(\begin{array}{l}h_{1} h_{2} \\ h_{3} h_{4}\end{array}\right)$, and if $V$ is a suspension space (a 2 -fold suspension space) $\pi_{2}\left(V, \Phi_{Z}\right)$ is a group (an abelian group) and a generalization of the triad homotopy groups. The homotopy class of $\left(\begin{array}{l}h_{1} h_{2} \\ h_{3} h_{4}\end{array}\right)$ is denoted by $\left\{\left(\begin{array}{l}h_{1} h_{2} \\ h_{3} h_{4}\end{array}\right)\right\}$.

The (reduced) suspension $\Sigma X$ of $X$ is the space obtained from $X \times I$ by identifying $X \times I \cup * \times I$ to a point. The (reduced) cone $C X$ of $X$ is the space obtained from $X \times I$ by identifying $X \times 0 \cup * \times I$ to a point. We denote by $X \vee Y$ the subspace $X \times * \cup * \times Y$ of $X \times Y$. The smash product $X \# Y$ of $X$ and $Y$ is the space obtained from $X \times Y$ by identifying $X \vee Y$ to a point. For maps $f: X \rightarrow Y$ and $g: X^{\prime} \rightarrow Y^{\prime}$, we define a map $f \vee g: X \vee X^{\prime} \rightarrow Y \vee Y^{\prime}$ by $f \vee g=f \times g \mid X \vee X^{\prime}$, and a map $f \# g: X \# X^{\prime} \rightarrow Y \# Y^{\prime}$ is defined by the following commutative diagram

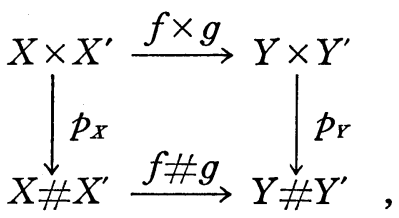

where $p$ 's are identification maps.

The following properties are checked easily:

(i) If $f \simeq f^{\prime}: X \rightarrow Y$ and $g \simeq g^{\prime}: X^{\prime} \rightarrow Y^{\prime}$ then

$$
f \# g \simeq f^{\prime} \# g^{\prime}: X \# X^{\prime} \rightarrow Y \# Y^{\prime} \text {. }
$$

$$
(f \# g) \# h=f \#(g \# h) \text {. }
$$

Let $f$ and $g$ be representatives of $\alpha \in \pi(X, Y)$ and $\beta \in \pi\left(X^{\prime}, Y^{\prime}\right)$ respectively. Then $f \# g$ is independent of the choice of $f$ and $g$ by (i) of (1.1) and the homotopy class is denoted by $\alpha \# \beta \in \pi\left(X \# X^{\prime}, Y \# Y^{\prime}\right)$. It follows from (1.1) that

$$
(\alpha \# \beta) \# \gamma=\alpha \#(\beta \# \gamma)
$$

Next we consider two elements $\alpha \in \pi(X, Y)$ and $\beta \in \pi(Y, Z)$ and let $\alpha=\{f\}$ and $\beta=\{g\}$. Then the composition $g \circ f: X \rightarrow Z$ of $f$ and $g$ represents an element of $\pi(X, Z)$ which is independent of the choice of the representatives $f$ and $g$, and the homotopy class of $g \circ f$ is denoted by $\beta \circ \alpha \in \pi(X, Z)$ and is called as the composition of $\alpha$ and $\beta$. The formula $\beta \circ \alpha=f^{*}(\beta)=g_{*}(\alpha)$ defines maps $f^{*}: \pi(Y, Z) \rightarrow \pi(X, Z)$ and $g_{*}: \pi(X, Y) \rightarrow \pi(X, Z)$ induced by $f$ and $g$ respectively. The join of $A$ and $B, A * B$ is the quotient space 
obtained from $A \times B \times I$ by factoring out the relation: $\left(a, b_{1}, 0\right) \sim\left(a, b_{2}, 0\right)$ for all $b_{1}, b_{2} \in B$ and $\left(a_{1}, b, 1\right) \sim\left(a_{2}, b, 1\right)$ for all $a_{1}, a_{2} \in A$.

Proposition (1.3) $A * B$ is homotopy equivalent to $Q=C A \times B \cup A \times C B$ [12].

Proposition (1.4) $A * B$ is homotopy equivalent to $\Sigma(A \# B)$ and there exists a hornotopy equivalence $\bar{h}_{A, B}: \Sigma(A \# B) \rightarrow Q$ ([10], [12]).

We denote by $\bar{\rho}_{A, B}: Q \rightarrow \Sigma A \vee \Sigma B$ the map which pinches $A \subset C A$ and $B \subset C B$ to * .

2. The generalized Whitehead elements. We consider the following pair-maps $\psi=\left(1_{\Sigma A} \bigvee \iota_{\Sigma B}, 1_{C \Sigma A} \vee \iota_{\Sigma B}\right): \iota_{\Sigma A} \bigvee 1_{\Sigma B} \rightarrow \iota_{\Sigma A} \bigvee 1_{C \Sigma B}, \Psi_{1}=\left(\iota_{\Sigma A} \vee 1_{\Sigma B}, \iota_{C \Sigma A} \bigvee 1_{\Sigma B}\right):$ $\iota_{\Sigma A} \vee 1_{\Sigma B} \rightarrow C \iota_{\Sigma A} \vee 1_{\Sigma B}, \quad \Psi_{2}=\left(\iota_{\Sigma A} \vee 1_{\Sigma B}, \iota_{C \Sigma A} \vee 1_{C \Sigma B}\right): \iota_{\Sigma A} \vee \iota_{\Sigma B} \rightarrow C \iota_{\Sigma A} \vee \iota_{\Sigma B}, \Psi_{3}$ $=\left(\iota_{\Sigma A} \vee \iota_{\Sigma B}, \iota_{C \Sigma A} \vee \iota_{C \Sigma B}\right): \iota_{\Sigma A} \vee \iota_{\Sigma B} \rightarrow C \iota_{\Sigma A} \vee C_{\iota_{\Sigma B}}$
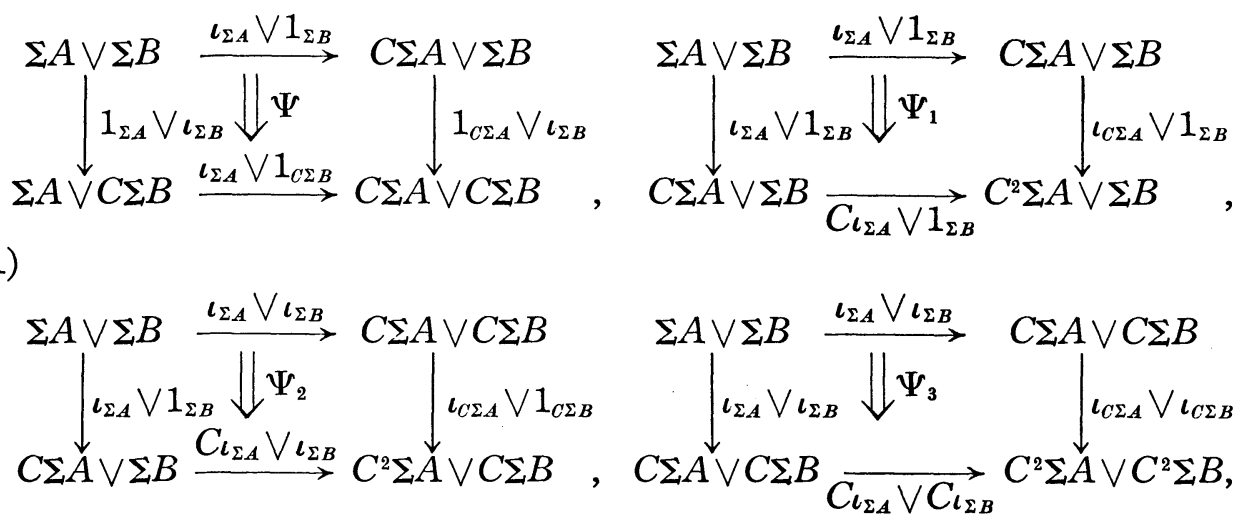

where $1_{\Sigma B}\left(1_{\Sigma B}\right): \Sigma A(\Sigma B) \rightarrow \Sigma A(\Sigma B), 1_{C \Sigma A}\left(1_{C \Sigma B}\right): C \Sigma A(C \Sigma B) \rightarrow C \Sigma A(C \Sigma B)$ and $1_{C^{2} \Sigma A}: C^{2} \Sigma A \rightarrow C^{2} \Sigma B$ are identity maps, and $\iota_{\Sigma A}\left(\iota_{\Sigma B}\right): \Sigma A(\Sigma B) \subset C \Sigma A(C \Sigma B)$, $\iota_{C \Sigma A}\left(\iota_{C \Sigma B}\right): C \Sigma A(C \Sigma B) \subset C^{2} \Sigma A\left(C^{2} \Sigma B\right), C_{\iota_{\Sigma A}}\left(C_{\iota_{\Sigma B}}\right): C \Sigma A(C \Sigma B) \subset C^{2} \Sigma A\left(C^{2} \Sigma B\right)$.

Then we have the following commutative diagrams

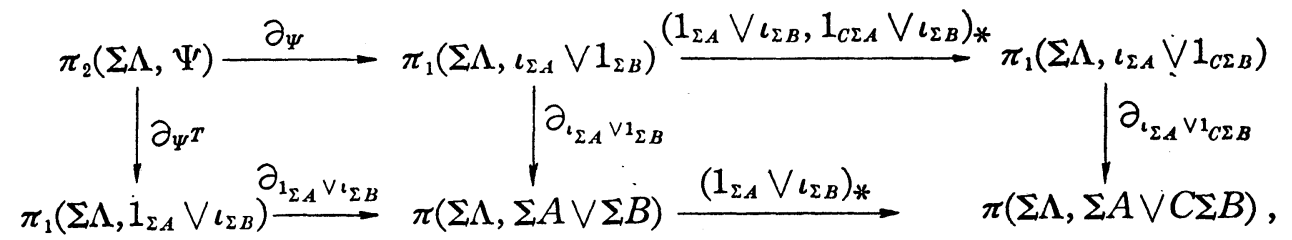


$(2.1 .2)$
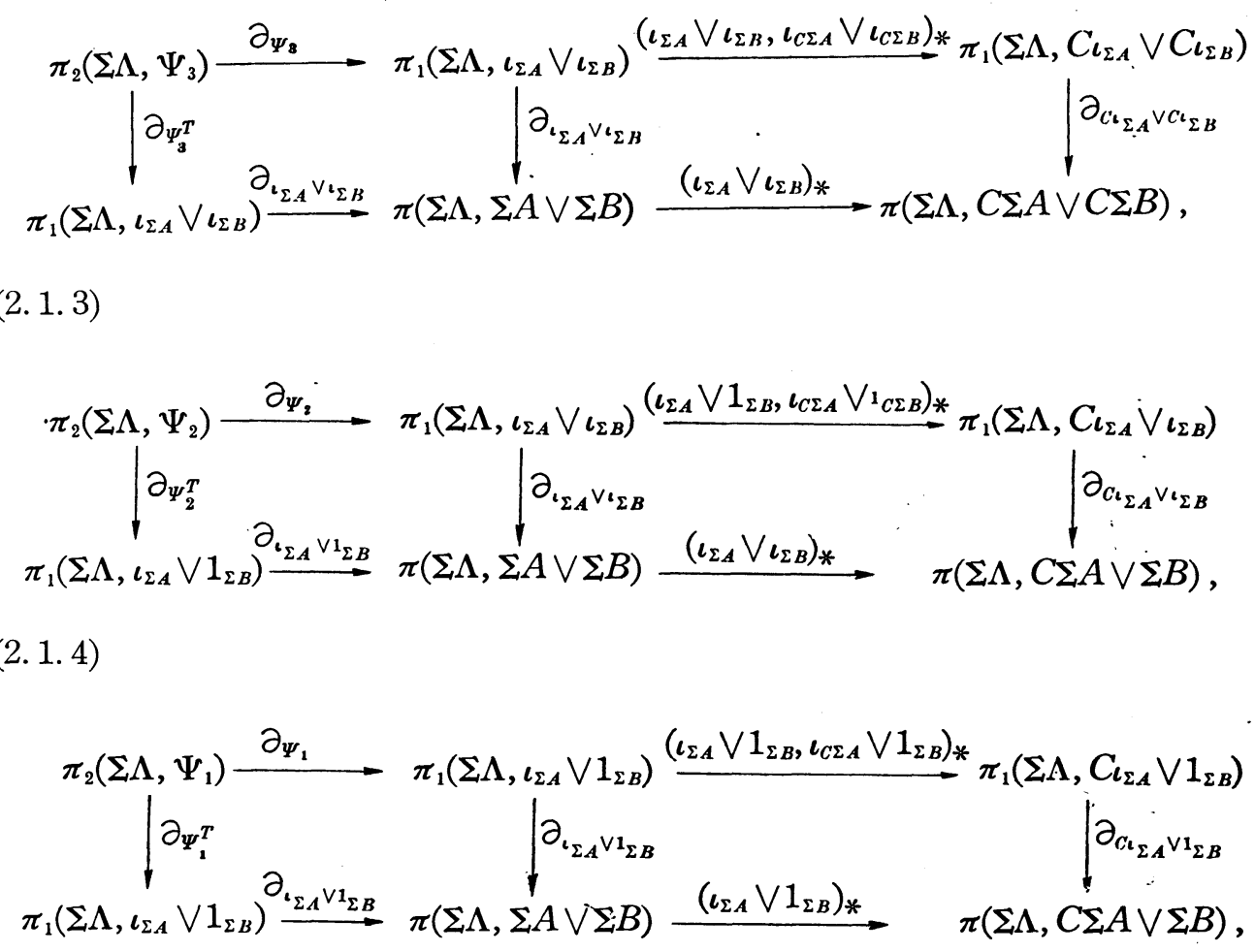

in which $\partial$ 's are the boundary homomorphisms (c.f. [5], [10]) and $\Sigma \Lambda=\Sigma(A \# B)$, and $\pi_{2}(\Sigma \Lambda, \Psi)$ and $\pi_{2}\left(\Sigma \Lambda, \Psi_{i}\right)(i=1,2,3)$ are identified with $\pi_{2}\left(\Sigma \Lambda, \Psi^{T}\right)$ and $\pi_{2}\left(\Sigma \Lambda, \Psi_{i}^{T}\right)$ respectively, under the isomorphism $\tau$ defined in [5, p. 291]. Then we see

PROPOSITION (2.2) (i) $\partial_{\iota_{\Sigma A} \vee \iota_{\Sigma B}}, \partial_{\iota_{\Sigma A} \vee 1_{C \Sigma B}}, \partial_{c_{\iota_{\Sigma A}} v_{\iota_{\Sigma B}}}, \partial_{c_{\iota_{\Sigma A}} \vee 1_{\Sigma B}}, \partial_{\Psi_{3}}$ and $\partial_{\Psi_{z}^{T}}$ are isomorphisms, (ii) (1 $\left.1_{\Sigma A} \vee \iota_{\Sigma B}\right)_{*},\left(\iota_{\Sigma A} \bigvee 1_{\Sigma B}\right)_{*},\left(1_{\Sigma A} \bigvee \iota_{\Sigma B}, 1_{C \Sigma_{A}} \vee \iota_{\Sigma_{B}}\right)_{*},\left(\iota_{\Sigma A} \vee 1_{\Sigma B}\right.$, $\left.\iota_{C \Sigma A} \vee 1_{C \Sigma B}\right)_{*}$ and $\left(\iota_{\Sigma A} \vee 1_{\Sigma B}, \iota_{C \Sigma A} \vee 1_{\Sigma B}\right)_{*}$ are epimorphisms, (iii) $\partial_{1_{\Sigma A} \vee \iota_{\Sigma B}}, \partial_{\iota_{\Sigma A} \vee 1_{\Sigma B}}$, $\partial_{\Psi}, \partial_{\Psi_{1}}$ and $\partial_{\Psi_{2}}$ are monomorphisms.

ProOF. (i) Since $\pi(\Sigma \Lambda, C \Sigma A \vee C \Sigma B)=0$ in the homotopy exact sæquence of the map $\iota_{\Sigma A} \vee \iota_{\Sigma B}, \partial_{\iota_{\Sigma A} \vee \iota_{\Sigma B}}$ is an isomorphism. Similarly, the other boundary homomorphisms are isomorphisms. (ii) Let $\left(i_{\Sigma A}, i_{C \Sigma A}\right)$ be a pair-map : $\iota_{\Sigma A}$ $\rightarrow \iota_{\Sigma A} \vee 1_{\Sigma B}$, where $i_{\Sigma A}: \Sigma A \subset \Sigma A \vee \Sigma B$ and $i_{C \Sigma A}: C \Sigma A \subset C \Sigma A \vee \Sigma B$ and $\iota_{\Sigma A}$ : $\Sigma A \subset C \Sigma A$. Then we consider the following commutative diagram 


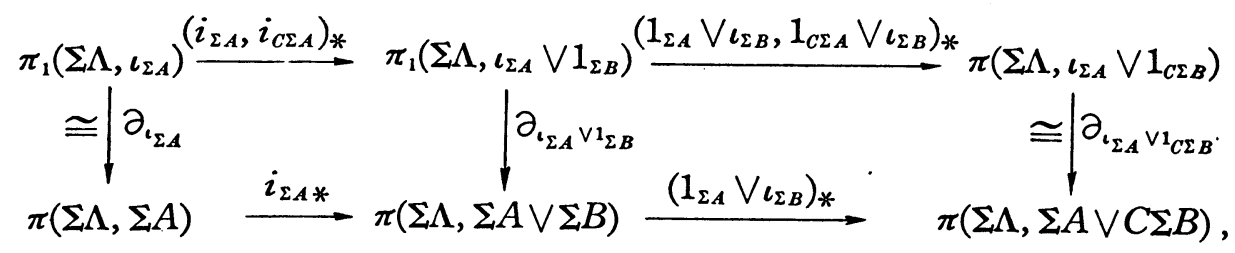

$\left(\left(1_{\Sigma A} \vee \iota_{\Sigma B}\right) \circ i_{\Sigma A}\right)_{*}=\left(1_{\Sigma A} \vee \iota_{\Sigma B}\right)_{*} \circ i_{\Sigma A *}$ is an epimorphism if and only if $\left(1_{\Sigma A} \vee \iota_{\Sigma B}\right.$, $\left.1_{C \Sigma A} \vee \iota_{\Sigma B}\right)_{*} \circ\left(i_{\Sigma A}, i_{C \Sigma A}\right)_{*}$ is an epimorphism, and hence $\left(1_{\Sigma_{A}} \vee_{\iota_{\Sigma B}}, 1_{C \Sigma A} \vee \iota_{\Sigma B}\right)_{*}$ is an epimorphism. Also since $\left(1_{\Sigma A} \vee \iota_{\Sigma B}\right)_{*} i_{\Sigma A *}$ is an epimorphism we deduce that $\left(1_{\Sigma A} \vee \iota_{\Sigma B}\right)_{*}$ is an epimorphism. Similarly, the other homomorphisms are epimorphisms. (iii) is obvious.

Next we define the generalized Whitehead elements.

(a) Let $\bar{h}_{A, B}: \Sigma(A \# B) \rightarrow Q_{A, B}=C A \times B \cup A \times C B$ and $\bar{\rho}_{A, B}: Q_{A, B} \rightarrow \Sigma A \vee \Sigma B$ be the maps defined in section 1 (c.f. [12]). Then we define $\theta$ by

$$
\theta=\left\{\bar{\rho}_{A, B} \bar{h}_{A, B}\right\} \in \pi(\Sigma(A \# B), \Sigma B \vee \Sigma B),
$$

where $\bar{h}_{A, B}$ is the homotopy equivalence described in (1.4).

(b) Consider a commutative diagram

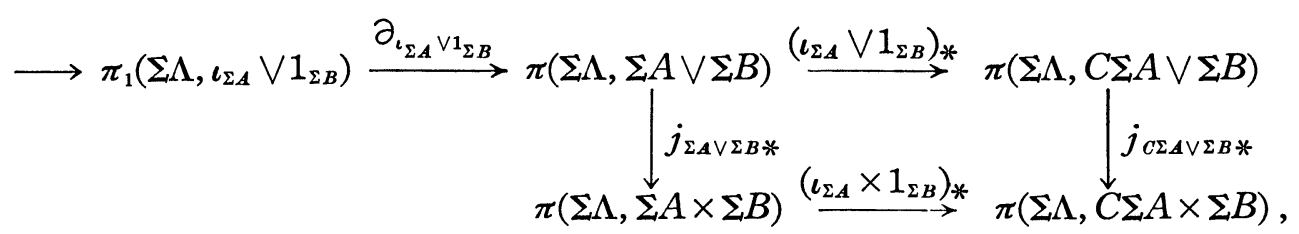

where the top row sequence is exact, and $j_{\Sigma \boldsymbol{A} \vee \Sigma B}: \Sigma A \vee \Sigma B \subset \Sigma A \times \Sigma B$, $j_{C \Sigma A \vee \Sigma B}: C \Sigma A \vee \Sigma B \subset C \Sigma A \times \Sigma B$. Then we have $j_{C \Sigma A \vee \Sigma B *} \circ\left(\iota_{\Sigma A} \vee 1_{\Sigma B}\right)_{*}(\theta)$ $=\left(\iota_{\Sigma A} \times 1_{\Sigma B}\right)_{*} j_{\Sigma A \vee \Sigma B *}(\theta)=0$ (c.f. [12]) and since $j_{C \Sigma A \vee \Sigma B *}$ is isomorphic we deduce that $\left(\iota_{\Sigma A} \vee 1_{\Sigma B}\right)_{*}=0$. So there is $\theta_{1} \in \pi_{1}\left(\Sigma \Lambda, \iota_{\Sigma A} \vee 1_{\Sigma B}\right)$ such that $\partial_{\iota_{\Sigma A} \vee 1_{\Sigma B}}\left(\theta_{1}\right)=\theta$. Since $\partial_{\iota_{\Sigma A} \vee 1_{\Sigma B}}$ is a monomorphism, the element $\theta_{1}$ is determined uniquely. Hence a representative of $\theta_{1}$ has the form $\left(\bar{\rho}_{A, B} \bar{h}_{A, B}, a\right)$ : $\iota \rightarrow \iota_{\Sigma A} \vee 1_{\Sigma B}$, where $\iota: \Sigma(A \# B) \rightarrow C \Sigma(A \# B)$, that is,

$$
\theta_{1}=\left\{\left(\bar{\rho}_{A, B} \bar{h}_{A, B}, a\right)\right\} \in \pi_{1}\left(\Sigma(A \# B), \iota_{\Sigma A} \vee 1_{\Sigma B}\right)
$$

Similarly we define 


$$
\theta_{1}^{\prime}=\left\{\left(\bar{\rho}_{B, A} \bar{h}_{B, A}, a^{\prime}\right)\right\} \in \pi_{1}\left(\Sigma(B \# A), 1_{\Sigma B} \bigvee \iota_{\Sigma A}\right)
$$

(c) Since $\partial_{\iota_{\Sigma A} \vee_{\iota_{\Sigma B}}}: \pi_{1}\left(\Sigma(A \# B), \iota_{\Sigma B} \vee \iota_{\Sigma B}\right) \cong \pi_{1}(\Sigma(A \# B), \Sigma A \vee \Sigma B)$ there exists $\theta_{2} \in \pi_{1}\left(\Sigma(A \# B), \iota_{\Sigma A} \vee \iota_{\Sigma B}\right)$ such that $\partial_{\iota_{\Sigma A} \iota_{\iota_{\Sigma B}}}\left(\theta_{2}\right)=\theta$.

On the other hand we have the commutative diagram

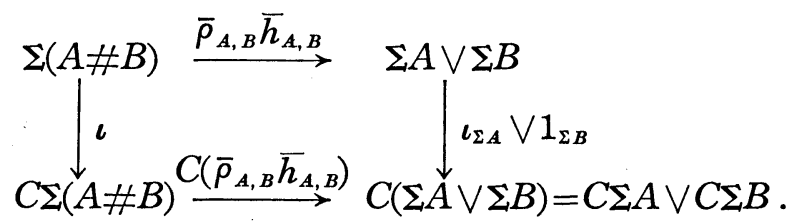

So a representative of $\theta_{2}$ is $\left(\bar{\rho}_{A, B} \bar{h}_{A, B}, C\left(\bar{\rho}_{A, B} \bar{h}_{A, B}\right)\right)$, that is,

$$
\theta_{2}=\left\{\left(\bar{\rho}_{A, B} \bar{h}_{A, B}, C\left(\bar{\rho}_{A, B} \bar{h}_{A, B}\right)\right\} \in \pi_{1}\left(\Sigma(A \# B), \iota_{\Sigma A} \vee \iota_{\Sigma B}\right)\right.
$$

Similarly we define

$$
\theta_{2}^{\prime}=\left\{\left(\bar{\rho}_{B, A} \bar{h}_{B, A}, C\left(\bar{\rho}_{B, A} \bar{h}_{B, A}\right)\right\} \in \pi_{1}\left(\Sigma(B \# A), \iota_{\Sigma B} \vee \iota_{\Sigma A}\right)\right.
$$

The homomorphism $\left(1_{\Sigma A} \bigvee 1_{\Sigma B}, 1_{C \Sigma B} \bigvee \iota_{\Sigma B}\right)_{*}: \pi_{1}\left(\Sigma(A \# B), \iota_{\Sigma A} \bigvee 1_{\Sigma B}\right) \rightarrow \pi_{1}(\Sigma(A \# B)$, $\left.\iota_{\Sigma A} \bigvee \iota_{\Sigma B}\right)$ induced by $\left(1_{\Sigma A} \vee 1_{\Sigma B}, 1_{C \Sigma A} \vee \iota_{\Sigma B}\right)$ is a monomorphism. Then we have

$$
\left(1_{\Sigma A} \bigvee 1_{\Sigma B}, 1_{C \Sigma A} \bigvee \iota_{\Sigma B}\right)_{*}\left(\theta_{1}\right)=\theta_{2}
$$

For, since $\theta=\partial_{\iota_{\Sigma A} \vee 1_{\Sigma B}}\left(\theta_{1}\right)=\partial_{\iota_{\Sigma A} \vee \iota_{\Sigma B}}\left(1_{\Sigma A} \vee 1_{\Sigma B}, 1_{C \Sigma A} \vee \iota_{\Sigma B}\right)_{*}\left(\theta_{1}\right)$ we have $\left(1_{\Sigma A} \vee 1_{\Sigma B}, 1_{C \Sigma A} \vee \iota_{\Sigma B}\right)_{*}\left(\theta_{1}\right)=\partial_{\iota_{\Sigma A} \vee \iota_{\Sigma B}}^{-1}(\theta)=\theta_{2}$

(d) In (2.1.1) we saw that $0=\left(1_{\Sigma A} \vee \iota_{\Sigma B}\right)_{*}(\theta)=\left(1_{\Sigma A} \vee \iota_{\Sigma B}\right)_{*} \circ \partial_{\iota_{\Sigma A} \vee 1_{\Sigma B}}\left(\theta_{1}\right)$ $=\partial_{\iota_{\Sigma A} \vee 1_{C \Sigma B}}\left(1_{\Sigma A} \vee \iota_{\Sigma B}, 1_{C \Sigma A} \vee \iota_{\Sigma B}\right)_{*}\left(\theta_{1}\right)$, and since $\partial_{\iota_{\Sigma A} \vee 1_{C \Sigma B}}$ is an isomorphism we have $\left(1_{\Sigma A} \bigvee \iota_{\Sigma B}, 1_{C \Sigma A} \bigvee \iota_{\Sigma B}\right)_{*}\left(\theta_{1}\right)=0$. Hence there is an element $\theta_{3} \in \pi_{2}(\Sigma(A \# B), \Psi)$ such that $\partial_{\psi}\left(\theta_{3}\right)=\theta_{1}$. Then since $\partial_{\Psi}$ is a monomorphism, $\theta_{3}$ is determined uniquely. And $\theta_{3}$ has a representative of the form $\left(\begin{array}{c}\bar{\rho}_{A, B} \bar{h}_{A, B} a \\ b\end{array}\right): \Pi \rightarrow \Psi$; that is,

$$
\theta_{3}=\left\{\left(\begin{array}{cc}
\bar{\rho}_{A, B} \bar{h}_{A, B} a \\
b & c
\end{array}\right)\right\} \in \pi_{2}(\Sigma(A \# B), \Psi),
$$

where $a: C \Sigma(A \# B) \rightarrow C \Sigma A \vee \Sigma B, \quad b: C \Sigma(A \# B) \rightarrow \Sigma A \vee C \Sigma B, c: C^{2} \Sigma(A \# B)$ $\longrightarrow C \Sigma A \vee C \Sigma B$ and $\Pi$ is the pair-map 


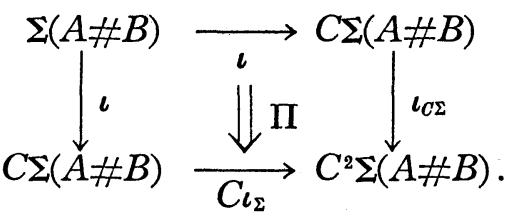

Similarly we define

$$
\theta_{3}^{\prime}=\left\{\left(\begin{array}{cr}
\bar{\rho}_{B, A} \bar{h}_{B, A} & a^{\prime} \\
b^{\prime} & c^{\prime}
\end{array}\right)\right\} \in \pi_{2}\left(\Sigma(B \# A), \Psi^{\prime}\right),
$$

where $\Psi^{\prime}=\left(\iota_{\Sigma B} \bigvee 1_{\Sigma A}, \iota_{\Sigma B} \bigvee 1_{C \Sigma A}\right): 1_{\Sigma B} \bigvee \iota_{\Sigma A} \rightarrow 1_{C \Sigma B} \bigvee \iota_{\Sigma A}$

(e) In (2.1.4), we had $0=\left(\iota_{\Sigma A} \vee 1_{\Sigma B}\right)_{*}(\theta)=\left(\iota_{\Sigma A} \vee 1_{\Sigma B}\right)_{*} \circ \partial_{\iota_{\Sigma A} \vee 1_{\Sigma B}}\left(\theta_{1}\right)$ $=\partial_{c_{\Sigma A} \vee 1_{\Sigma B}}\left(\iota_{\Sigma A} \vee 1_{\Sigma B}, 1_{C \Sigma A} \vee 1_{\Sigma B}\right)_{*}\left(\theta_{1}\right)$, and since $\partial_{c_{\iota_{\Sigma A}} \vee 1_{\Sigma B}}$ is an isomorphism we have $\left(\iota_{\Sigma A} \vee 1_{\Sigma B}, 1_{C \Sigma A} \bigvee 1_{\Sigma B}\right)_{*}\left(\theta_{1}\right)=0$. Hence there is an element $\theta_{4} \in \pi_{2}\left(\Sigma(A \# B), \Psi_{1}\right)$ such that $\partial_{\Psi_{1}}\left(\theta_{4}\right)=\theta_{1}$. And since $\partial_{\Psi_{1}}$ is a monomorphism $\theta_{4}$ is determined uniquely. Then the element $\theta_{4}$ has a representative of the form $\left(\begin{array}{c}\bar{\rho}_{A, B} \bar{h}_{A, B} a \\ \bar{b} \\ \bar{c}\end{array}\right)$ : $\Pi \rightarrow \Psi_{1}$; that is,

$$
\theta_{4}=\left\{\left(\begin{array}{cr}
\bar{\rho}_{A, B} \bar{h}_{A, B} a \\
\bar{b} & \bar{c}
\end{array}\right)\right\} \in \pi_{2}\left(\Sigma(A \# B), \Psi_{1}\right)
$$

where $\bar{b}: C \Sigma(A \# B) \rightarrow C \Sigma A \vee \Sigma B, \bar{c}: C^{2} \Sigma(A \# B) \rightarrow C^{2} \Sigma A \vee \Sigma B$

Similarly we define

$$
\theta_{4}^{\prime}=\left\{\left(\begin{array}{cc}
\bar{\rho}_{B, A} \bar{h}_{B, A} & a^{\prime} \\
\bar{b}^{\prime} & \bar{c}^{\prime}
\end{array}\right)\right\} \in \pi_{2}\left(\Sigma(B \# A), \Psi_{1}^{\prime}\right)
$$

where $\Psi_{1}^{\prime}=\left(1_{\Sigma B} \vee \iota_{\Sigma A}, 1_{\Sigma B} \vee \iota_{C \Sigma A}\right): 1_{\Sigma B} \vee \iota_{\Sigma A} \rightarrow 1_{\Sigma B} \vee C_{\iota_{\Sigma A}}$

(f) From (2.1.3) we see easily that $\theta_{2} \in \operatorname{Ker} .\left(\iota_{\Sigma A} \bigvee 1_{\Sigma B}, \iota_{C \Sigma A} \bigvee 1_{C \Sigma B}\right)_{*}$ $=\operatorname{Im} . \partial_{\Psi_{z}}$. Hence there is an element $\theta_{5} \in \pi_{2}\left(\Sigma(A \# B), \Psi_{2}\right)$ such that $\partial_{\Psi_{2}}\left(\theta_{5}\right)$ $=\theta_{2}$, and since $\partial_{\Psi_{1}}$ is a monomorphism $\theta_{5}$ is determined uniquely. Then the

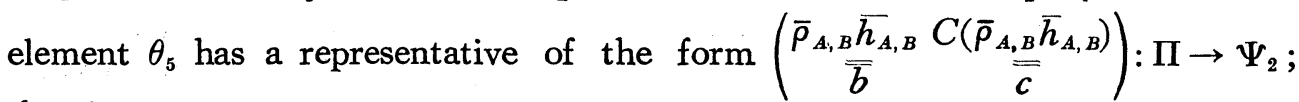
that is,

$$
\left.\theta_{5}=\left\{\left(\begin{array}{cc}
\bar{\rho}_{A, B} \bar{h}_{A, B} & C\left(\bar{\rho}_{A, B} \bar{h}_{A, B}\right) \\
\overline{\bar{b}} & \overline{\bar{c}}^{-}
\end{array}\right)\right\} \in \pi_{2} \Sigma(A \# B), \Psi_{2}\right),
$$


where $\bar{b}: C \Sigma(A \# B) \rightarrow C \Sigma A \vee \Sigma B, \overline{\bar{c}}: C^{2} \Sigma(A \# B) \rightarrow C^{2} \Sigma A \vee C \Sigma B$

Similarly we define

$$
\theta_{5}^{\prime}=\left\{\left(\bar{\rho}_{B, A} \bar{h}_{B, A} C\left(\bar{\rho}_{B, A} \bar{h}_{B, A}\right)\right)\right\} \in \pi_{2}\left(\Sigma(B \# A), \Psi_{2}^{\prime}\right),
$$

where $\Psi_{2}^{\prime}=\left(1_{\Sigma B} \bigvee \iota_{\Sigma A}, 1_{C \Sigma B} \bigvee \iota_{C \Sigma A}\right): \iota_{\Sigma B} \bigvee \iota_{\Sigma A} \rightarrow \iota_{\Sigma B} \bigvee C_{\iota_{\Sigma A}}$

(g) As we have seen in (2.1.2) $\partial_{\Psi_{3}}: \pi_{2}\left(\Sigma(A \# B), \Psi_{3}\right) \cong \pi_{1}\left(\Sigma(A \# B), \iota_{\Sigma A} \vee \iota_{\Sigma B}\right)$, so we obtain $\theta_{6} \in \pi_{2}\left(\Sigma(A \# B), \Psi_{3}\right)$ such that $\partial_{\Psi_{3}}\left(\theta_{6}\right)=\theta_{1}$, and $\theta_{6}$ is determined uniquely. And we have the $\operatorname{map}\left(\begin{array}{ll}\bar{\rho}_{A, B} \bar{h}_{A, B} & C\left(\bar{\rho}_{A, B} \bar{h}_{A, B}\right) \\ C\left(\bar{\rho}_{A, B} \bar{h}_{A, B}\right) & C^{2}\left(\bar{\rho}_{A, B} \bar{h}_{A, B}\right)\end{array}\right): \Pi \rightarrow \Psi_{3}$.

Hence a representative of $\theta_{B}$ is $\left(\begin{array}{ll}\bar{\rho}_{A, B} \bar{h}_{A, B} & C\left(\bar{\rho}_{A, B} \bar{h}_{A, B}\right) \\ C\left(\bar{\rho}_{A, B} \bar{h}_{A, B}\right) & C^{2}\left(\bar{\rho}_{A, B} \bar{h}_{A, B}\right)\end{array}\right)$, that is,

$$
\theta_{b}=\left\{\left(\begin{array}{ll}
\bar{\rho}_{A, B} \bar{h}_{A, B} & C\left(\bar{\rho}_{A, B} \bar{h}_{A, B}\right) \\
C\left(\bar{\rho}_{A, B} \bar{h}_{A, B}\right) & C^{2}\left(\bar{\rho}_{A, B} \bar{h}_{A, B}\right)
\end{array}\right)\right\} \in \pi_{2}\left(\Sigma(A \# B), \Psi_{3}\right) .
$$

Similarly we define

$$
\theta_{\theta}^{\prime}=\left\{\left(\begin{array}{ll}
\bar{\rho}_{B, A} \bar{h}_{B, A} & C\left(\bar{\rho}_{B, A} \bar{h}_{B, A}\right) \\
C\left(\bar{\rho}_{B, A} \bar{h}_{B, A}\right) & C^{2}\left(\bar{\rho}_{B, A} \bar{h}_{B, A}\right)
\end{array}\right)\right\} \in \pi_{2}\left(\Sigma(B \# A), \Psi_{3}^{\prime}\right),
$$

where $\Psi_{3}^{\prime}=\left(\iota_{\Sigma B} \bigvee \iota_{\Sigma A}, \iota_{C \Sigma B} \bigvee \iota_{C \Sigma A}\right): \iota_{\Sigma B} \bigvee \iota_{\Sigma A} \rightarrow C_{\iota_{\Sigma B}} \vee C_{\iota_{\Sigma A}}$

Let $\quad \varphi_{*}: \pi_{2}(\Sigma(A \# B), \Psi) \rightarrow \pi_{2}\left(\Sigma(A \# B), \Psi_{3}\right), \quad \varphi_{2,3 *}: \quad \pi_{2}\left(\Sigma(A \# B), \Psi_{2}\right) \rightarrow$ $\pi_{2}\left(\Sigma(A \# B), \Psi_{3}\right)$ and $\varphi_{1,2 *}: \quad \pi_{2}\left(\Sigma(A \# B), \Psi_{1}\right) \rightarrow \pi_{2}\left(\Sigma(A \# B), \Psi_{2}\right)$ be natural homomorphisms induced by $\boldsymbol{\phi}, \boldsymbol{\phi}_{2,3}$ and $\boldsymbol{\varphi}_{1,2}$, respectively, where $\boldsymbol{\phi}$ $=\left(\begin{array}{ll}1_{\Sigma A} \bigvee 1_{\Sigma B} & 1_{C \Sigma A} \bigvee \iota_{\Sigma B} \\ \iota_{\Sigma A} \vee 1_{C \Sigma B} & \iota_{C \Sigma A} \vee \iota_{\Sigma B}\end{array}\right): \Psi \rightarrow \Psi_{3}, \varphi_{2,3}=\left(\begin{array}{ll}1_{\Sigma A} \bigvee 1_{\Sigma B} & 1_{C \Sigma A} \vee 1_{C \Sigma B} \\ 1_{C \Sigma A} \bigvee \iota_{C \Sigma B} & 1_{C \Sigma \Sigma A} \bigvee \iota_{C \Sigma B}\end{array}\right): \Psi_{2} \rightarrow \Psi_{3}$, and $\phi_{1,2}=\left(\begin{array}{ll}1_{\Sigma A} \bigvee 1_{\Sigma B} & 1_{C \Sigma A} \bigvee \iota_{\Sigma B} \\ 1_{C \Sigma A} \bigvee 1_{\Sigma B} & 1_{C^{2} \Sigma A} \bigvee \iota_{\Sigma B}\end{array}\right): \Psi_{1} \rightarrow \Psi_{2} . \quad$ Then we see easily

$$
\varphi_{*}\left(\theta_{3}\right)=\boldsymbol{\varphi}_{2,3 *}\left(\theta_{5}\right)=\theta_{6} \text { and } \boldsymbol{\varphi}_{1,2 *}\left(\theta_{4}\right)=\theta_{5}
$$

3. The generalized Whitehead products. By using the generelized Whitehead elements defined in section 2 , we may define the various products in the generalized homotopy groups.

(A) The generalized Whitehead product (GWP) $[\alpha, \beta] \in \pi(\Sigma(A \# B), X)$ of $\{f\}=\alpha \in \pi(\Sigma A, X)$ and $\{g\}=\beta \in \pi(\Sigma B, X)$ is defined as follows [2], [12] : 


$$
[\alpha, \beta]=(\alpha, \beta)_{*}(\theta) \text {, where }(\alpha, \beta)=\nabla \circ(f \vee g) \text {, }
$$

that is,

$$
[\alpha, \beta]=\left\{\nabla \circ(f \vee g) \circ \bar{\rho}_{A, B} \bar{h}_{A, B}\right\} \in \pi(\Sigma(A \# B), X) .
$$

(B) The GWP $[\alpha, \beta]_{1} \in \pi_{1}(\Sigma(A \# B), k)$ of $\left\{\left(f, f^{\prime}\right)\right\}=\alpha \in \pi_{1}(\Sigma A, k)$ and $\{g\}=\beta \in \pi(\Sigma B, X), k: X \rightarrow Y$, is defined by

$$
[\alpha, \beta]_{1}=(\alpha, \beta)_{1^{*}}\left(\theta_{1}\right), \text { where }(\alpha, \beta)_{1}=\left(\nabla \circ(f \vee g), \nabla \circ\left(f^{\prime} \vee k g\right)\right),
$$

that is,

$$
[\alpha, \beta]_{1}=\left\{\left(\nabla \circ(f \vee g) \circ \bar{\rho}_{A, B} \bar{h}_{A, B}, \nabla \circ\left(f^{\prime} \vee k y\right) \circ a\right)\right\} \in \pi_{1}(\Sigma(A \# B), k) .
$$

Then $[\alpha, \beta]_{1}$ is independent of choice of $\left(f, f^{\prime}\right)$ and $g$. Similarly we define

$$
[\beta, \alpha]_{1}=(\beta, \alpha)_{1 *}\left(\theta_{1}^{\prime}\right) \text {, where }(\beta, \alpha)_{1}=\left(\nabla \circ(g \vee f), \nabla \circ\left(k g \vee f^{\prime}\right)\right),
$$

that is,

$$
[\beta, \alpha]_{1}=\left\{\left(\nabla \circ(g \vee f) \circ \bar{\rho}_{B, A} \bar{h}_{B, A}, \nabla \circ\left(k g \vee f^{\prime}\right) \circ a^{\prime}\right)\right\} \in \pi_{1}(\Sigma(B \# A), k) .
$$

REMARK. This GWP is a generalization of the relative product in the sense of M. G. Baratt [9; p. 164].

(C) The GWP $[\alpha, \beta]_{2} \in \pi_{1}(\Sigma(A \# B), k)$ of $\left\{\left(f, f^{\prime}\right)\right\}=\alpha \in \pi_{1}(\Sigma A, k)$ and $\left\{\left(g, g^{\prime}\right)\right\}=\beta \in \pi_{1}(\Sigma B, k), k: X \rightarrow Y$, is defined by

$$
[\alpha, \beta]_{2}=(\alpha, \beta)_{2 *}\left(\theta_{2}\right), \text { where }(\alpha, \beta)_{2}=\left(\nabla \circ(f \vee g), \nabla \circ\left(f^{\prime} \vee g^{\prime}\right)\right),
$$

that is,

$$
[\alpha, \beta]_{2}=\left\{\left(\nabla \circ(f \vee g) \circ \bar{\rho}_{A, B} \bar{h}_{A, B}, \nabla \circ\left(f^{\prime} \vee g^{\prime}\right) \circ C\left(\bar{\rho}_{A, B} \bar{h}_{A, B}\right)\right\} \in \pi_{1}(\Sigma(A \# B), k) .\right.
$$

Then $[\alpha, \beta]_{2}$ is independent of the choice of $\left(f, f^{\prime}\right)$ and $\left(g, g^{\prime}\right)$.

Similarly we define

$$
[\beta, \alpha]_{2}=(\beta, \alpha)_{2 *}\left(\theta_{2}^{\prime}\right), \text { where }(\beta, \alpha)_{2}=\left(\nabla \circ(g \vee f), \nabla \circ\left(g^{\prime} \vee f^{\prime}\right)\right),
$$

that is,

$$
\left.[\beta, \alpha]_{2}=\left\{\left(\nabla \circ(g \vee f) \circ \bar{\rho}_{A, B} \bar{h}_{B, A}\right), \nabla \circ\left(g^{\prime} \vee f^{\prime}\right) \circ C\left(\bar{\rho}_{B, A} \bar{h}_{B_{1} A}\right)\right)\right\} \in \pi_{1}(\Sigma(B \# A), k) .
$$


526

H. AND

(D) We consider a pair-map $\Phi=\left(v, v^{\ell}\right): u \rightarrow u^{\prime}$;

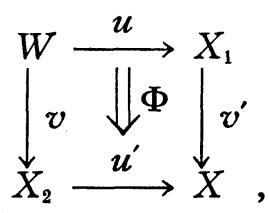

Hereafter, in this paper $\Phi$ denotes the above map. Then the GWP $[\alpha, \beta]_{3} \in \pi_{2}(\Sigma(A \# B), \Phi)$ of $\left\{\left(f, f^{\prime}\right)\right\}=\alpha \in \pi_{1}(\Sigma A, u)$ and $\left\{\left(g, g^{\prime}\right)\right\}=\beta \in \pi_{1}(\Sigma B, v)$ is given by

(3.4) $[\alpha, \beta]_{3}=(\alpha, \beta)_{3 *}(\theta)_{3}$, where $(\alpha, \beta)_{3}=\left(\begin{array}{ll}\nabla \circ(f \vee g) & \nabla \circ\left(f^{\prime} \vee u g\right) \\ \nabla \circ\left(v f \vee g^{\prime}\right) & \nabla \circ\left(v^{\prime} f \vee u^{\prime} g^{\prime}\right)\end{array}\right)$, that is,

$$
[\alpha, \beta]_{3}=\left\{\left(\begin{array}{ll}
\nabla \circ(f \vee g) \circ \bar{\rho}_{A, B} \bar{h}_{A, B} & \nabla \circ\left(f^{\prime} \vee u g\right) \circ a \\
\nabla \circ\left(v f \vee g^{\prime}\right) \circ b & \nabla \circ\left(v^{\prime} f \vee u^{\prime} g^{\prime}\right) \circ c
\end{array}\right)\right\} \in \pi_{2}(\Sigma(A \# B), \Phi) .
$$

Then $[\alpha, \beta]_{3}$ is independent of the choice of $\left(f, f^{\prime}\right)$ and $\left(g, g^{\prime}\right)$.

Similarly we define

(3. $\left.4^{\prime}\right) \quad[\beta, \alpha]_{3}=(\beta, \alpha)_{3 *}\left(\theta_{3}^{\prime}\right)$, where $(\beta, \alpha)_{3}=\left(\begin{array}{ll}\nabla \circ(g \vee f) & \nabla \circ\left(u g \vee f^{\prime}\right) \\ \nabla \circ\left(g^{\prime} \bigvee v f\right) & \nabla \circ\left(u^{\prime} g^{\prime} \vee v^{\prime} f\right)\end{array}\right)$, that is,

$$
[\beta, \alpha]_{3}=\left\{\left(\begin{array}{ll}
\nabla \circ(g \bigvee f) \circ \bar{\rho}_{A, B} \bar{h}_{A, B} & \nabla \circ\left(u g \bigvee f^{\prime}\right) \circ a^{\prime} \\
\nabla \circ\left(g^{\prime} \bigvee v f\right) \circ b^{\prime} & \nabla \circ\left(u^{\prime} y^{\prime} \vee v^{\prime} f\right) \circ c^{\prime}
\end{array}\right)\right\} \in \pi_{2}(\Sigma(B \# A), \Phi) .
$$

(E) The GWP $[\alpha, \beta]_{4} \in \pi_{2}(\Sigma(A \# B), \Phi)$ of $\left\{\left(\begin{array}{ll}f & f^{\prime} \\ f^{\prime \prime} & f^{\prime \prime \prime}\end{array}\right)\right\}=\alpha \in \pi_{2}(\Sigma A, \Phi)$ and $\{g\}=\boldsymbol{\beta} \in \pi(\Sigma B, W)$ is defined by

(3. 5) $[\alpha, \beta]_{4}=(\alpha, \beta)_{4 *}\left(\theta_{4}\right)$, where $(\alpha, \beta)_{4}=\left(\begin{array}{ll}\nabla \circ(f \vee g) & \nabla \circ\left(f^{\prime} \vee u g\right) \\ \nabla \circ\left(f^{\prime \prime} \vee v g\right) & \nabla \circ\left(f^{\prime \prime \prime} \vee v^{\prime} u g\right)\end{array}\right)$,

that is,

$$
[\alpha, \beta]_{4}=\left\{\begin{array}{ll}
\nabla \circ(f \vee g) \circ \bar{\rho}_{A, B} \bar{h}_{A, B} & \nabla \circ\left(f^{\prime} \vee u g\right) \circ \bar{a} \\
\nabla \circ\left(f^{\prime \prime} \vee v g\right) \circ \bar{b} & \nabla \circ\left(f^{\prime \prime \prime} \vee v^{\prime} u g\right) \circ \bar{c}
\end{array}\right) \in \pi_{2}(\Sigma(A \# B), \Phi) .
$$

Then $[\alpha, \beta]_{4}$ is independent of the choice of $\left(\begin{array}{l}f f^{\prime} \\ f^{\prime \prime} f^{\prime \prime \prime}\end{array}\right)$ and $g$. 
Similarly we define

$$
[\beta, \alpha]_{4}=(\beta, \alpha)_{4 *}\left(\theta_{4}^{\prime}\right), \text { where }(\beta, \alpha)_{4}=\left(\begin{array}{ll}
\nabla \circ(g \vee f) & \nabla \circ\left(u g \vee f^{\prime}\right) \\
\nabla \circ\left(v g \vee f^{\prime \prime}\right) & \nabla \circ\left(v^{\prime} u g \vee f^{\prime \prime \prime}\right)
\end{array}\right),
$$

that is,

$$
[\beta, \alpha]_{4}=\left\{\begin{array}{ll}
\nabla \circ(g \vee f) \circ \bar{\rho}_{B, A} \bar{h}_{B, 4} & \nabla \circ\left(u g \vee f^{\prime}\right) \circ \bar{a}^{\prime} \\
\nabla \circ\left(v g \vee f^{\prime \prime}\right) \circ \bar{b}^{\prime} & \nabla \circ\left(v^{\prime} u g \vee f^{\prime \prime \prime}\right) \circ \bar{c}^{\prime}
\end{array}\right) \in \pi_{2}(\Sigma(B \# A), \Phi) .
$$

(F) The GWP $[\alpha, \beta]_{5} \in \pi_{2}(\Sigma(A \# B), \Phi)$ of $\left\{\left(\begin{array}{ll}f & f^{\prime} \\ f^{\prime \prime} & f^{\prime \prime \prime}\end{array}\right)\right\}=\alpha \in \pi_{2}(\Sigma A, \Phi)$ and $\left\{\left(g, g^{\prime}\right)\right\}=\beta \in \pi_{1}(\Sigma B, u)$ is defined by

(3. 6) $[\alpha, \beta]_{5}=(\alpha, \beta)_{5 *}\left(\theta_{5}\right)$, where $(\alpha, \beta)_{5}=\left(\begin{array}{ll}\nabla \circ(f \vee g) & \nabla \circ\left(f^{\prime} \vee g^{\prime}\right) \\ \nabla \circ\left(f^{\prime \prime} \vee v g\right) & \nabla \circ\left(f^{\prime \prime \prime} \vee v^{\prime} g^{\prime}\right)\end{array}\right)$, that is,

$$
[\alpha, \beta]_{5}=\left\{\left(\begin{array}{ll}
\nabla \circ(f \vee g) \circ \bar{\rho}_{A, B} \bar{h}_{A, B} & \nabla \circ\left(f^{\prime} \vee g^{\prime}\right) \circ C\left(\bar{\rho}_{A, B} \bar{h}_{A, B}\right) \\
\nabla \circ\left(f^{\prime \prime} \vee v g\right) \circ \bar{b} & \nabla \circ\left(f^{\prime \prime \prime} \vee v^{\prime} g^{\prime}\right) \circ \overline{\bar{c}}
\end{array}\right)\right\} \in \pi_{2}(\Sigma(A \# B), \Phi) .
$$

Then $[\alpha, \beta]_{5}$ is independent of the choice of $\left(\begin{array}{ll}f & f^{\prime} \\ f^{\prime \prime} & f^{\prime \prime \prime}\end{array}\right)$ and $\left(g, g^{\prime}\right)$.

Similarly we define

(3. $\left.6^{\prime}\right)[\beta, \alpha]_{5}=(\beta, \alpha)_{5 *}\left(\theta_{5}^{\prime}\right)$, where $(\beta, \alpha)_{5}=\left(\begin{array}{ll}\nabla \circ(g \vee f) & \nabla \circ\left(g^{\prime} \vee f^{\prime}\right) \\ \nabla \circ\left(v g \vee f^{\prime \prime}\right) & \nabla \circ\left(v^{\prime} g^{\prime} \vee f^{\prime \prime \prime}\right)\end{array}\right)$,

that is,

$$
[\beta, \alpha]_{5}=\left\{\left(\begin{array}{ll}
\nabla \circ(g \vee f) \circ \bar{\rho}_{B, A} \bar{h}_{B, A} & \nabla \circ\left(g^{\prime} \vee f^{\prime}\right) \circ C\left(\bar{\rho}_{B, A} \bar{h}_{B, A}\right) \\
\nabla \circ\left(v g \vee f^{\prime \prime}\right) \circ \bar{b}^{\prime} & \nabla \circ\left(v^{\prime} g^{\prime} \vee f^{\prime \prime \prime}\right) \circ \bar{c}^{\prime}
\end{array}\right\} \in \pi_{2}(\Sigma(B \# A), \Phi),\right.
$$

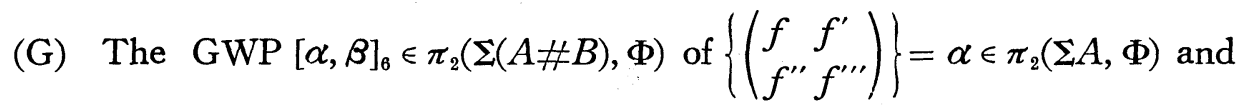
$\left\{\left(\begin{array}{cc}g & g^{\prime} \\ g^{\prime \prime} & g^{\prime \prime \prime}\end{array}\right)\right\}=\beta \in \pi_{2}(\Sigma B, \Phi)$ is defined by

(3. 7) $[\alpha, \beta]_{6}=(\alpha, \beta)_{6 *}\left(\theta_{6}\right)$, where $(\alpha, \beta)_{6}=\left(\begin{array}{ll}\nabla \circ(f \vee g) & \nabla \circ\left(f^{\prime} \vee g^{\prime}\right) \\ \nabla \circ\left(f^{\prime \prime} \vee g^{\prime \prime}\right) & \nabla \circ\left(f^{\prime \prime \prime} \vee g^{\prime \prime \prime}\right)\end{array}\right)$, that is, 


$$
\begin{aligned}
{[\alpha, \beta]_{6} } & =\left\{\left(\begin{array}{ll}
\nabla \circ(f \vee g) \circ \bar{\rho}_{A, B} \bar{h}_{A, B} & \nabla \circ\left(f^{\prime} \vee g^{\prime}\right) \circ C\left(\bar{\rho}_{A, B} \bar{h}_{A, B}\right) \\
\nabla \circ\left(f^{\prime \prime} \vee g^{\prime \prime}\right) \circ C\left(\bar{\rho}_{A, B} \bar{h}_{A, B}\right) & \nabla \circ\left(f^{\prime \prime \prime} \vee g^{\prime \prime \prime}\right) \circ C^{2}\left(\bar{\rho}_{A, B} \bar{h}_{A, B}\right)
\end{array}\right)\right\} \\
& \in \pi_{2}(\Sigma(A \# B), \Phi)
\end{aligned}
$$

Then $[\alpha, \beta]_{6}$ is independent of the choice of $\left(\begin{array}{ll}f & f^{\prime} \\ f^{\prime \prime} & f^{\prime \prime \prime}\end{array}\right)$ and $\left(\begin{array}{ll}g & g^{\prime} \\ g^{\prime \prime} & g^{\prime \prime \prime}\end{array}\right)$.

Similarly we define

(3. $\left.7^{\prime}\right)[\beta, \alpha]_{6}=(\beta, \alpha)_{6 *}\left(\theta_{6}^{\prime}\right)$, where $(\beta, \alpha)_{6}=\left(\begin{array}{ll}\nabla \circ(g \vee f) & \nabla \circ\left(g^{\prime} \vee f^{\prime}\right) \\ \nabla \circ\left(g^{\prime \prime} \vee f^{\prime \prime}\right) & \nabla \circ\left(g^{\prime \prime \prime} \vee f^{\prime \prime \prime}\right)\end{array}\right)$,

that is,

$$
\begin{aligned}
{[\beta, \alpha]_{6} } & =\left\{\left(\begin{array}{ll}
\nabla \circ(g \vee f) \circ \bar{\rho}_{B, A} \bar{h}_{B, A} & \nabla \circ\left(g^{\prime} \vee f^{\prime}\right) \circ C\left(\bar{\rho}_{B, A} \bar{h}_{B, A}\right) \\
\nabla \circ\left(g^{\prime \prime} \vee f^{\prime \prime}\right) \circ C\left(\bar{\rho}_{B, A} \bar{h}_{B, A}\right) & \nabla \circ\left(g^{\prime \prime \prime} \vee f^{\prime \prime \prime}\right) \circ C\left(\bar{\rho}_{B, A} \bar{h}_{B, A}\right)
\end{array}\right)\right\} \\
& \in \pi_{2}(\Sigma(B \# A), \Phi) .
\end{aligned}
$$

4. Properties of the generalized Whitehead products. We consider properties of the generalized Whitehead products defined in section 3 .

Let $k: X \rightarrow Y$ be a map and let $(m, n): k \rightarrow k^{\prime}$ be a pair-map :

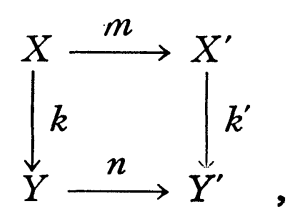

and let $\left(\begin{array}{ll}a_{1} & a_{2} \\ b_{1} & b_{2}\end{array}\right): \Phi \rightarrow \bar{\Phi}$ be a map

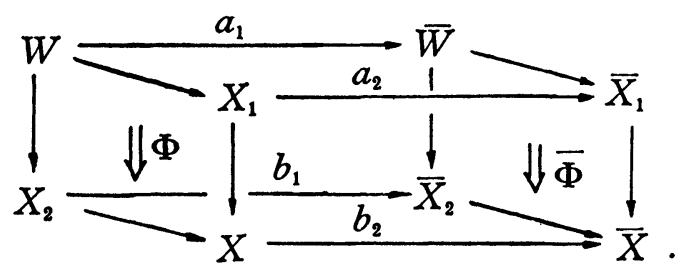

Then we obtain easily the following formulas :

PROPOSITION (4.1)

$$
k_{*}[\alpha, \beta]=\left[k_{*} \alpha, k_{*} \beta\right] \quad \text { for } \alpha \in \pi(\Sigma A, X), \beta \in \pi(\Sigma B, X),
$$


$(m, n)_{*}[\alpha, \beta]_{1}=\left[(m, n)_{*} \alpha, m_{*} \beta\right]_{1}$

$(m, n)_{*}[\alpha, \beta]_{2}=\left[(m, n)_{*} \alpha,(m, n)_{*} \beta\right]_{2} \quad$ for $\alpha \in \pi_{1}(\Sigma A, k), \beta \in \pi_{1}(\Sigma B, k)$,

$\left(\begin{array}{ll}a_{1} & a_{2} \\ b_{1} & b_{2}\end{array}\right)_{*}[\alpha, \beta]_{3}=\left[\left(a_{1}, a_{2}\right)_{*} \alpha,\left(a_{1}, b_{1}\right)_{*} \beta\right]_{3} \quad$ for $\alpha \in \pi_{1}(\Sigma A, u), \beta \in \pi_{1}(\Sigma B, v)$,

$\left(\begin{array}{ll}a_{1} & a_{2} \\ b_{1} & b_{2}\end{array}\right)_{*}[\alpha, \beta]_{4}=\left[\left(\begin{array}{ll}a_{1} & a_{2} \\ b_{1} & b_{2}\end{array}\right)_{*} \alpha, a_{1 *} \beta\right]_{4} \quad$ for $\alpha \in \pi_{2}(\Sigma A, \Phi), \beta \in(\Sigma B, W)$,

$\left(\begin{array}{ll}a_{1} & a_{2} \\ b_{1} & b_{2}\end{array}\right)_{*}[\alpha, \beta]_{5}=\left[\left(\begin{array}{ll}a_{1} & a_{2} \\ b_{1} & b_{2}\end{array}\right)_{*} \alpha,\left(a_{1}, a_{2}\right)_{*} \beta\right]_{5}$ for $\alpha \in \pi_{2}(\Sigma A, \Phi), \beta \in(\Sigma B, u)$,

$\left(\begin{array}{ll}a_{1} & a_{2} \\ b_{1} & b_{2}\end{array}\right)_{*}[\alpha, \beta]_{6}=\left[\left(\begin{array}{ll}a_{1} & a_{2} \\ b_{1} & b_{2}\end{array}\right)_{*} \alpha,\left(\begin{array}{ll}a_{1} & a_{2} \\ b_{1} & b_{2}\end{array}\right)_{*} \beta\right]_{6}$ for $\alpha \in \pi_{2}(\Sigma A, \Phi), \beta \in \pi_{2}(\Sigma B, \Phi)$.

Let $\partial_{k}: \pi_{1}(\Sigma D, k) \longrightarrow \pi(\Sigma D, X), \quad \partial_{k}: \pi_{1}(\Sigma \Lambda, k) \longrightarrow \pi(\Sigma \Lambda, X)$,

$$
\partial_{v}: \pi_{1}(\Sigma B, v) \longrightarrow \pi(\Sigma B, W), \quad \partial_{\Phi}: \pi_{2}(\Sigma \Lambda, \Phi) \longrightarrow \pi_{1}(\Sigma \Lambda, u)
$$

denote boundary homomorphisms, where $D=A$ or $B$ and $\Sigma \Lambda=\Sigma(A \# B)$ or $\Sigma(B \# A)$.

Then we have

\section{PROPOSITION (4.2)}

$\partial_{k}[\alpha, \beta]_{1}=\left[\partial_{k} \alpha, \beta\right], \partial_{k}[\beta, \alpha]_{1}=\left[\beta, \partial_{k} \alpha\right] \quad$ for $\alpha \in \pi_{1}(\Sigma A, k), \beta \in \pi(\Sigma B, X)$, $\partial_{k}[\alpha, \beta]_{2}=\left[\partial_{k} \alpha, \partial_{k} \beta\right], \partial_{k}[\beta, \alpha]_{2}=\left[\partial_{k} \beta, \partial_{k} \alpha\right]$ for $\alpha \in \pi_{1}(\Sigma A, k), \beta \in \pi_{1}(\Sigma B, k)$, $\partial_{\Phi}[\alpha, \beta]_{3}=\left[\alpha, \partial_{v} \beta\right]_{1}, \partial_{\Phi}[\beta, \alpha]_{3}=\left[\partial_{v} \beta, \alpha\right]_{1} \quad$ for $\alpha \in \pi_{1}(\Sigma A, u), \beta \in \pi_{1}(\Sigma B, v)$, $\partial_{\Phi}[\alpha, \beta]_{4}=\left[\partial_{\Psi} \alpha, \beta\right]_{1}, \partial_{\Phi}[\beta, \alpha]_{4}=\left[\beta, \partial_{\Phi} \alpha\right]_{1} \quad$ for $\alpha \in \pi_{2}(\Sigma A, \Phi), \beta \in \pi(\Sigma B, W)$, $\partial_{\Phi}[\alpha, \beta]_{5}=\left[\partial_{\Phi} \alpha, \beta\right]_{2}, \partial_{\Phi}[\beta, \alpha]_{5}=\left[\beta, \partial_{\Phi} \alpha\right]_{2} \quad$ for $\alpha \in \pi_{2}(\Sigma A, \Phi), \beta \in \pi_{1}(\Sigma B, u)$, $\partial_{\Phi}[\alpha, \beta]_{6}=\left[\partial_{\Phi} \alpha, \partial_{\Phi} \beta\right]_{2}, \partial_{\Phi}[\beta, \alpha]_{6}=\left[\partial_{\Phi} \beta, \partial_{\Phi} \alpha\right]_{2}$ for $\alpha \in \pi_{2}(\Sigma A, \Phi), \beta \in \pi_{2}(\Sigma B, \Phi)$.

Proof. We shall prove $\partial_{k}[\alpha, \beta]_{1}=\left[\partial_{k} \alpha, \beta\right]$. Proofs of the other five formulas are similar to it. We consider the following commutative diagram

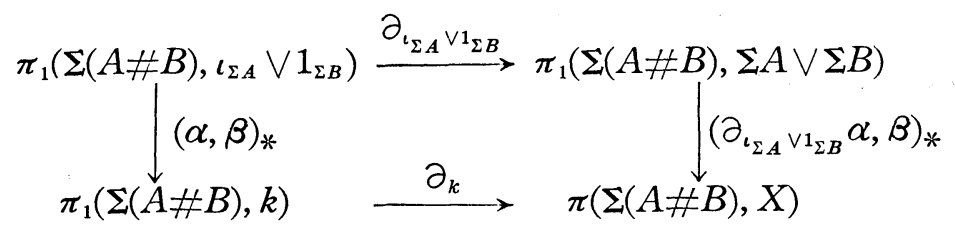

Then we have $\partial_{k}[\alpha, \beta]_{1}=\partial_{k}(\alpha, \beta)_{1 *}\left(\theta_{1}\right)=\left(\partial_{k} \alpha, \beta\right)_{*} \circ \partial_{\iota_{\Sigma A} \vee 1_{\Sigma \beta}}\left(\theta_{1}\right)=\left(\partial_{k} \alpha, \beta\right)_{*}(\theta)$ $=\left[\partial_{k} \alpha, \beta\right]$. 
We have the following properties for the relations of GWP's defined in section 3 .

PROPOSITION (4.3)

$$
\begin{aligned}
& {[\alpha, \beta]_{2}=\left[\alpha, \partial_{k} \beta\right]_{1},} \\
& {[\beta, \alpha]_{2}=\left[\partial_{k} \beta, \alpha\right]_{1},} \\
& \text { for } \alpha \in \pi_{1}(\Sigma A, k), \beta \in \pi_{1}(\Sigma B, k) \text {, } \\
& {[\alpha, \beta]_{5}=\left[\alpha, \partial_{u} \beta\right]_{4} \text {, }} \\
& {[\beta, \alpha]_{5}=\left[\partial_{u} \beta, \alpha\right]_{4},} \\
& \text { for } \alpha \in \pi_{2}(\Sigma A, \Phi), \beta \in \pi_{1}(\Sigma B, u) \text {, } \\
& {[\alpha, \beta]_{\mathrm{B}}=\left[\partial_{\Phi} \alpha, \partial_{\Phi}{ }^{T \circ \tau}(\beta)\right]_{3},} \\
& {[\beta, \alpha]_{B}=\left[\partial_{\Phi}{ }^{T \circ \tau}(\beta), \partial_{\Phi} \alpha\right]_{3},} \\
& \text { for } \alpha \in \pi_{2}(\Sigma A, \Phi), \beta \in \pi_{2}(\Sigma B, \Phi) \text {, } \\
& {[\alpha, \beta]_{6}=\left[\alpha, \partial_{4} \beta\right]_{5} \text {, }} \\
& {[\beta, \alpha]_{6}=\left[\partial_{\Phi} \beta, \alpha\right]_{5},} \\
& \text { for } \alpha \in \pi_{2}(\Sigma A, \Phi), \beta \in \pi_{2}(\Sigma B, \Phi) \text {, }
\end{aligned}
$$

where $\tau: \pi_{2}(\Sigma B, \Phi) \cong \pi_{2}\left(\Sigma B, \Phi^{T}\right)$ is the natural isomorphism defined in [5; p. 291] and $\partial_{\Phi^{T}}: \pi_{2}\left(\Sigma B, \Phi^{T}\right) \rightarrow \pi_{1}(\Sigma B, v)$ is the boundary homomorphism.

ProOF. To prove $[\alpha, \beta]_{2}=\left[\alpha, \partial_{k} \beta\right]_{1}$, let $\alpha=\left\{\left(f, f^{\prime}\right)\right\}$ and $\beta=\left\{\left(g, g^{\prime}\right)\right\}$.

By (2.6) we have $(\alpha, \beta)_{2 *}\left(\theta_{2}\right)=(\alpha, \beta)_{2 *}\left(1_{\Sigma A} \vee 1_{\Sigma B}, 1_{C \Sigma A} \vee \iota_{\Sigma B}\right)_{*}\left(\theta_{1}\right)$, where $(\alpha, \beta)_{2}=\left(\nabla \circ(f \vee g), \nabla \circ\left(f^{\prime} \vee g^{\prime}\right)\right)$. Hence we obtain

$$
\begin{aligned}
(\alpha, \beta)_{2} \circ\left(\rho_{A, B} \bar{h}_{A, B}, C\left(\bar{\rho}_{A, B} \bar{h}_{A, B}\right)\right) & \simeq(\alpha, \beta)_{2}\left(1_{\Sigma A} \vee 1_{\Sigma B}, 1_{C \Sigma A} \vee \iota_{\Sigma B}\right)\left(\bar{\rho}_{A, B} \bar{h}_{A, B}, a\right) \\
& =\left(\nabla \circ(f \vee g) \circ \bar{\rho}_{A, B} \bar{h}_{A, B}, \nabla \circ\left(f^{\prime} \vee k g\right) \circ a\right) \\
& =\left(\alpha, \partial_{k} \beta\right)_{1} \circ\left(\bar{\rho}_{A, B} \bar{h}_{A, B}, a\right) .
\end{aligned}
$$

Thus $[\alpha, \beta]_{2}=(\alpha, \beta)_{2 *}\left(\theta_{2}\right)=\left(\alpha, \partial_{k} \beta\right)_{1 *}\left(\theta_{1}\right)=\left[\alpha, \partial_{k} \beta\right]_{1}$. By using the formula analogous to $(2.6)$ we have similarly $[\beta, \alpha]_{2}=\left[\partial_{k} \beta, \alpha\right]_{1}$.

Next we shall prove that $[\alpha, \beta]_{6}=\left[\partial_{\Phi} \alpha, \partial_{\Phi^{T}} \circ \tau(\beta)\right]_{3}$. Let $\alpha=\left\{\left(\begin{array}{ll}f & f^{\prime} \\ f^{\prime \prime} & f^{\prime \prime \prime}\end{array}\right)\right\}$ and $\beta=\left\{\left(\begin{array}{ll}g & g^{\prime} \\ g^{\prime \prime} & g^{\prime \prime \prime}\end{array}\right)\right\}$, then $\left(\begin{array}{ll}g & g^{\prime} \\ g^{\prime} & g^{\prime \prime \prime} u\end{array}\right),\left(g, g^{\prime \prime}\right)$ and $\left(f, f^{\prime}\right)$ are representatives of $\tau(\beta), \partial_{\Phi^{T} \circ \tau}(\beta)$ and $\partial_{\Phi}(\alpha)$, respectively, where $u: C^{2} B \rightarrow C^{2} B$ is the homeomorphism defined by $u(b, s, t)=(b, t, s)[5]$. By $(2.11)(\alpha, \beta)_{6 *} \boldsymbol{\varphi}_{*}\left(\theta_{3}\right)=(\alpha, \beta)_{6 *}\left(\theta_{6}\right)$. Hence we obtain 


$$
\begin{aligned}
(\alpha, \beta)_{6} \circ\left(\begin{array}{ll}
\bar{\rho}_{A, B} \bar{h}_{A, B} & C\left(\bar{\rho}_{A, B} \bar{h}_{A, B}\right) \\
C\left(\bar{\rho}_{A, B} \bar{h}_{A, B}\right) & C^{2}\left(\bar{\rho}_{A, B} \bar{h}_{A, B}\right)
\end{array}\right) \simeq(\alpha, \beta)_{6} \circ \varphi\left(\begin{array}{cc}
\rho_{A, B} h_{A, B} & a \\
b & c
\end{array}\right) \\
=\left(\begin{array}{ll}
\nabla \circ(f \vee g) & \nabla \circ\left(f^{\prime} \vee g^{\prime}\right) \\
\nabla \circ\left(f^{\prime \prime} \vee g^{\prime \prime}\right) & \nabla \circ\left(f^{\prime \prime \prime} \vee g^{\prime \prime \prime}\right)
\end{array}\right) \circ\left(\begin{array}{cc}
1_{\Sigma A} \vee 1_{\Sigma B} & 1_{C \Sigma A} \vee \iota_{\Sigma B} \\
\iota_{\Sigma A} \vee 1_{C \Sigma B} & \iota_{C \Sigma A} \vee C \iota_{\Sigma B}
\end{array}\right) \circ\left(\begin{array}{cc}
\bar{\rho}_{A, B} \bar{h}_{A, B} & a \\
b & c
\end{array}\right) \\
=\left(\begin{array}{ll}
\nabla \circ(f \vee g) & \nabla \circ\left(f^{\prime} \vee u g\right) \\
\left.\nabla \circ v f \vee g^{\prime \prime}\right) & \nabla \circ\left(v^{\prime} f^{\prime} \vee u^{\prime} g^{\prime \prime}\right)
\end{array}\right) \circ\left(\begin{array}{cc}
\bar{\rho}_{A, B} \bar{h}_{A, B} & a \\
b & c
\end{array}\right) \\
=\left(\partial_{\Phi} \alpha, \partial_{\Phi} T \circ \tau(\beta)\right)_{3}\left(\begin{array}{cc}
\bar{\rho}_{A, B} \bar{h}_{A, B} & a \\
b & c
\end{array}\right) .
\end{aligned}
$$

Thus the desired formula is proved.

The other formulas are proved by using (2.11) and the formulas analogous to (2.11).

Proposition (4.4) [2; Proposition 3.4]. If $A$ and $B$ are suspensions then

$$
\begin{aligned}
& {\left[\alpha, \beta_{1}+\beta_{2}\right]=\left[\alpha, \beta_{1}\right]+\left[\alpha, \beta_{2}\right],} \\
& {\left[\alpha_{1}+\alpha_{2}, \beta\right]=\left[\alpha_{1}, \beta\right]+\left[\alpha_{2}, \beta\right],}
\end{aligned}
$$

where $\alpha, \alpha_{i} \in \pi(\Sigma A, X)$ and $\beta, \beta_{i} \in \pi(\Sigma B, X), i=1,2$.

Proposition (4.5) If $A$ and $B$ are suspensions, then

$$
\begin{aligned}
& {\left[\alpha, \beta_{1}+\beta_{2}\right]_{1}=\left[\alpha, \beta_{1}\right]_{1}+\left[\alpha, \beta_{2}\right]_{1}} \\
& \text { for } \alpha, \alpha_{i} \in \pi_{1}(\Sigma A, k) \text { and } \\
& {\left[\alpha_{1}+\alpha_{2}, \beta\right]_{1}=\left[\alpha_{1}, \beta\right]_{1}+\left[\alpha_{2}, \beta\right]_{1}} \\
& \beta, \beta_{i} \in \pi(\Sigma B, X), i=1,2 \text {, } \\
& {\left[\alpha, \beta_{1}+\beta_{2}\right]_{2}=\left[\alpha, \beta_{1}\right]_{2}+\left[\alpha, \beta_{2}\right]_{2}} \\
& \text { for } \alpha, \alpha_{i} \in \pi_{1}(\Sigma A, k) \text { and } \\
& {\left[\alpha_{1}+\alpha_{2}, \beta\right]_{2}=\left[\alpha_{1}, \beta\right]_{2}+\left[\alpha_{2}, \beta\right]_{2}} \\
& \beta, \beta_{i} \in \pi_{1}(\Sigma B, k), i=1,2 \text {, } \\
& {\left[\alpha, \beta_{1}+\beta_{2}\right]_{3}=\left[\alpha, \beta_{1}\right]_{3}+\left[\alpha, \beta_{2}\right]_{3}} \\
& \text { for } \alpha, \alpha_{i} \in \pi_{1}(\Sigma A, u) \text { and } \\
& {\left[\alpha_{1}+\alpha_{2}, \beta\right]_{3}=\left[\alpha_{1}, \beta\right]_{3}+\left[\alpha_{2}, \beta\right]_{3}} \\
& \beta, \beta_{i} \in \pi_{1}(\Sigma B, v), i=1,2, \\
& {\left[\alpha, \beta_{1}+\beta_{2}\right]_{4}=\left[\alpha, \beta_{1}\right]_{4}+\left[\alpha, \beta_{2}\right]_{4}} \\
& {\left[\alpha_{1}+\alpha_{2}, \beta\right]_{4}=\left[\alpha_{1}, \beta\right]_{4}+\left[\alpha_{2}, \beta\right]_{4}} \\
& \text { for } \alpha, \alpha_{i} \in \pi_{2}(\Sigma A, \Phi) \text { and } \\
& {\left[\alpha, \beta_{1}+\beta_{2}\right]_{5}=\left[\alpha, \beta_{1}\right]_{5}+\left[\alpha, \beta_{2}\right]_{5}} \\
& \beta, \beta_{i} \in \pi(\Sigma B, W), i=1,2 \text {, } \\
& {\left[\alpha_{1}+\alpha_{2}, \beta\right]_{5}=\left[\alpha_{1}, \beta\right]_{5}+\left[\alpha_{2}, \beta\right]_{5}} \\
& \text { for } \alpha, \alpha_{i} \in \pi_{2}(\Sigma A, \Phi) \text { and } \\
& {\left[\alpha, \beta_{1}+\beta_{2}\right]_{6}=\left[\alpha, \beta_{1}\right]_{6}+\left[\alpha, \beta_{2}\right]_{6}} \\
& \beta, \beta_{i} \in \pi_{1}(\Sigma B, u), i=1,2, \\
& {\left[\alpha_{1}+\alpha_{2}, \beta\right]_{6}=\left[\alpha_{1}, \beta\right]_{6}+\left[\alpha_{2}, \beta\right]_{6}} \\
& \text { for } \alpha, \alpha_{i} \in \pi_{2}(\Sigma, \Phi, \Phi) \text { and } \\
& \beta, \beta_{i} \in \pi_{2}(\Sigma B, \Phi), i=1,2 \text {. }
\end{aligned}
$$


PROOF. We shall prove $\left[\alpha, \beta_{1}+\beta_{2}\right]_{1}=\left[\alpha, \beta_{1}\right]_{1}+\left[\alpha, \beta_{2}\right]_{1}$. Let $\alpha=\left\{\left(f_{1}, f_{2}\right)\right\}$, $\beta_{1}=\left\{g_{1}\right\}$ and $\beta_{2}=\left\{g_{2}\right\}$, and let $i_{\Sigma A}: \Sigma A \subset \Sigma A \vee \Sigma B_{1} \vee \Sigma B_{2}, i_{\Sigma B_{1}}: \Sigma B_{1} \subset \Sigma A \vee \Sigma B_{1}$ $\vee \Sigma B_{2}$ and $i_{\Sigma B_{2}}: \Sigma B_{2} \subset \Sigma A \vee \Sigma B_{1} \vee \Sigma B_{2}, B=B_{1}=B_{2}$. Now we consider the commutative diagram

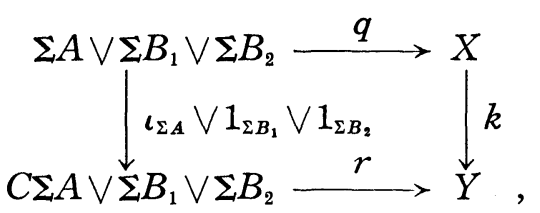

where $q=\nabla \circ(1 \bigvee \nabla) \circ\left(f_{1} \vee g_{1} \vee g_{2}\right)$ and $r=\nabla \circ(1 \vee \nabla) \circ\left(f_{2} \vee k g_{1} \vee k g_{2}\right)$. If $\gamma \in \pi_{1}\left(\Sigma A, \iota_{\Sigma A} \vee 1_{\Sigma B_{1}} \vee 1_{\Sigma B_{z}}\right)$ is an element represented by a map $\left(i_{\Sigma A}, i_{C \Sigma A}\right)$ : $\iota_{\Sigma A} \rightarrow \iota_{\Sigma A} \vee 1_{\Sigma B_{1}} \vee 1_{\Sigma B_{2}}, i_{C \Sigma A}: C \Sigma A \subset C \Sigma A \vee \Sigma B_{1} \vee \Sigma B_{2}$, then we obtain the following formulas :

$$
\left\{\begin{aligned}
(q, r)_{*}\left(\left[\gamma,\left\{i_{\Sigma B_{1}}\right\}+\left\{i_{\Sigma B_{2}}\right\}\right]_{1}\right) & =\left[(q, r)_{*} \gamma, \beta_{1}+\beta_{2}\right]_{1}, \\
(q, r)_{*}\left(\left[\gamma,\left\{i_{\Sigma B_{1}}\right\}\right]_{1}+\left[\gamma,\left\{i_{\Sigma B_{2}}\right\}\right]_{1}\right) & =\left[(q, r)_{*} \gamma, \beta_{1}\right]_{1}+\left[(q, r)_{*} \gamma, \beta_{2}\right]_{1}, \\
{\left[\gamma,\left\{i_{\Sigma B_{1}}\right\}+\left\{i_{\Sigma B_{2}}\right\}\right]_{1} } & =\left[\gamma,\left\{i_{\Sigma B}\right\}\right]_{1}+\left[\gamma,\left\{i_{\Sigma B_{*}}\right\}\right]_{1} .
\end{aligned}\right.
$$

For, the first and second formulas are obtained by (4.1) and the third formula is proved as follows: In the homotopy exact sequence

$$
\begin{aligned}
\longrightarrow & \pi_{1}\left(\Sigma \Lambda, \iota_{\Sigma A} \vee 1_{\Sigma B_{1}} \vee 1_{\Sigma B_{2}}\right) \\
& \stackrel{\partial}{\longrightarrow} \pi\left(\Sigma \Lambda, \Sigma A \vee \Sigma B_{1} \vee \Sigma B_{2}\right) \\
& \stackrel{\left(\iota_{\Sigma A} \vee 1_{\Sigma B_{1}} \vee 1_{\Sigma B_{2}}\right)_{*}}{\longrightarrow} \pi\left(\Sigma \Lambda, C \Sigma A \vee \Sigma B_{1} \vee \Sigma B_{2}\right),
\end{aligned}
$$

$\partial$ is a monomorphism since $\left(\iota_{\Sigma A} \vee 1_{\Sigma B_{1}} \vee 1_{\Sigma B_{2}}\right)$ is onto, and we have

$$
\begin{gathered}
\partial\left[\gamma,\left\{i_{\Sigma B_{1}}\right\}+\left\{i_{\Sigma B_{2}}\right\}\right]_{1}=\left[\partial \gamma,\left\{i_{\Sigma B_{1}}\right\}+\left\{i_{\Sigma B_{2}}\right\}\right] \quad(\text { by }(4.2)), \\
\partial\left(\left[\gamma,\left\{i_{\Sigma B_{1}}\right\}\right]_{1}+\left[\gamma,\left\{i_{\Sigma B_{2}}\right\}\right]_{1}\right)=\left[\partial \gamma,\left\{i_{\Sigma B_{1}}\right\}\right]+\left[\partial \gamma,\left\{i_{\Sigma B_{2}}\right\}\right] \quad(\text { by }(4.2)) .
\end{gathered}
$$

Hence, by using (4.4) and the fact that $\partial$ is a monomorphism, we get desired formula.

Next, by using (4.5.1) and $\alpha=(q, r)_{*} \gamma$, we have

$$
\begin{aligned}
{\left[\alpha, \beta_{1}+\beta_{2}\right]_{1} } & =\left[(q, r)_{*} \gamma, \beta_{1}+\beta_{2}\right]_{1} \\
& =(q, r)_{*}\left(\left[\gamma,\left\{i_{\Sigma B_{1}}\right\}+\left\{i_{\Sigma B_{2}}\right\}\right]_{1}\right) \\
& =(q, r)_{*}\left(\left[\gamma,\left\{i_{\Sigma B_{1}}\right\}\right]_{1}+(q, r)_{*}\left[\gamma,\left\{i_{\Sigma B_{2}}\right\}\right]_{1}\right.
\end{aligned}
$$




$$
\begin{aligned}
& =\left[(q, r)_{*} \gamma, \beta_{1}\right]_{1}+\left[(q, r)_{*} \gamma, \beta_{2}\right]_{1} \\
& =\left[\alpha, \beta_{1}\right]_{1}+\left[\alpha, \beta_{2}\right]_{1} .
\end{aligned}
$$

Similarly we have

$$
\left[\alpha_{1}+\alpha_{2}, \beta\right]_{1}=\left[\alpha_{1}, \beta\right]_{1}+\left[\alpha_{2}, \beta\right]_{1} .
$$

Next we shall prove $\left[\alpha, \beta_{1}+\beta_{2}\right]_{3}=\left[\alpha, \beta_{1}\right]_{3}+\left[\alpha, \beta_{2}\right]_{3}$. Let $\alpha=\left\{\left(f_{1}, f_{2}\right)\right\}$, $\beta_{1}=\left\{\left(g_{1}, g_{1}^{\prime}\right)\right\}$ and $\beta_{2}=\left\{\left(g_{2}, g_{2}^{\prime}\right)\right\}$, and let $i_{C \Sigma B_{1}}: C \Sigma B_{1} \subset \Sigma A \vee C \Sigma B_{1} \vee C \Sigma B_{2}$ and $i_{C \Sigma B_{2}}: C \Sigma B_{2} \subset \Sigma A \vee C \Sigma B_{1} \vee C \Sigma B_{2}$, and let $\Lambda$ be a map such that

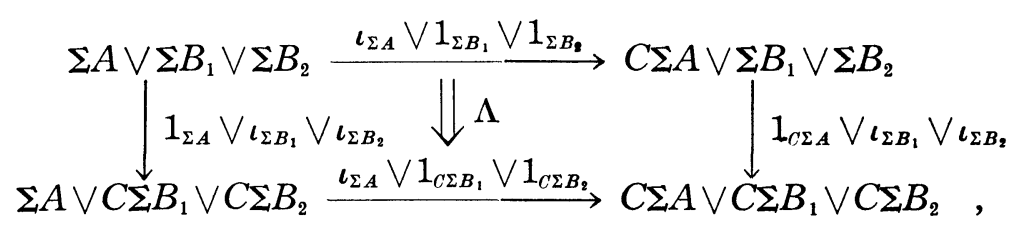

Then we consider the map $\left(\begin{array}{ll}q_{1} & q_{2} \\ r_{1} & r_{2}\end{array}\right): \Lambda \rightarrow \Phi$, where $q_{1}=\nabla \circ(1 \vee \nabla) \circ$ $\left(f_{1} \vee g_{1} \vee g_{2}\right), q_{2}=\nabla \circ(1 \vee \nabla) \circ\left(f_{2} \vee u g_{1} \vee u g_{2}\right), \quad r_{1}=\nabla \circ(1 \vee \nabla) \circ\left(v f_{1} \vee g_{1}^{\prime} \vee g_{2}^{\prime}\right)$ and $r_{2}=\nabla \circ(1 \vee \nabla)\left(v^{\prime} f_{2} \vee u^{\prime} g_{1}^{\prime} \bigvee u^{\prime} g_{2}^{\prime}\right)$. Now if $\gamma_{1}, \gamma_{2} \in \pi_{1}\left(\Sigma B, 1_{\Sigma A} \vee \iota_{\Sigma B_{1}} \vee \iota_{\Sigma B_{2}}\right)$ and $\delta \in \pi_{1}\left(\Sigma A, \iota_{\Sigma A} \vee 1_{\Sigma B_{1}} \vee 1_{\Sigma B_{1}}\right)$ are the elements represented by maps $\left(i_{\Sigma B_{1}}\right.$, $\left.i_{C \Sigma B_{1}}\right): \iota_{\Sigma B_{1}} \rightarrow 1_{\Sigma A} \vee \iota_{\Sigma B_{1}} \vee \iota_{\Sigma B_{2}},\left(i_{\Sigma B_{2}}, i_{C \Sigma B_{2}}\right): \iota_{\Sigma B_{2}} \rightarrow 1_{\Sigma A} \vee \iota_{\Sigma B_{1}} \vee \iota_{\Sigma B_{2}}$ and $\left(i_{\Sigma A}, i_{C \Sigma A}\right):$ $\iota_{\Sigma A} \rightarrow \iota_{\Sigma A} \vee 1_{\Sigma B_{1}} \vee 1_{\Sigma B_{2}}$, respectively then we have the following formulas:

$$
\left\{\begin{aligned}
\left(\begin{array}{ll}
q_{1} & q_{2} \\
r_{1} & r_{2}
\end{array}\right)_{*}\left[\delta, \gamma_{1}+\gamma_{2}\right]_{3} & =\left[\left(q_{1}, q_{2}\right)_{*} \delta, \beta_{1}, \beta_{2}\right]_{3} \\
\left(\begin{array}{ll}
q_{1} & q_{2} \\
r_{1} & r_{2}
\end{array}\right)_{*}\left(\left[\delta, \gamma_{1}\right]_{3}+\left[\delta, \gamma_{2}\right]_{3}\right) & =\left[\left(q_{1}, q_{2}\right)_{*} \delta, \beta_{1}\right]_{3}+\left[\left(q_{1}, q_{2}\right)_{*} \delta, \beta_{2}\right]_{3} \\
{\left[\delta, \gamma_{1}+\gamma_{2}\right]_{3} } & =\left[\delta, \gamma_{1}\right]_{3}+\left[\delta, \gamma_{2}\right]_{3} .
\end{aligned}\right.
$$

We shall prove only the third formula, the other fomulas are obvious.

The boundary homomorphism $\partial_{\Lambda}: \pi_{2}(\Sigma(A \# B), \Lambda) \rightarrow \pi_{1}\left(\Sigma(A \# B), \iota_{\Sigma A}\right.$ $\left.\bigvee 1_{\Sigma B_{1}} \vee 1_{\Sigma B_{2}}\right)$ is a monomorphism, and we have

$$
\begin{aligned}
\partial_{\Lambda}\left[\delta, \gamma_{1}+\gamma_{2}\right]_{3} & =\left[\delta, \partial \gamma_{1}+\partial \gamma_{2}\right]_{1} \\
\partial_{\mathbf{\Lambda}}\left(\left[\delta, \gamma_{1}\right]_{3}+\left[\delta, \gamma_{2}\right]_{3}\right) & =\left[\delta, \partial \gamma_{1}\right]_{1}+\left[\delta, \partial \gamma_{2}\right]_{1}
\end{aligned}
$$

Hence we get $\left[\delta, \gamma_{1}+\gamma_{2}\right]_{3}=\left[\delta, \gamma_{1}\right]_{3}+\left[\delta, \gamma_{2}\right]_{3}$.

By using (4.5.2) we obtain the desired formula $\left[\alpha, \beta_{1}+\beta_{2}\right]_{3}=\left[\alpha, \beta_{1}\right]_{3}+\left[\alpha, \beta_{2}\right]_{3}$. 
If we choose appropriate universal examples, the case $[,]_{4}$ is proved similarly, and the other formulas are proved easily by (4.3).

Next by using Hardie's method [6] we may define the GWP $[\alpha, \beta]_{1}$ as follows: Let $f: \Sigma A \rightarrow X$ and $g: \Sigma B \rightarrow X$ be maps, if we use Hardie's notation $w(f, g)$ is the composition map $\nabla \circ(f \vee g) \circ \bar{\rho}_{A, B} \bar{h}_{A, B}: \Sigma(A \# B) \rightarrow X$.

Consider the commutative diagram

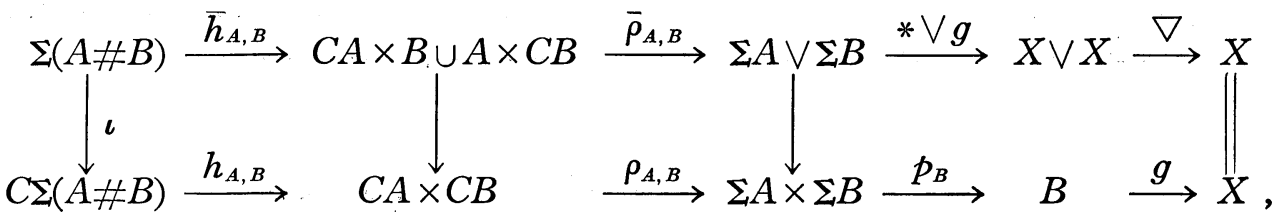

where $p_{B}: \Sigma A \times \Sigma B \rightarrow \Sigma B$ is the projection, and $\rho_{A, B}, h_{A, B}$ are maps defined in [12], and $\lambda_{A}(g)=g \circ p_{B} \circ \rho_{A, B} h_{A, B}$. Let $l: C \Sigma A \rightarrow X$ be a map. Then we define $\bar{w}(l, g): C \Sigma(A \# B) \rightarrow X$ by

$$
\bar{w}(l, g)(y, t)=\left\{\begin{array}{ll}
\lambda_{A}(g)(y, 2 t), & 0 \leqq t \leqq 1 / 2, \\
w\left(l \sigma_{2 t-1}^{\prime}, g\right) y, & 1 / 2 \leqq t \leqq 1,
\end{array} \quad y \in \Sigma(A \# B),\right.
$$

where $\sigma_{2 t-1}^{\prime}: \Sigma A \rightarrow C \Sigma A$ is defined by $\sigma_{2 t-1}^{\prime}(x)=(x, 2 t-1)$ for $x \in \Sigma A$.

Let $k: X \rightarrow Y$ be a map. And if $\left\{\left(f, f^{\prime}\right)\right\}=\alpha \in \pi_{1}(\Sigma A, k)$ and $\{g\}$ $=\beta \in \pi(\Sigma B, X)$, then $\bar{w}\left(f^{\prime}, k g\right): C \Sigma(A \# B) \rightarrow Y$ is given by

$$
\bar{w}\left(f^{\prime}, k g\right)(y, t)=\left\{\begin{array}{ll}
\lambda_{A}(k g)(\mathrm{y}, 2 t), & 0 \leqq t \leqq 1 / 2, \\
w\left(f^{\prime} \sigma_{2 t-1}^{\prime}, k g\right) y, & 1 / 2 \leqq t \leqq 1 .
\end{array} \quad y \in \Sigma(A \# B),\right.
$$

And the diagram

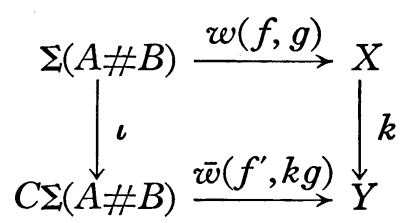

is commutative.

Theorem (4.6) $\left\{w(f, g), \bar{w}\left(f^{\prime}, k g\right)\right\}=[\alpha, \beta]_{1} \in \pi_{1}(\Sigma(A \# B), k)$. 
ProOF. Let $i_{\Sigma A}: \Sigma A \subset \Sigma A \vee \Sigma B, i_{\Sigma B}: \Sigma B \subset \Sigma A \vee \Sigma B$ and $i_{C \Sigma A}: C \Sigma A$ $\subset C \Sigma A \vee \Sigma B$. If we choose $\left\{\left(w\left(i_{\Sigma A}, i_{\Sigma B}\right), \bar{w}\left(i_{C \Sigma A},\left(\iota_{\Sigma A} \vee 1_{\Sigma A}\right) i_{\Sigma B}\right)\right\} \in \pi_{1}\left(\Sigma(A \# B), \iota_{\Sigma A}\right.\right.$ $\left.\vee 1_{\Sigma B}\right)$ we have $\partial_{l_{\Sigma A} \vee 1_{\Sigma B}}\left\{\left(w\left(i_{\Sigma A}, i_{\Sigma B}\right), \bar{w}\left(i_{c \Sigma A},\left(\iota_{\Sigma A} \vee 1_{\Sigma B}\right) i_{\Sigma B}\right)\right\}=\left\{w\left(i_{\Sigma A}, i_{\Sigma B}\right)\right\}=\theta\right.$. Since $\partial_{\iota_{\Sigma A} \vee 1_{\Sigma B}}$ is a monomorphism we obtain $\left\{\left(w\left(i_{\Sigma A}, i_{\Sigma B}\right), \bar{w}\left(i_{C \Sigma A},\left(\iota_{\Sigma A} \vee 1_{\Sigma B}\right) i_{\Sigma B}\right)\right\}\right.$ $=\partial_{\iota_{\Sigma A} \vee 1_{\Sigma B}}^{-1}(\theta)=\theta_{1}=\left\{\left(\bar{\rho}_{A, B} \bar{h}_{A, B}, a\right)\right\}$, thus we deduce $(\alpha, \beta)_{1 *}\left(w\left(i_{\Sigma A} \vee i_{\Sigma B}\right), \bar{w}\left(i_{C \Sigma A}\right.\right.$, $\left.\left.\left(\iota_{\Sigma A} \vee 1_{\Sigma B}\right) i_{\Sigma B}\right)\right\}=(\alpha, \beta)_{1 *}\left(\theta_{1}\right)=[\alpha, \beta]_{1}$.

Hence we now proceed to prove

$$
(\alpha, \beta)_{1 *}\left\{\left(w\left(i_{\Sigma A} \bigvee i_{\Sigma B}\right), \bar{w}\left(i_{C \Sigma A},\left(\iota_{\Sigma A} \bigvee 1_{\Sigma A}\right) i_{\Sigma B}\right)\right\}=\left\{w(f, g), \bar{w}\left(f^{\prime}, k g\right)\right\} .\right.
$$

The left hand side of the above formula is $\left\{\left(\nabla \circ(f \vee g) \circ \bar{\rho}_{A, B} \bar{h}_{A, B}\right.\right.$, $\nabla \circ\left(f^{\prime} \vee k g\right) \circ \bar{w}\left(i_{C \Sigma A},\left(\iota_{\Sigma A} \bigvee 1_{\Sigma B}\right) i_{\Sigma B}\right)$ and

$$
\begin{aligned}
\nabla \circ\left(f^{\prime} \vee k g\right) & \bar{w}\left(i_{C \Sigma A},\left(\iota_{\Sigma A} \vee 1_{\Sigma B}\right) i_{\Sigma B}\right) \\
& = \begin{cases}\left.\nabla\left(f^{\prime} \vee k g\right) \lambda_{A}\left(\iota_{\Sigma A} \vee 1_{\Sigma B}\right) i_{\Sigma B}\right)(y, 2 t), & 0 \leqq t \leqq 1 / 2, \\
\nabla\left(f^{\prime} \vee k g\right) w\left(i_{C \Sigma A} \sigma_{2 t-1}^{\prime},\left(\iota_{\Sigma A} \vee 1_{\Sigma B}\right) i_{\Sigma B}\right) y, & 1 / 2 \leqq t \leqq 1,\end{cases} \\
& = \begin{cases}\lambda_{A}(k g)(y, 2 t), & 0 \leqq t \leqq 1 / 2, \\
w\left(f^{\prime} \sigma_{2 t-1}^{\prime}, k g\right) y, & 1 / 2 \leqq t \leqq 1,\end{cases} \\
& =\bar{w}\left(f^{\prime}, k g\right)(y, t) .
\end{aligned}
$$

Therefore we have the desired result.

Lemma (4. 7). [2; Proposition 3.3].

$$
[\beta, \alpha]=-(\Sigma \sigma)^{*}[\alpha, \beta] \text {, for } \alpha \in \pi(\Sigma A, X) \text { and } \beta \in \pi(\Sigma B, X) \text {, }
$$

where $\sigma: B \# A \rightarrow A \# B$ is induced by a map $B \times A \rightarrow A \times B$ defined by $(y, x) \rightarrow(x, y)$ for $x \in A$ and $y \in B$.

Consider maps $\left(\Sigma \sigma, C \Sigma \sigma^{\prime}\right): \iota_{B, A} \rightarrow \iota_{A, B},\left(\begin{array}{ll}\Sigma \sigma & C \Sigma \sigma \\ C \Sigma \sigma & C^{2} \Sigma \sigma\end{array}\right): \Pi_{B, A}=\left(\iota_{B, A}, \iota_{C_{B, A}}\right)$ $\rightarrow \Pi_{A, B}=\left(\iota_{A, B}, \iota_{C_{A, B}}\right):$

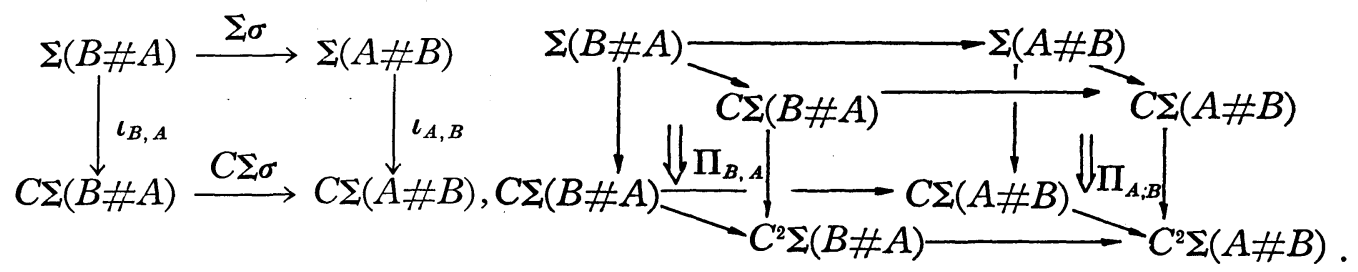


Then we have

THEOREM (4.8).

$[\beta, \alpha]_{1}=-\left(\Sigma o^{\circ}, C \Sigma o^{*}\right)^{*}[\alpha, \beta]_{1} \quad$ for $\quad \alpha \in \pi_{1}(\Sigma A, k)$ and $B \in \pi_{1}(\Sigma B, X)$, $[\beta, \alpha]_{2}=-\left(\Sigma o, C \Sigma \sigma^{*}\right) *[\alpha, \beta]_{2} \quad$ for $\alpha \in \pi_{1}(\Sigma A, k)$ and $B \in \pi_{1}(\Sigma B, k)$, $[\beta, \alpha]_{3}=-\left(\begin{array}{ll}\Sigma \sigma & C \Sigma \sigma \\ C \Sigma \sigma \cdot & C^{2} \Sigma \sigma\end{array}\right)^{*}[\alpha, \beta]_{3} \quad$ for $\quad \alpha \in \pi_{1}(\Sigma A, u)$ and $\beta \in \pi_{1}(\Sigma B, v)$, $[\beta, \alpha]_{4}=-\left(\begin{array}{ll}\Sigma \sigma & C \Sigma \sigma \\ C \Sigma \sigma & C^{2} \Sigma \sigma\end{array}\right)^{*}[\alpha, \beta]_{4} \quad$ for $\quad \alpha \in \pi_{2}(\Sigma A, \Phi)$ and $\beta \in \pi_{2}(\Sigma B, W)$, $[\beta, \alpha]_{5}=-\left(\begin{array}{ll}\Sigma \sigma & C \Sigma \sigma \\ C \Sigma \sigma & C^{2} \Sigma o\end{array}\right)^{*}[\alpha, \beta]_{5} \quad$ for $\quad \alpha \in \pi_{2}(\Sigma A, \Phi)$ and $\quad \beta \in \pi_{1}(\Sigma B, u)$, $[\beta, \alpha]_{b}=-\left(\begin{array}{ll}\Sigma \sigma & C \Sigma \sigma \\ C \Sigma \sigma & C^{2} \Sigma \sigma^{*}\end{array}\right)^{*}[\alpha, \beta]_{B} \quad$ for $\quad \alpha \in \pi_{2}(\Sigma A, \Phi)$ and $B \in \pi_{2}(\Sigma B, \Phi)$.

PROOF. We shall prove the first formula. Let $\alpha=\left\{\left(f, f^{\prime}\right)\right\}$ and $\beta=\{g\}$ and let

$$
\begin{array}{lll}
i_{1, \Sigma A}: \Sigma A \subset \Sigma A \vee \Sigma B, & i_{1, \Sigma B}: \Sigma B \subset \Sigma A \vee \Sigma B, & i_{1, C \Sigma A}: C \Sigma A \subset C \Sigma A \vee \Sigma B, \\
i_{2, \Sigma A}: \Sigma A \subset \Sigma B \vee \Sigma A, & i_{2, \Sigma B}: \Sigma B \subset \Sigma B \vee \Sigma A, & i_{2, C \Sigma A}: C \Sigma A \subset \Sigma B \vee C \Sigma A .
\end{array}
$$

Consider maps

$$
\begin{array}{ll}
p_{1}=\nabla \circ(f \vee g): \Sigma A \vee \Sigma B \longrightarrow X, & q_{1}=\nabla \circ\left(f^{\prime} \vee k g\right): C \Sigma A \vee \Sigma B \longrightarrow Y, \\
p_{2}=\nabla \circ(g \vee f): \Sigma B \vee \Sigma A \longrightarrow X, & q_{2}=\nabla \circ\left(k g \vee f^{\prime}\right): \Sigma B \vee C \Sigma A \longrightarrow Y .
\end{array}
$$

Let $o_{U \times V}: V \times U \rightarrow U \times V$ be the map by $(v, u) \rightarrow(u, v)$ and let $\sigma_{U \vee V}$ $=\sigma_{U \times V} \mid V \vee U: V \vee U \rightarrow U \vee V$, where $U$ and $V$ are any spaces. Then $\left(\Sigma \sigma_{A \bigvee B}, \Sigma \sigma_{C A \bigvee B}\right): 1_{\Sigma B} \vee \iota_{\Sigma A} \rightarrow \iota_{\Sigma A} \vee 1_{\Sigma B}$ is a map of pair as follows :

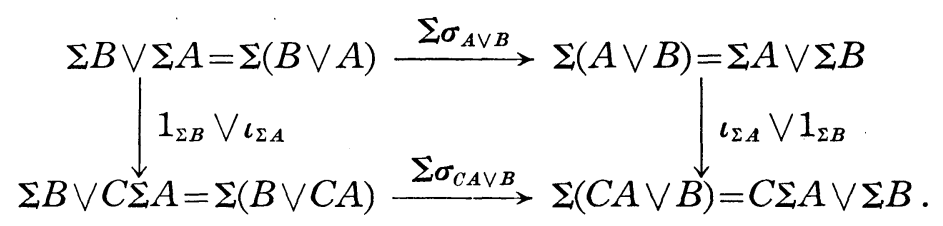

Let $\gamma_{1}=\left\{\left(i_{1, \Sigma A}, i_{1, c \Sigma A}\right)\right\} \in \pi_{1}\left(\Sigma A, \iota_{\Sigma A} \bigvee 1_{\Sigma B}\right)$ and $\gamma_{2}=\left\{\left(i_{2, \Sigma A}, i_{2, c \Sigma A}\right)\right\} \in \pi_{1}\left(\Sigma A, 1_{\Sigma B} \vee \iota_{\Sigma A}\right)$. Then we have 
(4. 8. 1) $\left(\Sigma \sigma_{A \bigvee B}, \Sigma \sigma_{C A \bigvee B}\right)_{*}\left[\left\{i_{2, \Sigma B}\right\}, \gamma_{2}\right]_{1}+(\Sigma \sigma, C \Sigma \sigma)^{*}\left[\left(\Sigma \sigma_{A \bigvee B}, \Sigma \sigma_{C A \vee B}\right)_{*}\left(\gamma_{2}\right),\left\{i_{1, \Sigma B}\right\}\right]_{1}=0$

This is proved as follows :

$$
\begin{aligned}
& \partial_{l_{\Sigma A} \vee 1_{\Sigma B}}\left\{\left(\Sigma \sigma_{A \vee B}, \Sigma \sigma_{C A \vee B}\right)_{*}\left[\left\{i_{2, \Sigma B}\right\}, \gamma_{2}\right]_{1}+(\Sigma \sigma, C \Sigma \sigma)^{*}\left[\Sigma \sigma_{A \vee B}, \Sigma \sigma_{C A \vee B}\right)_{*}\left(\gamma_{2}\right),\left\{i_{1, \Sigma B}\right\}\right]_{1} \\
& =\left[\left(\Sigma \sigma_{A \bigvee B}\right)_{*}\left\{i_{2, \Sigma B}\right\},\left(\Sigma \sigma_{A \vee B}\right)_{*} \circ \partial_{1_{\Sigma B} \vee_{\varepsilon A}}\left(\gamma_{2}\right)\right] \\
& +(\Sigma \sigma)^{*} \circ \partial_{\iota_{\Sigma A} \vee 1_{\Sigma B}}\left[\left(\Sigma \sigma_{A \vee B}, \Sigma \sigma_{C A \vee B}\right)_{*}\left(\gamma_{2}\right),\left\{i_{1, \Sigma B}\right\}\right]_{1} \\
& =\left[\left\{i_{1, \Sigma B}\right\},\left(\Sigma \sigma_{A \vee B}\right)_{*} \circ \partial_{1_{\Sigma B} \vee \iota_{\Sigma A}}\left(\gamma_{2}\right)\right]+(\Sigma \sigma)^{*}\left[\left(\Sigma \sigma_{A \vee B}\right)_{*} \circ \partial_{1_{\Sigma B} \vee t_{\Sigma} A}\left(\gamma_{2}\right),\left\{i_{1, \Sigma B}\right\}\right] \\
& =0 \text { (by (4.7)). }
\end{aligned}
$$

Since $\partial_{t_{\Sigma A} \vee 1_{\Sigma B}}$ is monomorphic we obtain the desired formula.

Then we have

$$
\begin{aligned}
\left(p_{1}, q_{1}\right)_{*}\left(\Sigma \sigma_{A \bigvee B}, \Sigma \sigma_{C A \bigvee B}\right)_{*}\left[\left\{i_{2, \Sigma B}\right\}, \gamma_{2}\right]_{1} & =\left(p_{2}, q_{2}\right)_{*}\left[\left\{i_{2, \Sigma B}\right\}, \gamma_{2}\right]_{1} \\
& =\left[p_{2}\left\{i_{2, \Sigma B}\right\},\left(p_{2}, q_{2}\right)_{*} \gamma_{2}\right]_{1} \quad(\text { by }(4.1)) \\
& =[\beta, \alpha]_{1} .
\end{aligned}
$$

On the other hand

$$
\begin{aligned}
\left.\left(p_{1}, q_{1}\right)_{*}(\Sigma \sigma, C \Sigma \sigma)^{*}\left[\Sigma \sigma_{A \bigvee B}, \Sigma \sigma_{C A \bigvee B}\right)_{*}\left(\gamma_{2}\right),\left\{i_{1, \Sigma B}\right\}\right]_{1} \\
=(\Sigma \sigma, C \Sigma \sigma)^{*}\left(p_{1}, q_{1}\right)_{*}\left[\left(\sum \sigma_{A \bigvee B}, \Sigma \sigma_{C A \bigvee B}\right)_{*}\left(\gamma_{2}\right),\left\{i_{1, \Sigma B}\right\}\right]_{1} \\
=(\Sigma \sigma, C \Sigma \sigma)^{*}\left[\left(p_{1}, q_{1}\right)_{*}\left(\Sigma \sigma_{A \bigvee B}, \Sigma \sigma_{C A \bigvee B}\right)_{*}\left(\gamma_{2}\right), p_{1 *}\left\{i_{1, \Sigma B}\right\}\right]_{1} \\
=(\Sigma \sigma, C \Sigma \sigma)^{*}\left[\left(p_{1}, q_{1}\right)_{*} \gamma_{2}, \beta\right]_{1} \\
=(\Sigma \sigma, C \Sigma o)^{*}[\alpha, \beta]_{1} .
\end{aligned}
$$

Hence, by (4.8.1) we have $[\beta, \alpha]_{1}=-(\Sigma \sigma, C \Sigma \sigma)^{*}[\alpha, \beta]_{1}$.

Next we shall prove the third formula. Let $\alpha=\left\{\left(f, f^{\prime}\right)\right\}$ and $\beta=\left\{\left(g, g^{\prime}\right)\right.$, and let $i_{1, c \Sigma B}: C \Sigma B \subset \Sigma A \vee C \Sigma B$ and $i_{2, c \Sigma B}: C \Sigma B \subset C \Sigma B \vee \Sigma A$. We consider the $\operatorname{map}\left(\begin{array}{cc}p_{1} & q_{1} \\ r_{1} & s_{1}\end{array}\right): \Psi_{A, B} \rightarrow \Phi$, where $p_{1}=\nabla \circ(f \vee g), q_{1}=\nabla \circ\left(f^{\prime} \vee u g\right), \quad r_{1}$ $=\nabla \circ\left(v f \vee g^{\prime}\right)$ and $s_{1}=\nabla \circ\left(v^{\prime} f \vee u^{\prime} g^{\prime}\right), \Psi_{A, B}=\Psi$. Similarly we set $p_{2}=\nabla \circ(g \vee f)$, $q_{2}=\nabla \circ\left(u g \vee f^{\prime}\right), r_{2}=\nabla \circ\left(g^{\prime} \vee v f\right)$ and $s_{2}=\nabla \circ\left(u^{\prime} g^{\prime} \vee v^{\prime} f\right)$.

Let $\Theta_{A, B}, \Theta_{B, A}$ be the maps of pair $\left(\Sigma\left(1_{A} \bigvee \iota_{B}\right), \Sigma\left(\iota_{C A} \bigvee \iota_{B}\right)\right): \Sigma\left(\iota_{A} \bigvee 1_{B}\right) \rightarrow \Sigma\left(\iota_{A}\right.$ $\left.\bigvee 1_{C B}\right),\left(\Sigma\left(\iota_{B} \bigvee 1_{A}\right),\left(\Sigma\left(\iota_{B} \bigvee 1_{C A}\right)\right): \Sigma\left(1_{B} \bigvee \iota_{A}\right) \rightarrow \Sigma\left(1_{C B} \bigvee \iota_{B}\right):\right.$

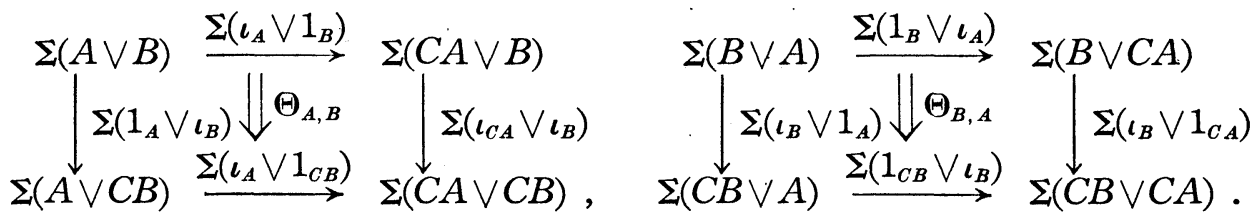


Then $\Theta_{A, B}$ and $\Theta_{B, A}$ are identified with $\Psi_{A, B}$ and $\Psi_{B, A}$ respectively, in the obvious way, where $\Psi_{B, A}=\Psi^{\prime}$. Consider a map of pair of pair $\left(\begin{array}{ll}\Sigma \sigma_{A \vee B} & \Sigma \sigma_{C A \vee B} \\ \Sigma \sigma_{A \vee C B} & \Sigma \sigma_{C A \vee C B}\end{array}\right)$ : $\Psi_{B, A}=\Theta_{B, A} \rightarrow \Theta_{A, B}=\Psi_{A, B}, \quad$ and let $\gamma_{1}=\left\{\left(i_{1, \Sigma A}, i_{1, C \Sigma A}\right)\right\} \in \pi_{1}\left(\Sigma A, \iota_{\Sigma A} \vee 1_{\Sigma B}\right)$, $\gamma_{2}=\left\{\left(i_{1, \Sigma B}, i_{1, C \Sigma B}\right)\right\} \in \pi_{1}\left(\Sigma B, 1_{\Sigma A} \bigvee \iota_{\Sigma B}\right), \gamma_{1}^{\prime}=\left\{\left(i_{2, \Sigma B}, i_{2, C \Sigma B}\right)\right\} \in \pi_{1}\left(\Sigma B, \iota_{\Sigma B} \bigvee 1_{\Sigma A}\right)$ and $\gamma_{2}^{\prime}=\left\{\left(i_{2, \Sigma A}, i_{2, C \Sigma A}\right)\right\} \in \pi_{1}\left(\Sigma A, 1_{\Sigma B} \bigvee \iota_{\Sigma A}\right)$. Then we have

$$
\left(\begin{array}{ll}
\Sigma \sigma_{A \bigvee B} & \Sigma \sigma_{C A \vee B} \\
\Sigma \sigma_{A \vee C B} & \Sigma \sigma_{C A \vee C B}
\end{array}\right)_{*}\left[\gamma_{1}^{\prime}, \gamma_{2}^{\prime}\right]_{3}+\left(\begin{array}{cc}
\Sigma \sigma & C \Sigma \sigma \\
C \Sigma \sigma & C{ }^{2} \Sigma \sigma
\end{array}\right)^{*}\left[\left(\Sigma \sigma_{A \vee B}, \Sigma \sigma_{C A \vee B}\right)_{*}\left(\gamma_{2}^{\prime}\right), \gamma_{2}\right]_{3}=0 .
$$

This is proved as follows :

$$
\begin{aligned}
& \partial_{\Psi_{A, B}} \text { (the left hand side of (4.8.2)) } \\
& =\partial_{\Psi_{A, B}}\left[\left(\Sigma \sigma_{A \vee B}, \Sigma \sigma_{A \vee C B}\right)_{*}\left(\gamma_{1}^{\prime}\right),\left(\Sigma \sigma_{A \bigvee B}, \Sigma \sigma_{C A \vee B}\right)_{*}\left(\gamma_{2}^{\prime}\right)\right]_{3} \\
& +(\Sigma \sigma, C \Sigma \sigma)^{*} \partial_{\Psi_{A, B}}\left[\left(\Sigma \sigma_{A \vee B}, \Sigma \sigma_{C A \vee B}\right)_{*}\left(\gamma_{2}^{\prime}\right), \gamma_{2}\right]_{3} \\
& =\left[\partial_{1_{\Sigma A} \vee \iota_{\Sigma B}}\left(\Sigma \sigma_{A \vee B}, \Sigma \sigma_{A \vee C B}\right)_{*}\left(\gamma_{1}^{\prime}\right),\left(\Sigma \sigma_{A \vee B}, \Sigma \sigma_{C A \vee B}\right)_{*}\left(\gamma_{2}^{\prime}\right)\right]_{1} \\
& +(\Sigma \sigma, C \Sigma \sigma)^{*}\left[\left(\Sigma \sigma_{A \vee B}, \Sigma \sigma_{C A \vee B}\right)_{*}\left(\gamma_{2}^{\prime}\right), \partial_{1_{\Sigma A} \vee \iota_{\Sigma B}}\left(\gamma_{2}\right)\right]_{1} \\
& =\left[\left(\Sigma \sigma_{A \bigvee B}\right)_{*}\left\{i_{2, \Sigma B}\right\},\left(\Sigma \sigma_{A \vee B}, \Sigma \sigma_{C A \vee B}\right)_{*}\left(\gamma_{2}^{\prime}\right)\right]_{1} \\
& +(\Sigma \sigma, C \Sigma \sigma)^{*}\left[\left(\Sigma \sigma_{A \bigvee B}, \Sigma \sigma_{C A \backslash B}\right)^{*}\left(\gamma_{2}^{\prime}\right),\left\{i_{1, \Sigma B}\right\}\right]_{1} \\
& =\left[\left\{i_{1, \Sigma B}\right\},\left(\Sigma \sigma_{A \bigvee B}, \Sigma \sigma_{C A \bigvee B}\right)_{*}\left(\gamma_{2}^{\prime}\right)\right]_{1}+(\Sigma \sigma, C \Sigma \sigma)^{*}\left[\left(\Sigma \sigma_{A \bigvee B}, \Sigma \sigma_{C A \bigvee B}\right)_{*}\left(\gamma_{2}^{\prime}\right),\left\{i_{1, \Sigma B}\right]_{1}\right. \\
& =0 \text {. }
\end{aligned}
$$

Since $\partial_{\Psi_{A, B}}: \pi_{2}\left(\Sigma(B \# A), \Psi_{A, B}\right) \rightarrow \pi_{1}\left(\Sigma(B \# A), \iota_{\Sigma A} \vee 1_{\Sigma B}\right)$ is monomorphic we obtain the desired formula.

Next we have

$$
\begin{aligned}
\left(\begin{array}{ll}
p_{1} & q_{1} \\
r_{1} & s_{1}
\end{array}\right)_{*}\left(\begin{array}{ll}
\Sigma \sigma & C \Sigma \sigma \\
C \Sigma \sigma & C^{2} \Sigma \sigma
\end{array}\right)^{*}\left[\left(\Sigma \sigma_{A, B}, \Sigma \sigma_{C A \vee B}\right)_{*}\left(\gamma_{2}^{\prime}\right), \gamma_{2}\right]_{3} \\
=\left(\begin{array}{ll}
\Sigma \sigma & C \Sigma \sigma \\
C \Sigma \sigma & C^{2} \Sigma \sigma
\end{array}\right)^{*} \circ\left(\begin{array}{ll}
p_{1} & q_{1} \\
r_{1} & s_{1}
\end{array}\right)_{*}\left[\left(\Sigma \sigma_{A, B}, \Sigma \sigma_{C A \vee B}\right)_{*}\left(\gamma_{2}^{\prime}\right), \gamma_{2}\right]_{3} \\
=\left(\begin{array}{ll}
\Sigma \sigma & C \Sigma \sigma \\
C \Sigma \sigma & C^{2} \Sigma \sigma
\end{array}\right)^{*}\left[\left(p_{1}, q_{1}\right)_{*}\left(\Sigma \sigma_{A \vee B}, \Sigma \sigma_{C A \vee B}\right)_{*}\left(\gamma_{2}^{\prime}\right), \beta\right]_{3} \\
=\left(\begin{array}{ll}
\Sigma \sigma & C \Sigma \sigma \\
C \Sigma \sigma & C^{2} \Sigma \sigma
\end{array}\right)^{*}\left[\left(p_{2}, q_{2}\right)_{*}\left(\gamma_{2}^{\prime}\right), \beta\right]_{3}
\end{aligned}
$$




$$
=\left(\begin{array}{ll}
\Sigma \sigma & C \Sigma \sigma \\
C \Sigma \sigma & C^{2} \Sigma \sigma
\end{array}\right)^{*}[\alpha, \beta]_{3},
$$

while we deduce

$$
\begin{aligned}
\left(\begin{array}{ll}
p_{1} & q_{1} \\
r_{1} & s_{2}
\end{array}\right)_{*}\left(\begin{array}{ll}
\Sigma \sigma_{A \vee B} & \Sigma \sigma_{C A \vee B} \\
\Sigma \sigma_{C A \bigvee B} & \Sigma \sigma_{C A \bigvee C B}
\end{array}\right)_{*}\left[\gamma_{1}^{\prime} \cdot \gamma_{2}^{\prime}\right]_{3} & =\left(\begin{array}{ll}
p_{2} & q_{2} \\
r_{2} & s_{2}
\end{array}\right)_{*}\left[\gamma_{1}^{\prime}, \gamma_{2}^{\prime}\right]_{3} \\
& =\left[\left(p_{2}, r_{2}\right)_{*}\left(\gamma_{1}^{\prime}\right),\left(p_{2}, q_{2}\right)_{*}\left(\gamma_{2}^{\prime}\right)\right]_{3} \\
& =[\beta, \alpha]_{3} .
\end{aligned}
$$

Hence we have $[\beta, \alpha]_{3}=-\left(\begin{array}{cc}\Sigma \sigma & C \Sigma \sigma \\ C \Sigma \sigma & C^{2} \Sigma \sigma\end{array}\right)^{*}[\alpha, \beta]_{3}$ by (4. 8.2).

If we choose appropriate universal examples, then other formulas are proved in the same way.

LEMMA (4.9) [10; Lemma 16.5']. Let $f: X \rightarrow A \vee B$ be a map, and let $p_{A}\left(p_{B}\right): A \vee B \rightarrow A(B)$ be projection. If $p_{A} \circ f \simeq *$ and $p_{B} f \simeq *$, then $\Sigma f \simeq *$.

Let $\{f\} \in \pi(\Sigma(A \# B), X), \quad\left\{\left(g, g^{\prime}\right)\right\} \in \pi_{1}(\Sigma(A \# B), k)$ and $\left\{\left(\begin{array}{ll}a_{1} & a_{2} \\ b_{1} & b_{2}\end{array}\right)\right\}$ $\epsilon \pi_{2}(\Sigma(A \# B), \Phi)$. Then suspension homomorphisms

$$
\begin{aligned}
& \Sigma_{X}: \pi(\Sigma(A \# B), X) \longrightarrow \pi\left(\Sigma^{2}(A \# B), \Sigma X\right), \\
& \Sigma_{k}: \pi_{1}(\Sigma(A \# B), k) \longrightarrow \pi_{1}\left(\Sigma^{2}(A \# B), \Sigma k\right), \\
& \Sigma_{\Phi}: \pi_{2}(\Sigma(A \# B), \Phi) \longrightarrow \pi_{2}\left(\Sigma^{2}(A \# B), \Sigma \Phi\right)
\end{aligned}
$$

are defined by $\Sigma_{X}\{f\}=\{\Sigma f\}, \Sigma_{k}\left\{\left(g, g^{\prime}\right)\right\}=\left\{\left(\Sigma g, \Sigma g^{\prime}\right)\right\} \quad$ and $\Sigma_{\Phi}\left\{\left(\begin{array}{ll}a_{1} & a_{2} \\ b_{1} & b_{2}\end{array}\right)\right\}$ $=\left\{\left(\begin{array}{ll}\Sigma a_{1} & \Sigma a_{2} \\ \Sigma b_{1} & \Sigma b_{2}\end{array}\right)\right\}$, respectively.

TheOREM (4.10). All GWP's $[\alpha, \beta]$ and $[\alpha, \beta]_{i}, i=1,2,3,4,5,6$ are annihilated by suspension homomorphisms.

PROOF. We now shall prove $\Sigma_{X}[\alpha, \beta]=0$ for $\alpha \in \pi(\Sigma A, X)$ and $\beta \in \pi(\Sigma B, X)$.

We consider the commutative diagram

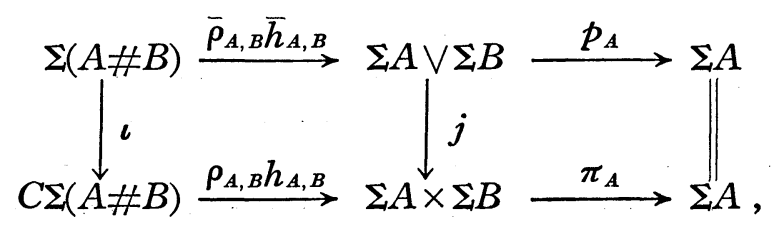


where $j$ is the inclusion map, and $p_{A}$ and $\pi_{A}$ are projections. Since $C \Sigma(A \# B)$ is contractible we have $\pi_{A} \circ\left(\rho_{A, B} h_{A, B}\right)^{\circ} \iota=p_{A} \circ\left(\bar{\rho}_{A, B} \bar{h}_{A, B}\right) \simeq *$. Similarly we have $p_{B} \circ\left(\bar{\rho}_{A, B} \bar{h}_{A, B}\right) \simeq *$. Thus we obtain $\Sigma\left(\bar{\rho}_{A, B} \bar{h}_{A, B}\right) \simeq *$ by Lemma (4.9).

Let $\alpha=\{f\}$ and $\beta=\{g\}$. We consider the commutative diagram

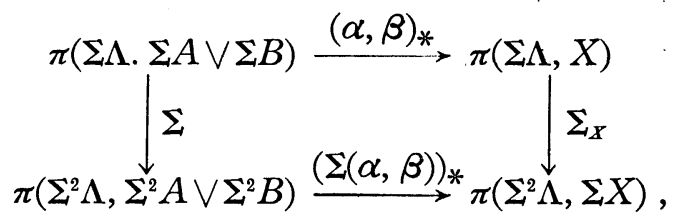

where $(\alpha, \beta)=\nabla \circ(f \vee g)$ and $\Sigma(\alpha, \beta)=\nabla \circ(\Sigma f \vee \Sigma g)$, and $\Sigma \Lambda=\Sigma(A \# B)$. Then $\Sigma_{X}[\alpha, \beta]=\Sigma_{X}(\alpha, \beta)_{*}(\theta)=(\Sigma(\alpha, \beta))_{*} \Sigma(\theta)=0$.

Next we shall prove $\Sigma_{k}[\alpha, \beta]_{1}=0$ for $\alpha=\left\{\left(f, f^{\prime}\right)\right\} \in \pi_{1}(\Sigma A, k)$ and $\beta=\{g\}$ $\epsilon \pi(\Sigma B, X)$ and $\Sigma_{\Phi}[\alpha, \beta]_{3}=0$ for $\alpha=\left\{\left(f, f^{\prime}\right)\right\} \in \pi_{1}(\Sigma A, u)$ and $\beta=\left\{\left(g, g^{\prime}\right)\right\}$ $\epsilon \pi_{1}(\Sigma B, v)$. We consider the commutative diagram

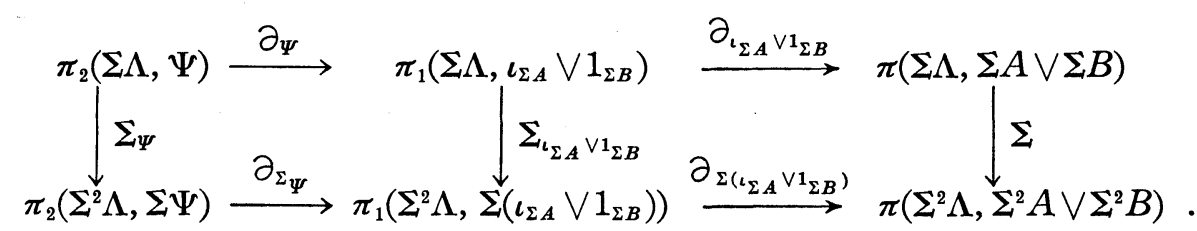

Then $\partial_{\iota_{\Sigma A} \vee 1_{\Sigma B}}, \partial_{\Sigma\left(\iota_{\Sigma A} \vee 1_{\Sigma B}\right)}, \partial_{\Psi}$ and $\partial_{\Sigma_{\Psi}}$ are monomorphisms by ((2.2) (iii)). From the commutativity of the above diagram and $\Sigma(\theta)=0$ we deduce $\Sigma_{c_{\Sigma A} \vee 1_{\Sigma B}}(\theta)_{1}=0$ and $\Sigma_{\Psi}\left(\theta_{3}\right)=0$, and we have $\Sigma_{k}[\alpha, \beta]_{1}=\Sigma_{k} \circ(\alpha, \beta)_{1 *}\left(\theta_{1}\right)$ $=\left(\Sigma(\alpha, \beta)_{1}\right)_{*}\left(\Sigma_{\iota_{\Sigma} \boldsymbol{V} \mathrm{v}_{\Sigma B}}\left(\theta_{1}\right)\right)=0, \Sigma_{\Phi}[\alpha, \beta]_{3}=\Sigma_{\Phi} \circ(\alpha, \beta)_{3 *}\left(\theta_{3}\right)=\left(\left(\Sigma(\alpha, \beta)_{3}\right)_{*}\left(\Sigma_{\Psi} \theta_{3}\right)=0\right.$, where $\Sigma(\alpha, \beta)_{1}=\left(\nabla \circ(\Sigma f \vee \Sigma g), \nabla \circ\left(\Sigma f^{\prime} \vee \Sigma k \circ \Sigma g\right)\right)$ and $\Sigma(\alpha, \beta)_{3}=\left(\begin{array}{l}\nabla(\Sigma f \vee g) \\ \nabla\left(\Sigma v \circ \Sigma f \vee \Sigma g^{\prime}\right)\end{array}\right.$ $\left.\begin{array}{c}\nabla\left(\Sigma f^{\prime} \vee \Sigma u \circ \Sigma g\right) \\ \nabla\left(\Sigma v^{\prime} \circ \Sigma f \vee \Sigma u^{\prime} \circ \Sigma g^{\prime}\right)\end{array}\right)$

The other cases are proved similarly.

5. The Jacobi identity. In [2], Arkowitz described that it is possible to prove the appropriate Jacobi identity for GWP of elements $\alpha \in \pi(\Sigma A, X)$, $\beta \in \pi(\Sigma B, X)$ and $\gamma \in \pi(\Sigma C, X)$, when $A, B$ and $C$ are suspensions.

In this section we represent the Jacobi identity in the explicit form and we consider the appropriate Jacobi identities for other GWP's.

First, we recall the concept mentioned in [3]. Let $p_{A}: \Sigma A \times \Sigma B \rightarrow \Sigma A$ and $p_{B}: \Sigma A \times \Sigma B \rightarrow \Sigma B$ be projections, and let $p_{A}^{*}\left(p_{B}^{*}\right): \pi(\Sigma A, \Omega X)(\pi(\Sigma B, \Omega X))$ $\rightarrow \pi(\Sigma A \times \Sigma B, \Omega X)$ be the homomorphism induced by $p_{A}\left(p_{B}\right)$. And if we choose 
an $\alpha \in \pi(\Sigma A, \Omega X)$ and a $\beta \in \pi(\Sigma B, \Omega X)$ then these determine $p_{A}^{*}(\alpha), p_{B}^{*}(\beta)$ $\epsilon \pi(\Sigma A \times \Sigma B, \Omega X)$. We consider the commutator $\left(p_{A}^{*}(\alpha), p_{B}^{*}(\beta)\right)=\left(p_{A}^{*}(\alpha)^{-1}\right.$ - $\left.p_{B}^{*}(\beta)^{-1}\right) \cdot\left(p_{A}^{*}(\alpha) \cdot p_{B}^{*}(\beta)\right)$ in $\pi(\Sigma A \times \Sigma B, \Omega X)$, and for the cofibration $\Sigma \mathrm{A} \vee \Sigma B$ $\rightarrow \Sigma A \times \Sigma B \rightarrow \Sigma A \# \Sigma B$ there exists an exact sequence

$$
\pi(\Sigma A \# \Sigma B, \Omega X) \stackrel{q^{*}}{\longrightarrow} \pi(\Sigma A \times \Sigma B, \Omega X) \stackrel{j^{*}}{\longrightarrow} \pi(\Sigma A \vee \Sigma B, \Omega X),
$$

where $q$ is the projection. Then $j^{*}\left(p_{A}^{*}(\alpha), p_{B}^{*}(\beta)\right)=0$ and $q^{*}$ is a monomorphism [3], and the commutator product of $\alpha \in \pi(\Sigma A, \Omega X)$ and $\beta \in \pi(\Sigma B, \Omega X)$ is the element $\langle\alpha, \beta\rangle \in \pi(\Sigma A \# \Sigma B, \Omega X)$ uniquely defined by

$$
<\alpha, \beta>=q^{*-1}\left(p_{A}^{*}(\alpha), p_{B}^{*}(\beta)\right) \text {. }
$$

Let $p_{1}: \Sigma A \times \Sigma B \times \Sigma C \rightarrow \Sigma A, \quad p_{2}: \Sigma A \times \Sigma B \times \Sigma C \rightarrow \Sigma B$ and $p_{3}: \Sigma A \times \Sigma B$ $\times \Sigma C \rightarrow \Sigma C$ be projections. Now let $\{f\}=\alpha \in \pi(\Sigma A, \Omega X),\{g\}=\beta \in \pi(\Sigma B, \Omega X)$ and $\{h\}=\gamma \in \pi(\Sigma C, \Omega X)$. Then we obtain maps $f \circ p_{1}=f^{\prime}, g \circ p_{2}=g^{\prime}, h \circ p_{3}=h^{\prime}$ : $\Sigma A \times \Sigma B \times \Sigma C \rightarrow \Omega X$. We consider the map $a=\left(f^{\prime},\left(g^{\prime}, h^{\prime}\right)\right): \Sigma A \times \Sigma B \times \Sigma C$ $\rightarrow \Omega X$, where $\left(g^{\prime}, h^{\prime}\right)=g^{\prime-1} \cdot h^{\prime-1} \cdot g^{\prime} \cdot h^{\prime}$ and the products and inverse in the commutator come from the loop space structure of $\Omega X$. Then we see $a \mid T \simeq *$ : $T \rightarrow \Omega X$ since $a|\Sigma A \times \Sigma B \times * \simeq *, a| \Sigma A \times * \times \Sigma C \simeq *$ and $a \mid * \times \Sigma B \times \Sigma C \simeq *$, where $T=\Sigma A \times \Sigma B \times * \cup \Sigma A \times * \times \Sigma C \cup * \times \Sigma B \times \Sigma C$. For the cofibration $T \stackrel{j_{T}}{\longrightarrow} \Sigma A \times \Sigma B \times \Sigma C \stackrel{p_{1,2,3}}{\longrightarrow} \Sigma A \# \Sigma B \# \Sigma C$ there exists an exact sequence

$$
\pi(\Sigma A \# \Sigma B \# \Sigma C, \Omega X) \stackrel{p_{1,2,3}^{*}}{\longrightarrow} \pi(\Sigma A \times \Sigma B \times \Sigma C, \Omega X) \stackrel{j_{T}^{*}}{\longrightarrow} \pi(T, \Omega X),
$$

where $j_{T}$ is the inclusion map and $p_{1,2,3}$ is the projection. Then $p_{1,2,3}^{*}$ is a monomorphism [3: Proposition 8] and $j_{T}^{*}\{a\}=0$. By using the method analogous to that in [14], we have

PROPOSITION (5.1). $\quad\left\langle\alpha,<\beta, \gamma>>=p_{1,2,3}^{*-1}\left(p_{1}^{*} \alpha,\left(p_{2}^{*} \beta, p_{3}^{*} \gamma\right)\right)\right.$

where $\alpha \in \pi(\Sigma A, \Omega X), \beta \in \pi(\Sigma B, \Omega X)$ and $\gamma \in \pi(\Sigma C, \Omega X)$.

Let $\rho_{B, C, A}: \Sigma A \times \Sigma B \times \Sigma C \rightarrow \Sigma B \times \Sigma C \times \Sigma A$ and $\rho_{C, A, B}: \Sigma A \times \Sigma B \times \Sigma C$ $\rightarrow \Sigma C \times \Sigma A \times \Sigma B$ be the maps given by $\rho_{B, C, A}(x, y, z)=(y, z, x)$ and $\rho_{C, A, B}(x, y, z)$ $=(z, x, y)$ for $x \in \Sigma A, y \in \Sigma B$ and $z \in \Sigma C$, respectively. Then $\rho_{B, C, A}$ and $\rho_{C, A . B}$ induce

$\rho_{B, C, A}^{\prime}: \Sigma A \# \Sigma B \# \Sigma C \rightarrow \Sigma B \# \Sigma C \# \Sigma A, \quad \rho_{C, A, B}^{\prime}: \Sigma A \# \Sigma B \# \Sigma C \rightarrow \Sigma C \# \Sigma A \# \Sigma B$

And we may prove the following propositions [14]: 
PROPOSITION (5.2) $\quad \rho_{B, C, A}^{\prime *}<\beta,<\gamma, \alpha>>=p_{1,2,3}^{*-1}\left(p_{2}^{*}(\beta),\left(p_{3}^{*}(\gamma), p_{1}^{*}(\alpha)\right)\right.$,

$$
\rho_{C, A, B}^{*}<\gamma,\left\langle\alpha, \beta>>=p_{1,2,3}^{*-1}\left(p_{3}^{*}(\gamma),\left(p_{1}^{*}(\alpha), p_{2}^{*}(\beta)\right) .\right.\right.
$$

Proposition (5.3) If $\alpha \in \pi(\Sigma A, \Omega X), \beta \in \pi(\Sigma B, \Omega X)$ and $\gamma \in \pi(\Sigma C, \Omega X)$, then

$$
\left\langle\alpha,\left\langle\beta, \gamma>>+\rho_{B, C, A}^{\prime *}<\beta,\left\langle\gamma, \alpha>>+\rho_{C, A, B}^{*}<\gamma,\langle\alpha, \beta>>=0 .\right.\right.\right.
$$

REMARK. Note that $(X \# Y) \# Z$ and $X \#(Y \# Z)$ are identified and denoted simply $X \# Y \# Z$.

We consider the well-known natural isomorphism $k_{*}: \pi(P, \Omega Q) \rightarrow \pi(\Sigma P, Q)$, defined by $K_{*}\{f\}=\{K(f)\}, K(f)(x, t)=f(x)(t)$ for $x \in P$ and $f: P \rightarrow \Omega Q$. Then it easily follows that $K_{*}<\alpha, \beta>=\left[K_{*}(\alpha), K_{*}(\beta)\right] \in \pi(\Sigma(\Sigma A \# \Sigma B), X)$, and hence from this fact and (5.3) we obtain the Jacobi identity for the absolute GWP :

Theorem (5.4). If $\alpha \in \pi(\Sigma A, X), \beta \in \pi(\Sigma B, X)$ and $\gamma \in \pi(\Sigma C, X)$ and $A$, $B$ and $C$ are suspensions then

$$
[\alpha,[\beta, \gamma]]+\left(\sum \rho_{B, C, A}^{\prime}\right)^{*}[\beta,[\gamma, \alpha]]+\left(\sum \rho_{C, A, B}^{\prime}\right)^{*}[\gamma,[\alpha, \beta]]=0 .
$$

Now we shall consider the Jacobi identies for the various GWP's defined in section 3. We put $\rho=\rho_{B, C, A}^{\prime}$ and $\tau=\rho_{C, A, B}^{\prime}$, and we consider the commutative diagrams
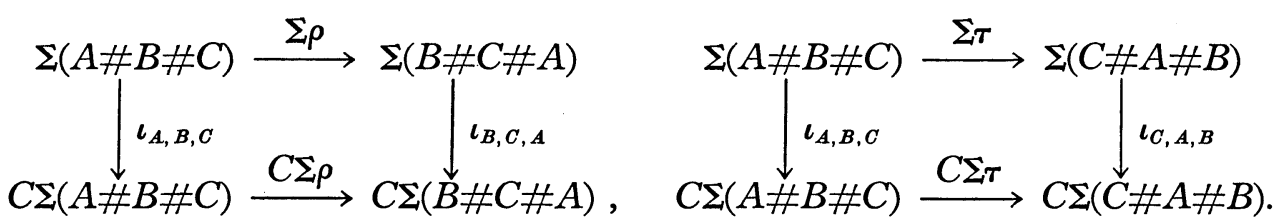

THEOREM (5.5). If $\alpha \in \pi_{1}(\Sigma A, k), \beta \in \pi(\Sigma B, X)$ and $\gamma \in \pi(\Sigma C, X)$, and if $A, B$ and $C$ are suspensions, then

$$
[\alpha,[\beta, \gamma]]_{1}+(\Sigma \rho, C \Sigma \rho)^{*}\left[\beta,[\gamma, \alpha]_{1}\right]_{1}+(\Sigma \tau, C \Sigma \tau)^{*}\left[\gamma,[\alpha, \beta]_{1}\right]_{1}=0 .
$$

PROOF. Let

$$
\begin{aligned}
& i_{1}: \quad \Sigma A \subset \Sigma A \vee \Sigma B \vee \Sigma C, \quad i_{2}: \quad \Sigma B \subset \Sigma A \vee \Sigma B \vee \Sigma C, \\
& i_{3}: \quad \Sigma C \subset \Sigma A \vee \Sigma B \vee \Sigma C, \quad i_{1, C}: C \Sigma A \subset C \Sigma A \vee \Sigma B \vee \Sigma C, \\
& i: \quad \Sigma A \vee \Sigma B \vee \Sigma C \subset C \Sigma A \vee \Sigma B \vee \Sigma C,
\end{aligned}
$$


and let $\iota_{1}=\left\{i_{1}\right\}, \iota_{2}=\left\{i_{2}\right\}, \iota_{3}=\left\{i_{3}\right\}, \alpha=\left\{\left(f, f^{\prime}\right)\right\}, \beta=\{g\}$ and $\gamma=\{h\}$.

We now consider the commutative diagram

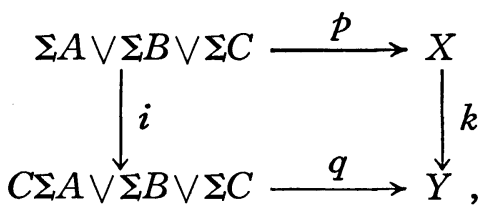

where $p=\nabla \circ(1 \bigvee \nabla) \circ(f \vee g \vee h)$ and $q=\nabla \circ(1 \vee \nabla) \circ\left(f^{\prime} \vee k g \bigvee k h\right)$. If we choose $\iota=\left\{\left(i_{1}, i_{1}, c\right)\right\} \in \pi_{1}(\Sigma A, i)$ then $\left[\iota,\left[\iota_{2}, \iota_{3}\right]\right]_{1} \in \pi_{1}(\Sigma(A \# B \# C), i),\left[\iota_{2},\left[\iota_{3}, \iota\right]_{1}\right]_{1}$ $\in \pi_{1}(\Sigma(B \# C \# A), i)$ and $\left[\iota_{3},\left[\iota, \iota_{2}\right]_{1}\right]_{1} \in \pi_{1}(\Sigma(C \# A \# B), i)$.

Now we shall prove the following formula

$$
\left[\iota,\left[\iota_{2}, \iota_{3}\right]\right]_{1}+(\Sigma \rho, C \Sigma \rho)^{*}\left[\iota_{2},\left[\iota_{3}, \iota\right]_{1}\right]_{1}+(\Sigma \tau, C \Sigma \tau)^{*}\left[\iota_{3},\left[\iota, \iota_{2}\right]_{1}\right]_{1}=0 .
$$

In the homotopy exact sequence

$$
\longrightarrow \pi_{1}(\Sigma \Lambda, i) \stackrel{\partial_{i}}{\longrightarrow} \pi(\Sigma \Lambda, \Sigma A \vee \Sigma B \vee \Sigma C) \stackrel{i_{*}}{\longrightarrow} \pi(\Sigma \Lambda, C \Sigma A \vee \Sigma B \vee \Sigma C)
$$

where $\Sigma \Lambda=\Sigma(A \# B \# C)$, since $i_{*}$ is onto, $\partial_{i}$ is a monomorphism. Hence we obtain

$$
\begin{aligned}
\partial_{i}\left(\left[\iota,\left[\iota_{2}, \iota_{3}\right]\right]_{1}+(\Sigma \rho, C \Sigma \rho)^{*}\left[\iota_{2},\left[\iota_{3}, \iota\right]_{1}\right]_{1}+(\Sigma \tau, C \Sigma \tau)^{*}\left[\iota_{3},\left[\iota, \iota_{2}\right]_{1}\right]_{1}\right) \\
=\left[\partial_{i} \iota,\left[\iota_{2}, \iota_{3}\right]\right]+(\Sigma \rho)^{*} \partial_{i}\left[\iota_{2},\left[\iota_{3}, \iota\right]_{1}\right]_{1}+(\Sigma \tau)^{*} \partial_{i}\left[\iota_{3},\left[\iota, \iota_{2}\right]_{1}\right]_{1} \\
\quad=\left[\iota_{1},\left[\iota_{2}, \iota_{3}\right]\right]+(\Sigma \rho)^{*}\left[\iota_{2}, \partial_{i}\left[\iota_{3}, \iota\right]_{1}\right]+(\Sigma \tau)^{*}\left[\iota_{3}, \partial_{i}\left[\iota, \iota_{2}\right]_{1}\right] \\
=\left[\iota_{1},\left[\iota_{2}, \iota_{3}\right]\right]+(\Sigma \rho)^{*}\left[\iota_{2},\left[\iota_{3}, \iota_{1}\right]\right]+(\Sigma \tau)^{*}\left[\iota_{3},\left[\iota_{1}, \iota_{2}\right]\right] \\
=0 \quad(\text { by }(5.4)) .
\end{aligned}
$$

Since $\partial_{i}$ is a monomorphism we obtain (5.5.1).

Next we have

$$
\begin{array}{r}
(p, q)_{*}\left(\left[\iota,\left[\iota_{2}, \iota_{3}\right]\right]_{1}+(\Sigma \rho, C \Sigma \rho)^{*}\left[\iota_{2},\left[\iota_{3}, \iota\right]_{1}\right]_{1}+(\Sigma \tau, C \Sigma \tau)^{*}\left[\iota_{3},\left[\iota, \iota_{2}\right]_{1}\right]_{1}\right) \\
=\left[(p, q)_{*}, p_{*}\left[\iota_{2}, \iota_{3}\right]\right]_{1}+(\Sigma \rho, C \Sigma \rho)^{*}(p, q)^{*}\left[\iota_{2},\left[\iota_{3}, \iota\right]_{1}\right]_{1} \\
+(\Sigma \tau, C \Sigma \tau)^{*}(p, q)_{*}\left[\iota_{3},\left[\iota, \iota_{2}\right]_{1}\right]_{1} \\
=\left[(p, q)_{*},\left[p_{*} \iota_{2}, p_{*} \iota_{3}\right]\right]_{1}+(\Sigma \rho, C \Sigma \rho)^{*}\left[p_{*},\left[p_{*} \iota_{3},(p, q)_{* \iota}\right]_{1}\right]_{1} \\
+(\Sigma \tau, C \Sigma \tau)^{*}\left[p_{*} \iota_{3},\left[(p, q)_{* \iota}, p^{*} \iota_{2}\right]_{1}\right]_{1}
\end{array}
$$


544

H. ANDO

$$
\begin{aligned}
& =[\alpha,[\beta, \gamma]]_{1}+(\Sigma \rho, C \Sigma \rho)^{*}\left[\beta,[\gamma, \alpha]_{1}\right]_{1}+(\Sigma \tau, C \Sigma \tau)^{*}\left[\gamma,[\alpha, \beta]_{1}\right]_{1} \\
& =0 \quad(\text { by }(5.5 .1)) .
\end{aligned}
$$

Thus we deduce the desired result.

If $\rho$ and $\tau$ denote as before we have the maps $\left(\begin{array}{cc}\Sigma \rho & C \Sigma \rho \\ C \Sigma \rho & C^{2} \Sigma \rho\end{array}\right): \Pi_{A, B, C} \rightarrow \Pi_{B, C, A}$, $\left(\begin{array}{ll}\Sigma \tau & C \Sigma \tau \\ C \Sigma \tau & C^{2} \Sigma \tau\end{array}\right): \Pi_{A, B, C} \rightarrow \Pi_{C, A, B}$, where $\Pi_{A, B, C}, \Pi_{C, A, B}$ are maps

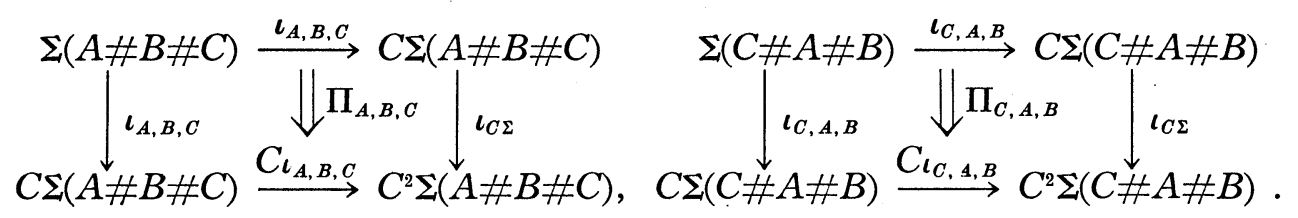

Corollary (5.6). Let $A, B$ and $C$ be suspensions.

(a) If $\alpha \in \pi_{1}(\Sigma A, k), \beta \in \pi_{1}(\Sigma B, k)$ and $\gamma \in \pi(\Sigma C, X)$, then $\left[\alpha,[\beta, \gamma]_{1}\right]_{2}+(\Sigma \rho, C \Sigma \rho)^{*}\left[\beta,[\gamma, \alpha]_{1}\right]_{2}+(\Sigma \tau, C \Sigma \tau)^{*}\left[\gamma,[\alpha, \beta]_{2}\right]_{1}=0$.

(b) If $\alpha \in \pi_{1}(\Sigma A, k), \beta \in \pi_{1}(\Sigma B, k)$ and $\gamma \in \pi_{1}(\Sigma C, k)$, then $\left[\alpha,[\beta, \gamma]_{2}\right]_{2}+(\Sigma \rho, C \Sigma \rho)^{*}\left[\beta,[\gamma, \alpha]_{2}\right]_{2}+(\Sigma \tau, C \Sigma \tau)^{*}\left[\gamma,[\alpha, \beta]_{2}\right]_{2}=0$.

(c) If $\alpha \in \pi_{1}(\Sigma A, u), \beta \in \pi_{1}(\Sigma B, v)$ and $\gamma \in \pi(\Sigma C, W)$, then $\left[\alpha,[\beta, \gamma]_{1}\right]_{3}+\left(\begin{array}{ll}\Sigma \rho & C \Sigma \rho \\ C \Sigma \rho & C^{2} \Sigma \rho\end{array}\right)^{*}\left[\beta,[\gamma, \alpha]_{1}\right]_{3}+\left(\begin{array}{ll}\Sigma \tau & C \Sigma \tau \\ C \Sigma \tau & C^{2} \Sigma \tau\end{array}\right)^{*}\left[\gamma,[\alpha, \beta]_{3}\right]_{4}=0$.

(d) If $\alpha \in \pi_{1}(\Sigma A, u), \beta \in \pi_{1}(\Sigma B, u)$ and $\gamma \in \pi_{1}(\Sigma C, v)$, then $\left[\alpha,[\beta, \gamma]_{3}\right]_{5}+\left(\begin{array}{ll}\Sigma \rho & C \Sigma \rho \\ C \Sigma \rho & C^{2} \Sigma \rho\end{array}\right)^{*}\left[\beta,[\gamma, \alpha]_{3}\right]_{5}+\left(\begin{array}{ll}\Sigma \tau & C \Sigma \tau \\ C \Sigma \tau & C^{2} \Sigma \tau\end{array}\right)^{*}\left[\gamma,[\alpha, \beta]_{2}\right]_{3}=0$.

(e ) If $\alpha \in \pi_{2}(\Sigma A, \Phi), \beta \in \pi(\Sigma B, W)$ and $\gamma \in \pi(\Sigma C, W)$, then

$$
[\alpha,[\beta, \gamma]]_{4}+\left(\begin{array}{ll}
\Sigma \rho & C \Sigma \rho \\
C \Sigma \rho & C^{2} \Sigma \rho
\end{array}\right)^{*}\left[\beta,[\gamma, \alpha]_{4}\right]_{4}+\left(\begin{array}{ll}
\Sigma \tau & C \Sigma \tau \\
C \Sigma \tau & C^{2} \Sigma \tau
\end{array}\right)^{*}\left[\gamma,[\alpha, \beta]_{4}\right]_{4}=0 .
$$

(f) If $\alpha \in \pi_{2}(\Sigma A, \Phi), \beta \in \pi_{1}(\Sigma B, u)$ and $\gamma \in \pi(\Sigma C, W)$, then $\left[\alpha,[\beta, \gamma]_{1}\right]_{5}+\left(\begin{array}{ll}\Sigma \rho & C \Sigma \rho \\ C \Sigma \rho & C^{2} \Sigma \rho\end{array}\right)^{*}\left[\beta,[\gamma, \alpha]_{4}\right]_{5}+\left(\begin{array}{ll}\Sigma \tau & C \Sigma \tau \\ C \Sigma \tau & C^{2} \Sigma \tau\end{array}\right)^{*}\left[\gamma,[\alpha, \beta]_{5}\right]_{4}=0$. 
(g) If $\alpha \in \pi_{2}(\Sigma A, \Phi), \beta \in \pi_{1}(\Sigma B, u)$ and $\gamma \in \pi_{1}(\Sigma C, u)$, then $\left[\alpha,[\beta, \gamma]_{2}\right]_{5}+\left(\begin{array}{ll}\Sigma \rho & C \Sigma \rho \\ C \Sigma \rho & C^{2} \Sigma \rho\end{array}\right)^{*}\left[\beta,[\gamma, \alpha]_{5}\right]_{5}+\left(\begin{array}{cc}\Sigma \tau & C \Sigma \tau \\ C \Sigma \tau & C^{2} \Sigma \tau\end{array}\right)^{*}\left[\gamma,[\alpha, \beta]_{5}\right]_{5}=0$.

(h) If $\alpha \in \pi_{2}(\Sigma A, \Phi), \beta \in \pi_{2}(\Sigma B, \Phi)$ and $\gamma \in \pi(\Sigma C, W)$, then $\left[\alpha,[\beta, \gamma]_{4}\right]_{6}+\left(\begin{array}{ll}\Sigma \rho & C \Sigma \rho \\ C \Sigma \rho & C^{2} \Sigma \rho\end{array}\right)^{*}\left[\beta,[\gamma, \alpha]_{4}\right]_{6}+\left(\begin{array}{ll}\Sigma \tau & C \Sigma \tau \\ C \Sigma \tau & C^{2} \Sigma \tau\end{array}\right)^{*}\left[\gamma,[\alpha, \beta]_{6}\right]_{4}=0$.

(i ) If $\alpha \in \pi_{2}(\Sigma A, \Phi), \beta \in \pi_{2}(\Sigma B, \Phi)$ and $\gamma \in \pi_{1}(\Sigma C, u)$, then $\left.\left[\alpha,[\beta, \gamma]_{5}\right]_{6}\right]+\left(\begin{array}{cc}\Sigma \rho & C \Sigma \rho \\ C \Sigma \rho & C^{2} \Sigma \rho\end{array}\right)^{*}\left[\beta,[\gamma, \alpha]_{5}\right]_{6}+\left(\begin{array}{cc}\Sigma \tau & C \Sigma \tau \\ C \Sigma \tau & C^{2} \Sigma \tau\end{array}\right)^{*}\left[\gamma,[\alpha, \beta]_{6}\right]_{5}=0$

( j ) If $\alpha \in \pi_{2}(\Sigma A, \Phi), \beta \in \pi_{2}(\Sigma B, \Phi)$ and $\gamma \in \pi_{2}(\Sigma C, \Phi)$, then $\left[\alpha,[\beta, \gamma]_{6}\right]_{6}+\left(\begin{array}{ll}\Sigma \rho & C \Sigma \rho \\ C \Sigma \rho & C^{2} \Sigma \rho\end{array}\right)^{*}\left[\beta,[\gamma, \alpha]_{6}\right]_{6}+\left(\begin{array}{ll}\Sigma \tau & C \Sigma \tau \\ C \Sigma \tau & C^{2} \Sigma \tau\end{array}\right)^{*}\left[\gamma,[\alpha, \beta]_{6}\right]_{6}=0$.

PROOF. We shall prove only (e), then the other formulas may be proved similarly when we consider the appropriate universal examples.

Let

$i_{1}: \quad \Sigma A \subset \Sigma A \vee \Sigma B \vee \Sigma C, \quad i_{2}: \quad \Sigma B \subset \Sigma A \vee \Sigma B \vee \Sigma C, \quad i_{3}: \quad \Sigma C \subset \Sigma A \vee \Sigma B \vee \Sigma C$, $i_{1, c}: C \Sigma A \subset C \Sigma A \vee \Sigma B \vee \Sigma C \quad$ and $\quad i_{1, C^{2}}: \quad C^{2} \Sigma A \subset C^{2} \Sigma A \vee \Sigma B \vee \Sigma C$, and let $\alpha=\left\{\left(\begin{array}{ll}f & f^{\prime} \\ f^{\prime \prime} & f^{\prime \prime \prime}\end{array}\right)\right\}, \beta=\{g\}$ and $\gamma=\{h\}$.

Let $\Lambda^{\prime}$ be a map of pair $\left(\iota_{\Sigma A} \bigvee 1_{\Sigma B} \bigvee 1_{\Sigma C}, \iota_{C \Sigma A} \bigvee 1_{\Sigma B} \bigvee 1_{\Sigma C}\right): \iota_{\Sigma A} \bigvee 1_{\Sigma B} \bigvee 1_{\Sigma C}$ $\rightarrow C \iota_{\Sigma A} \vee 1_{\Sigma B} \vee 1_{\Sigma C}:$

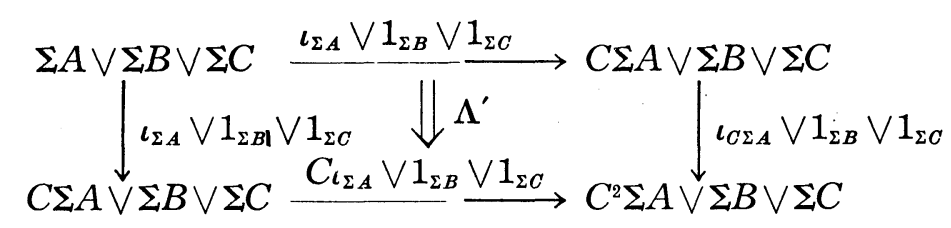

Consider the map: $\left(\begin{array}{ll}p & q \\ r & s\end{array}\right): \Lambda^{\prime} \rightarrow \Phi, \quad$ where $p=\nabla \circ(1 \vee \nabla) \circ(f \vee g \vee h)$, $q=\nabla \circ(1 \vee \nabla) \circ\left(f^{\prime} \vee u g \bigvee u h\right), r=\nabla \circ(1 \vee \nabla) \circ\left(f^{\prime \prime} \vee v g \vee v h\right)$ and $s=\nabla \circ(1 \vee$ $\nabla) \circ\left(f^{\prime \prime} \vee v^{\prime} u g \bigvee u^{\prime} v g\right)$. Let $\iota_{2}=\left\{i_{2}\right\}, \iota_{3}=\left\{i_{3}\right\}$ and $\iota=\left\{\left(\begin{array}{ll}i_{1} & i_{1, G} \\ i_{1, C} & i_{1, C^{2}}\end{array}\right)\right\} \in \pi_{2}\left(\Sigma A, \Lambda^{\prime}\right)$, 
then $\left[\iota,\left[\iota_{2}, \iota_{3}\right]\right]_{4} \in \pi_{2}\left(\Sigma(A \# B \# C), \Lambda^{\prime}\right), \quad\left[\iota_{2},\left[\iota_{3}, \iota\right]_{4}\right]_{4} \in \pi_{2}\left(\Sigma(B \# C \# A), \Lambda^{\prime}\right), \quad\left[\iota_{3},[\iota\right.$, $\left.\left.\iota_{2}\right]_{4}\right]_{4} \in \pi_{2}\left(\Sigma(C \# A \# B), \Lambda^{\prime}\right)$.

We now shall prove the following formula

$$
\left[\iota,\left[\iota_{2}, \iota_{3}\right]\right]_{4}+\left(\begin{array}{ll}
\Sigma \rho & C \Sigma \rho \\
C \Sigma \rho & C^{2} \Sigma \rho
\end{array}\right)^{*}\left[\iota_{2},\left[\iota_{3}, \iota\right]_{4}\right]_{4}+\left(\begin{array}{ll}
\Sigma \tau & C \Sigma \tau \\
C \Sigma \tau & C^{2} \Sigma \tau
\end{array}\right)^{*}\left[\iota_{3},\left[\iota, \iota_{2}\right]_{4}\right]_{4}=0 .
$$

In the homotopy sequence

$$
\begin{aligned}
\longrightarrow & \pi_{2}\left(\Sigma \Lambda, \Lambda^{\prime}\right) \stackrel{\partial_{\Lambda^{\prime}}}{\longrightarrow} \pi_{1}\left(\Sigma \Lambda, \iota_{\Sigma A} \vee 1_{\Sigma B} \vee 1_{\Sigma C}\right) \\
& \stackrel{\left(\iota_{\Sigma A} \bigvee 1_{\Sigma B} \bigvee 1_{\Sigma C}, \iota_{C \Sigma A} \bigvee 1_{\Sigma B} \bigvee 1_{\Sigma C}\right)_{*}}{\longrightarrow} \pi_{1}\left(\Sigma \Lambda, C \iota_{\Sigma A} \bigvee 1_{\Sigma B} \bigvee 1_{\Sigma C}\right),
\end{aligned}
$$

where $\Sigma \Lambda=\Sigma(A \# B \# C), \partial_{\Lambda^{\prime}}$ is an isomorphism since $\pi_{n}\left(\Sigma \Lambda, C \iota_{\Sigma A} \vee 1_{\Sigma B} \vee 1_{\Sigma C}\right)$ $=0, n \geqq 1$. Then we obtain

$$
\begin{aligned}
\partial_{\Lambda^{\prime}}\left(\left[\iota,\left[\iota_{2}, \iota_{3}\right]\right]_{4}+\left(\begin{array}{cc}
\Sigma \sigma & C \Sigma \sigma \\
C \Sigma \sigma & C^{2} \Sigma \sigma
\end{array}\right)^{*}\left[\iota_{2},\left[\iota_{3}, \iota\right]_{4}\right]_{4}+\left(\begin{array}{ll}
\Sigma \tau & C \Sigma \tau \\
C \Sigma \tau & C^{2} \Sigma \tau
\end{array}\right)^{*}\left[\iota_{3},\left[\iota, \iota_{2}\right]_{4}\right]_{4}\right) \\
\quad=\left[\partial_{\Lambda^{\prime} \iota},\left[\iota_{2}, \iota_{3}\right]\right]_{1}+(\Sigma \sigma, C \Sigma \sigma)^{*} \partial_{\Lambda^{\prime}}\left[\iota_{2},\left[\iota_{3}, \iota\right]_{4}\right]_{4}+(\Sigma \tau, C \Sigma \tau)^{*} \partial_{\Lambda^{\prime}}\left[\iota_{3},\left[\iota, \iota_{2}\right]_{4}\right]_{4} \\
\quad=\left[\partial_{\Lambda^{\prime}},\left[\iota_{2}, \iota_{3}\right]\right]_{1}+(\Sigma \sigma, C \Sigma \sigma)^{*}\left[\iota_{2},\left[\iota_{3}, \partial_{\Lambda^{\prime}}\right]_{1}\right]_{1}+(\Sigma \tau, C \Sigma \tau)^{*}\left[\iota_{3},\left[\partial_{\Lambda^{\prime}} \iota, \iota_{2}\right]_{1}\right]_{1} \\
\quad=0 \text { (by (5.5)). }
\end{aligned}
$$

Since $\partial_{\mathbf{A}^{\prime}}$ is the isomorphism we obtain (5.6.1).

By using (5.6.1) we have

$$
\begin{gathered}
\left(\begin{array}{ll}
p & q \\
r & s
\end{array}\right)_{*}\left(\left[\iota,\left[\iota_{2}, \mu_{3}\right]\right]_{4}+\left(\begin{array}{ll}
\Sigma \sigma & C \Sigma \sigma \\
C \Sigma \sigma & C^{2} \Sigma \sigma
\end{array}\right)^{*}\left[\iota_{2},\left[\iota_{3}, \iota\right]_{4}\right]_{4}+\left(\begin{array}{ll}
\Sigma \tau & C \Sigma \tau \\
C \Sigma \tau & C^{2} \Sigma \tau
\end{array}\right)^{*}\left[\iota_{3},\left[\iota, \iota_{2}\right]_{4}\right]_{4}\right) \\
=\left[\left(\begin{array}{cc}
p & q \\
r & s
\end{array}\right)_{*} \iota, p_{*}\left[\iota_{2}, \iota_{3}\right]\right]_{4}+\left(\begin{array}{cc}
\Sigma \sigma & C \Sigma \sigma \\
C \Sigma \sigma & C^{2} \Sigma \sigma
\end{array}\right)^{*}\left(\begin{array}{cc}
p & q \\
r & s
\end{array}\right)_{*}\left[\iota_{2},\left[\iota_{3}, \iota_{4}\right]_{4}\right]_{4} \\
+\left(\begin{array}{ll}
\Sigma \tau & C \Sigma \tau \\
C \Sigma \tau & C^{2} \Sigma \tau
\end{array}\right)^{*}\left(\begin{array}{cc}
p & q \\
r & s
\end{array}\right)_{*}\left[\iota_{3},\left[\iota, \iota_{2}\right]_{4}\right]_{4} \\
=\left[\alpha,\left[p_{*} \iota_{2}, p_{*} \iota_{3}\right]\right]_{4}+\left(\begin{array}{ll}
\Sigma \sigma & C \Sigma \sigma \\
C \Sigma \sigma & C^{2} \Sigma \sigma
\end{array}\right)^{*}\left[p_{*},\left[p_{*} \iota_{3},\left(\begin{array}{ll}
p & q \\
r & s
\end{array}\right)_{*} \iota\right]_{4}\right]_{4} \\
+\left(\begin{array}{ll}
\Sigma \tau & C \Sigma \tau \\
C \Sigma \tau & C^{2} \Sigma \tau
\end{array}\right)^{*}\left[p_{*},\left[\left(\begin{array}{ll}
p & q \\
r & s
\end{array}\right)_{*} \iota, p_{*} \iota_{2}\right]_{4}\right]_{4}
\end{gathered}
$$




$$
\begin{aligned}
& =[\alpha,[\beta, \gamma]]_{4}+\left(\begin{array}{cc}
\Sigma \sigma & C \Sigma \sigma \\
C \Sigma \sigma & C^{2} \Sigma \sigma
\end{array}\right)^{*}\left[\beta,[\gamma, \alpha]_{4}\right]_{4}+\left(\begin{array}{ll}
\Sigma \tau & C \Sigma \tau \\
C \Sigma \tau & C^{2} \Sigma \tau
\end{array}\right)^{*}\left[\gamma,[\alpha, \beta]_{4}\right]_{4} \\
& =0 .
\end{aligned}
$$

Thus we deduce the desired formula.

6. The generalized Hopf invariant of a composition element. In this section, we generalize [8; Theorem 1] to the form of Theorem (6.3).

Throughout this section, we assume that all spaces are finite $C W$-complexes. We recall that

$$
\pi\left(\Sigma A, \Sigma X_{1} \vee \Sigma X_{2}\right) \cong \pi\left(\Sigma A, \Sigma X_{1}\right)+\pi\left(\Sigma A, \Sigma X_{2}\right)+\pi_{1}\left(\Sigma A, j_{X}\right)
$$

where $A$ is a suspension space and $j_{X}: \Sigma X_{1} \vee \Sigma X_{2} \subset \Sigma X_{1} \times \Sigma X_{2}, X=X_{1}=X_{2}$. Then $\pi\left(\Sigma A, \Sigma X_{\lambda}\right), \quad \lambda=1,2$, is embedded in $\pi\left(\Sigma A, \Sigma X_{1} \vee \Sigma X_{2}\right)$ by the injection, and $\pi_{1}\left(\Sigma A, j_{X}\right)$, is embedded in $\pi\left(\Sigma A, \Sigma X_{1} \vee \Sigma X_{2}\right)$ by the boundary monomorphism $\partial_{j_{X}}: \pi_{1}\left(\Sigma A, j_{X}\right) \rightarrow \pi\left(\Sigma A, \Sigma X_{1} \vee \Sigma X_{2}\right)$.

Let $\phi_{*}: \pi(\Sigma A, \Sigma X) \rightarrow \pi\left(\Sigma A, \Sigma X_{1} \vee \Sigma X_{2}\right)$ be the homomorphism induced by the structure map $\phi: \Sigma X \rightarrow \Sigma X \bigvee \Sigma X$. If $\iota_{\lambda}$ is the class of the identity map $\Sigma X \rightarrow \Sigma X, \iota_{\lambda} \in \pi(\Sigma X, \Sigma X)$, regarded as embedded in $\pi\left(\Sigma X, \Sigma X_{1} \vee \Sigma X_{2}\right), \lambda=1,2$, and if $\alpha \in \pi(\Sigma A, \Sigma X)$ then we have easily

$$
\phi_{*}(\alpha)=\left(\iota_{1}+\iota_{2}\right) \circ \alpha,
$$

where $\circ$ is the composite operator.

Let $\left\{f_{\lambda}\right\}=\beta_{\lambda} \in \pi(\Sigma A, X), \lambda=1,2$, and let $\left(\beta_{1}, \beta_{2}\right)$ be the class of maps $\Sigma A_{1} \vee \Sigma A_{2} \rightarrow X\left(A_{\lambda}=A, \lambda=1,2\right)$ which agree on $\Sigma A_{\lambda}$ with a representative of $\boldsymbol{\beta}_{\lambda}$, i.e., $\left(\boldsymbol{\beta}_{1}, \boldsymbol{\beta}_{2}\right)=\left\{\nabla \circ\left(f_{1} \vee f_{2}\right)\right\}$.

LEMMA (6.2). If $\gamma \in \pi(\Sigma, A, \Sigma B)$ and $\beta_{\lambda} \in \pi(\Sigma B, \Sigma D)$, then

$$
\left(\beta_{1}+\beta_{2}\right) \circ \gamma=\beta_{1} \circ \gamma+\beta_{2} \circ \gamma+\left(\beta_{1}, \beta_{2}\right) \circ \partial_{j_{B}} \omega \phi_{*}(\gamma)
$$

where $\omega: \pi(\Sigma A, \Sigma B \vee \Sigma B) \rightarrow \pi_{1}\left(\Sigma A, j_{B}\right)$ is the homomorphism such that $\omega \partial_{j_{B}}=1$ [1], and $A$ is a suspension:

Proof is analogous to the proof of Lemma 2 in [8].

Now we consider the generalized Hopf invariant defined by $H^{*}=\varepsilon_{j} \omega \phi_{*}$ :

$$
\pi(\Sigma A, \Sigma B) \longrightarrow \pi(\Sigma A, \Sigma B \vee \Sigma B) \longrightarrow \pi_{1}\left(\Sigma A, j_{B}\right) \longrightarrow \pi_{1}(\Sigma A, \Sigma B \# \Sigma B),
$$

where $A$ is a suspension, and $\varepsilon_{j}$ is the excision homomorphism (c.f. [1]). 
In this section, we have the following theorem:

THEOREM (6.3). Let $\beta \in \pi(\Sigma B, \Sigma D)$ and $\gamma \in \pi(\Sigma A, \Sigma B)$. If $A$ and $B$ are suspensions then

$$
H^{*}(\beta \circ \gamma)=H^{*}(\beta) \circ \Sigma \gamma+(\beta \# \beta) \circ H^{*}(\gamma)
$$

ProOF. By using Lemma (6.2) as in [8] we have

$$
\begin{aligned}
\partial_{j_{D}} \omega \phi_{*}(\beta \circ \gamma)=\partial_{j_{D}} \omega \phi_{*}(\beta) & \circ \gamma+\left(\iota_{1} \circ \beta, \iota_{2} \circ \beta\right) \circ \partial_{j_{B}} \omega \phi_{*}(\gamma) \\
& +\left(\iota_{1} \circ \beta+\iota_{2} \circ \beta, \partial_{j_{D}} \omega \phi_{*}(\beta)\right) \circ \partial_{j_{B}} \omega \phi_{*}(\gamma) .
\end{aligned}
$$

Since $H^{*}=\varepsilon_{j} \omega \phi_{*}$ we obtain

$$
\begin{array}{r}
H^{*}(\beta \circ \gamma)=\varepsilon_{j_{D}} \partial_{j_{D}}^{-1}\left(\partial_{j_{D}} \omega \phi_{*}(\beta) \circ \gamma\right)+\varepsilon_{j_{D}} \partial_{j_{D}}^{-1}\left(\left(\iota_{1} \circ \beta, \iota_{2} \circ \beta\right) \circ \partial_{j_{B}} \omega \phi_{*}(\gamma)\right) \\
+\varepsilon_{j_{D}} \partial_{j_{D}}^{-1}\left(\left(\iota_{1} \circ \beta+\iota_{2} \circ \beta, \partial_{j_{D}} \omega \phi_{*}(\beta)\right) \circ \partial_{j_{B}} \omega \phi_{*}(\gamma)\right)
\end{array}
$$

(a) We shall prove $\varepsilon_{j D} \partial_{j D}^{-1}\left(\partial_{j D} \omega \phi_{*}(\beta) \circ \gamma\right)=H^{*}(\beta) \circ \Sigma \gamma$.

Let $\gamma=\{f\} \in \pi(\Sigma A, \Sigma B)$, and we consider the commutative diagram

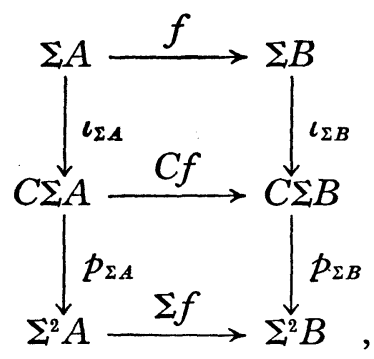

where $p_{\Sigma A}$ and $p_{\Sigma B}$ are projections. Then we have the following diagram

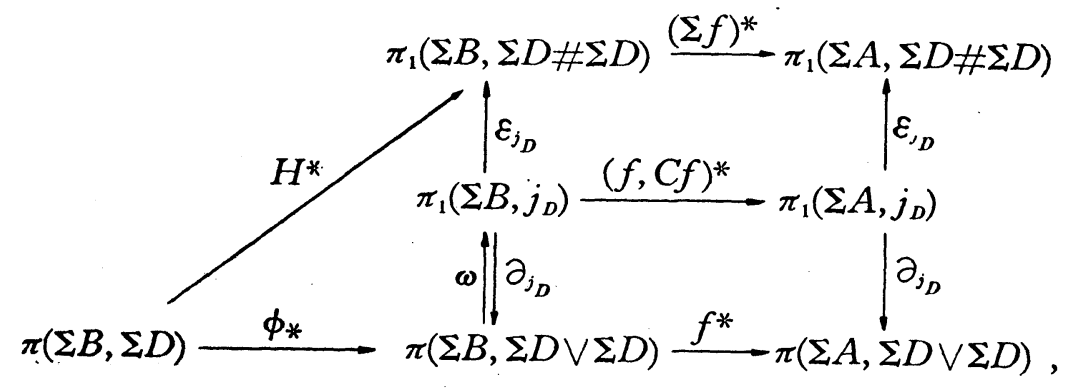


where each $\partial_{j_{D}}$ is a monomorphism and $\omega$ is an epimorphism. For $\beta \in \pi(\Sigma B, \Sigma D)$ we have $(\Sigma f)^{*} H^{*}(\beta)=(\Sigma f)^{*}\left(\varepsilon_{j_{D}} \omega \phi_{*}(\beta)\right)=\varepsilon_{j_{D}}(f, C f)^{*}\left(\omega \phi_{*}(\beta)\right)$ $=\varepsilon_{j_{D}} \partial_{j_{D}}^{-1} f^{*} \partial_{j_{D}}\left(\omega \phi_{*}(\beta)\right)$. Hence we obtain $H^{*}(\beta) \circ \Sigma \gamma=\varepsilon_{j_{D}} \partial_{j_{D}}^{-1}\left(\partial_{j_{D}} \omega \phi_{*}(\beta) \circ \gamma\right)$.

(b) We shall prove $\left.\varepsilon_{j_{D}} \partial_{j_{D}}^{-1}\left(\iota_{1} \circ \beta, \iota_{2} \circ \beta\right) \circ \partial_{j_{B}} \omega \phi_{*}(\gamma)\right)=(\beta \# \beta) \circ H^{*}(\gamma)$.

Let $\beta=\{g\} \in \pi(\Sigma B, \Sigma D)$, then $\left(\iota_{1} \circ \beta, \iota_{2} \circ \beta\right)=\beta \vee \beta=\{g \vee g\} \in \pi(\Sigma B \vee \Sigma B$, $\Sigma D \vee \Sigma D)$, and we consider the commutative diagram

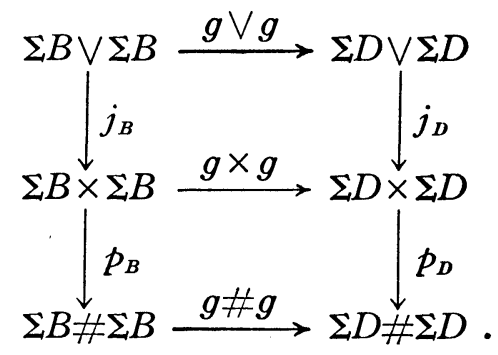

We have the following diagram

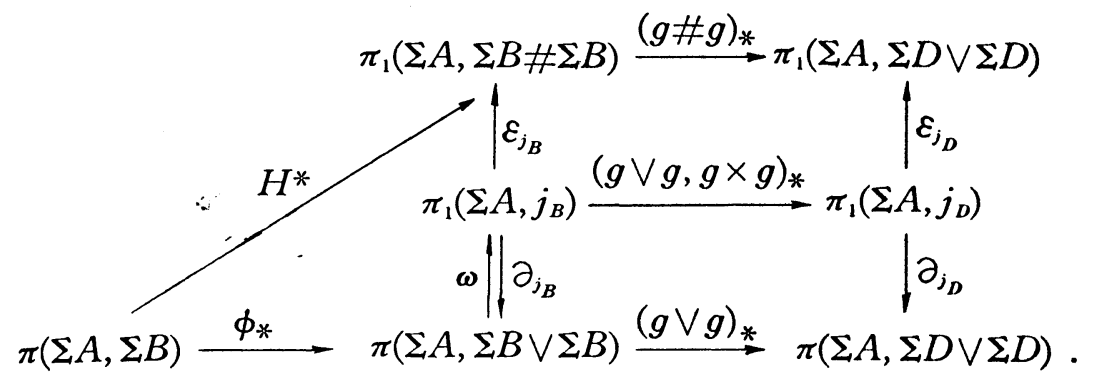

For $\gamma \in \pi(\Sigma A, \Sigma B)$ we obtain $\varepsilon_{j_{D}} \partial_{j_{D}}^{-1}\left((g \vee g)_{*}\left(\partial_{j_{B}} \omega \phi_{*}(\gamma)\right)=\varepsilon_{j_{D}}(g \vee g, g \times g)_{*} \omega \phi_{*}(\gamma)\right.$ $=(g \# g)_{*} \varepsilon_{j_{D}} \omega \phi_{*}(\gamma)=(g \# g)_{*} H^{*}(\gamma)$. Thus we deduce $\varepsilon_{j_{D}} \partial_{j_{D}}^{-1}\left(\left(\iota_{1} \circ \beta, \iota_{2} \circ \beta\right)\right.$ $\left.\circ \partial_{j_{B}} \omega \phi_{*}(\gamma)\right)=(\beta \# \beta) \circ H^{*}(\gamma)$.

Finally we shall prove that

(c) $\varepsilon_{j_{D}} \partial_{j_{D}}^{-1}\left(\left(\iota_{1} \circ \beta+\iota_{2} \circ \beta, \partial_{j_{D}} \omega \phi_{*}(\beta)\right) \circ \partial_{j_{B}} \omega \phi_{*}(\gamma)\right)=0$.

It is sufficient to prove that $\varepsilon_{j_{D}} \partial_{j_{D}}^{-1}\left(\beta_{1}+\beta_{2}, \partial_{j_{D}} \omega \phi_{*}(\beta)\right)_{*}\left(\partial_{j_{B}} \omega \phi_{*}(\gamma)\right)=0$, where $\beta_{1}=\iota_{1} \circ \beta, \beta_{2}=\iota_{2} \circ \beta$. We set $\xi=\beta_{1}+\beta_{2}, \eta=\partial_{j_{D}} \omega \phi_{*}(\beta)$ and $\zeta=\partial_{j_{B}} \omega \phi_{*}(\gamma)$ and again we denote by $\xi, \eta$ and $\zeta$ these representatives. Since $\eta=\partial_{j_{D}} \omega \phi_{*}(\beta)$ $\epsilon \pi(\Sigma B, \Sigma D \vee \Sigma D)$ for $\beta \in \pi(\Sigma B, \Sigma D)$ we have $j_{D *}(\eta)=0$ and hence $j_{D} \eta \simeq *$, and we obtain the following diagram 


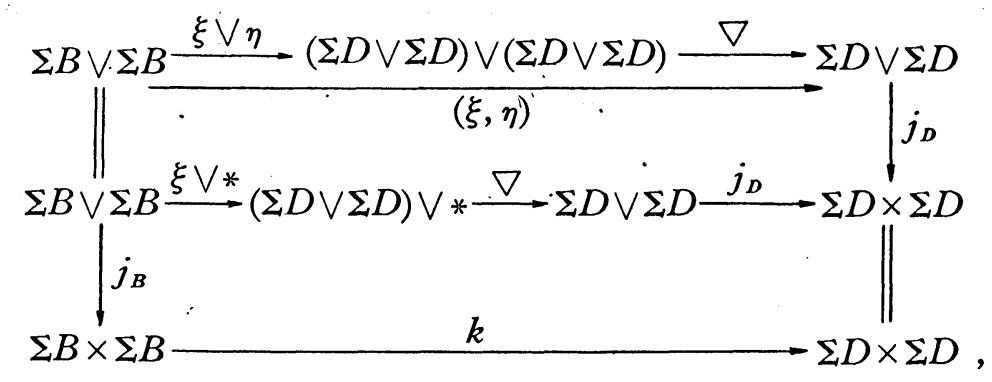

the upper diagram is homotopy commutative and the low diagram is commutative, where $k$ is defined by $k(x, y)=j_{D}(\xi, *)(x \vee y)$ for $x, y \in \Sigma B$. Then $j_{D}(\xi, \eta) \simeq k j_{B}$, and since $j_{B}$ is a cofibration there exists $k^{\prime}: \Sigma B \times \Sigma B$ $\rightarrow \Sigma D \times \Sigma D$ such that $j_{D}(\xi, \eta)=k^{\prime} j_{B}$, and we have the following commutative diagram

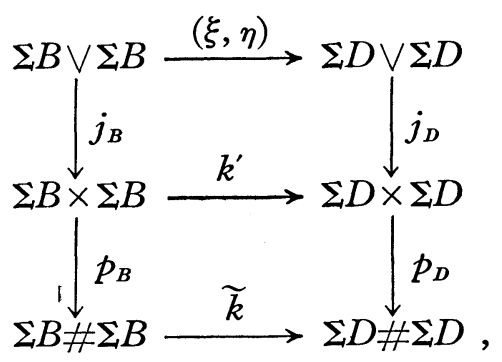

where $\widetilde{k}$ is determined by $(\xi, \eta)$ and $k^{\prime}$. Then we obtain the diagram

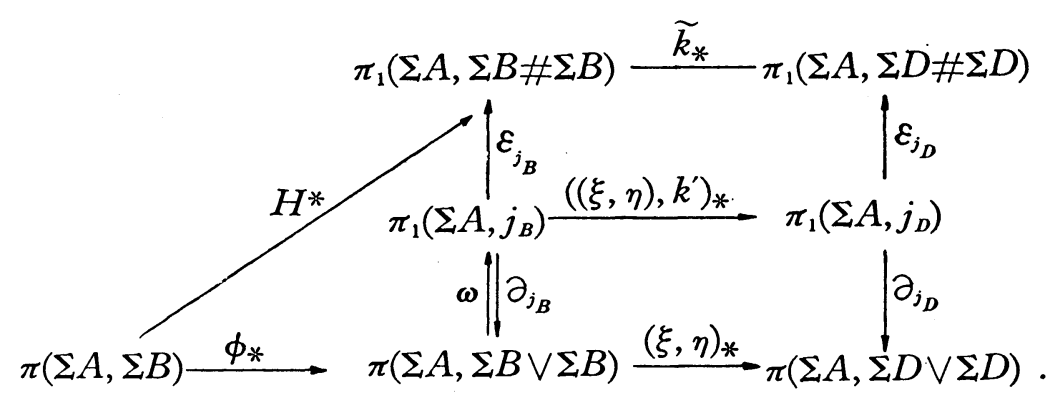

Consider the commutative diagram 


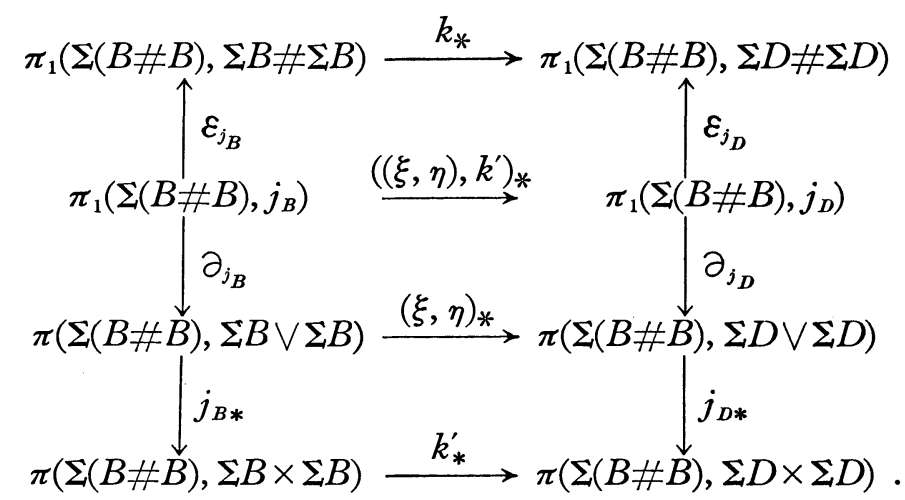

If we choose $\{\bar{\rho} \bar{h}, \rho h)\} \in \pi_{1}\left(\Sigma(B \# B), j_{B}\right)$ then $\varepsilon_{j_{B}}\{(\bar{\rho} \bar{h}, \rho h)\}=\{t\} \in \pi_{1}(\Sigma(B \# B)$, $\Sigma B \# \Sigma B)$ where $t: \Sigma^{2}(B \# B) \rightarrow \Sigma B \# \Sigma B$ is a homotopy equivalence (c.f. [1]), and $\partial_{j_{B}}\{(\bar{\rho} \bar{h}, \rho h)\}=\{\bar{\rho} \bar{h}\}=\theta$. Since $j_{B *}(\theta)=0$ ([12; Theorem 2.2]) we have $0=k_{*}^{\prime} j_{B *}(\theta)=j_{D *}(\xi, \eta)_{*}(\theta)=j_{D *}[\xi, \eta]$, and hence there exists $\alpha \in \pi_{1}(\Sigma(B \# B)$, $\left.j_{D}\right)$ such that $\partial_{j_{D}} \alpha=[\xi, \eta]$.

Next we consider the GWP $\left[\xi, \omega \phi_{*}(\beta)\right]_{1} \in \pi_{1}\left(\Sigma(B \# B), j_{D}\right)$ of $\xi \in \pi(\Sigma B$, $\Sigma D \vee \Sigma D)$ and $\omega \phi_{*}(\beta) \in \pi_{1}\left(\Sigma B, j_{D}\right)$, then $\partial_{j_{D}}\left[\xi, \omega \phi_{*}(\beta)\right]_{1}=\left[\xi, \partial_{j_{D}} \omega \phi_{*}(\beta)\right]=[\xi, \eta]$ $=\partial_{j_{D}} \alpha$. Since $\partial_{j_{D}}$ is a monomorphism we have

$$
\alpha=\left[\xi, \omega \varphi_{*}(\beta)\right]_{1} .
$$

By the commutativity of (6.3.2)

$$
\begin{aligned}
\varepsilon_{j_{D}}\left((\xi, \eta), k_{*}^{\prime}\{(\bar{\rho} \bar{h}, \rho h)\}\right. & =\varepsilon_{j_{D}} \partial_{j_{D}}^{-1}(\xi, \eta)_{*} \partial_{j_{B}}\{(\bar{\rho} \bar{h}, \rho h)\} \\
& =\varepsilon_{j_{D}} \partial_{j_{D}}^{-1}(\xi, \eta)_{*}(\theta) \\
& =\varepsilon_{j_{D}} \partial_{j_{D}}^{-1}[\xi, \eta] \\
& =\varepsilon_{j_{D}} \alpha \\
& =\varepsilon_{j_{D}}\left[\xi, \omega \phi_{*} \beta\right]_{1} \quad(\text { by }(6.3 .3)) .
\end{aligned}
$$

While

$$
\begin{aligned}
\varepsilon_{j_{D}}\left((\xi, \eta), k^{\prime}\right)_{*}\{(\bar{\rho} \bar{h}, \rho h)\} & \left.=\widetilde{k}_{*} \varepsilon_{j_{B}}\{\bar{\rho} \bar{h}, \rho h)\right\} \\
& =\widetilde{k}_{*}\{t\}
\end{aligned}
$$

thus we have $\varepsilon_{j_{D}}\left[\xi, \omega \phi_{*} \beta\right]_{1}=\widetilde{k}_{*}\{t\}$. The excision homomorphism $\varepsilon_{j_{D}}$ : $\pi_{1}\left(\Sigma(B \# B), j_{D}\right) \rightarrow \pi_{1}(\Sigma(B \# B), \Sigma D \# \Sigma D)$ is represented as follows : 


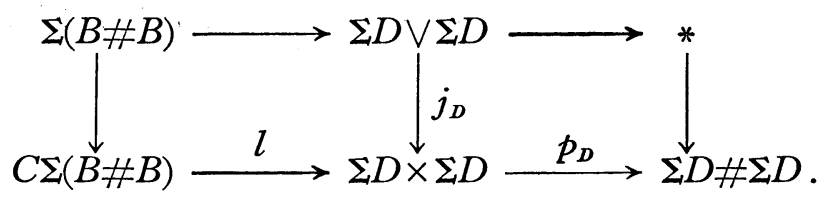

Hence $\varepsilon_{j_{D}}\left[\xi, \omega \phi_{*}(\beta)\right]_{1}=\left(*, p_{D} l\right)_{*}\left[\xi, \omega \phi_{*}(\beta)\right]_{1}=\left[*,\left(*, p_{D} l\right)_{*} \omega \phi_{*}(\beta)\right]_{1}=0$. Therefore $\widetilde{k}_{*}\{t\}=0$. For any element $\{f\} \in \pi(\Sigma A, \Sigma B \# \Sigma B)$, since $f \simeq t\left(t^{-1} f\right)$ we have $\widetilde{k}_{*}\{f\}=\{(\widetilde{k} f)\}=\left\{(\widetilde{k} t)\left(t^{-1} f\right)\right\}=0$. Hence, by the commutativity of (6.3.1),

$$
\varepsilon_{j_{D}} \partial_{j_{D}}^{-1}(\xi, \eta)_{*} \partial_{j_{B}} \omega \phi_{*}(\gamma)=\widetilde{k}_{*} \varepsilon_{j_{B}} \omega \phi_{*}(\gamma)=\widetilde{k}_{*} H^{*}(\gamma)=0 .
$$

Therefore by (a), (b) and (c) we deduce

$$
H^{*}(\beta \circ \gamma)=H^{*}(\beta) \circ \Sigma \gamma+(\beta \# \beta) \circ H^{*}(\gamma) .
$$

In [1] we defined the generalized Hopf invariant as follows: Assume that $\Sigma X$ is $(n-1)$-connected and $A$ is a suspension space. Then

(i) If $\operatorname{dim} . \Sigma A \leqq 3 n-3$, then

$$
\begin{array}{r}
H=\bar{h}_{*}^{-1} \partial(\bar{\rho}, \rho)_{*}^{-1} \omega \phi_{*}: \pi(\Sigma A, \Sigma X) \longrightarrow \pi(\Sigma A, \Sigma X \bigvee \Sigma X) \longrightarrow \pi_{1}(\Sigma A, j) \\
\longrightarrow \pi_{1}\left(\Sigma A, j_{Q}\right) \longrightarrow \pi(\Sigma A, Q) \longrightarrow \pi(\Sigma A, \Sigma(X \# X)) .
\end{array}
$$

(ii) If $\operatorname{dim} . \Sigma A \leqq 2(2 n-2)$, then

$$
\begin{aligned}
H= & \Sigma^{-1} t_{*} \varepsilon_{j} \omega \phi_{*}: \pi(\Sigma A, \Sigma X) \longrightarrow \pi(\Sigma A, \Sigma X \vee \Sigma X) \longrightarrow \pi_{1}(\Sigma A, j) \\
& \longrightarrow \pi_{1}(\Sigma A, \Sigma X \# \Sigma X) \longrightarrow \pi_{1}\left(\Sigma A, \Sigma^{2}(X \# X)\right) \longrightarrow \pi(\Sigma A, \Sigma(X \# X)) .
\end{aligned}
$$

If $\operatorname{dim} \Sigma A \leqq 3 n-3, H$ in (ii) equal to $H$ in (i) (c.f. [1]), and hence if we identify $\pi_{1}(\Sigma A, \Sigma X \# \Sigma X)$ with $\pi_{1}\left(\Sigma A, \Sigma^{2}(X \# X)\right)$ under the isomorphism $t_{*}^{-1}$, the Hopf invariant $H$ is denoted $\Sigma^{-1} \varepsilon_{j} \omega \phi_{*}$ and $H^{*}=\Sigma H$.

The following properties are shown easily from (6.3), where $A$ and $B$ are suspensions, and $\beta \in \pi(\Sigma B, \Sigma X), \gamma \in \pi(\Sigma A, \Sigma B)$ :

(6.4) If $\Sigma B$ is (n-1)-connected, $\Sigma X$ is (s-1)-connected, and if $\operatorname{dim} \Sigma B$ $\leqq 3 n-3,3 s-3, \operatorname{dim} \Sigma A \leqq 3 s-3$ and $H(\gamma)=0$, then

$$
H(\beta \circ \gamma)=H(\beta) \circ \gamma
$$


(6.5) If $B$ is (n-2)-connected, $X$ is (s-2)-connected, and if $\operatorname{dim} \Sigma A \leqq 3 s-3$ $\leqq 3 n-3$ then for $\beta^{\prime} \in \pi(B, X)$ we have

$$
H\left(\Sigma, \beta^{\prime} \circ \gamma\right)=\Sigma\left(\beta^{\prime} \# \beta^{\prime}\right) \circ H(\gamma)
$$

(6. 6) If $\gamma \in \Sigma \pi(A, B)$, then

$$
H^{*}(\beta \circ \gamma)=H^{*}(\beta) \circ \Sigma \gamma .
$$

(6. 7) If $\Sigma B$ is (n-1)-connected, $\Sigma X$ is (s-1)-connected, and if $\Sigma A \leqq 4 n-4$, $\operatorname{dim} \Sigma B \leqq 4 s-4$, then

$$
H^{*}(\beta \circ \gamma)=\Sigma H(\beta) \circ \Sigma \gamma+(\beta \# \beta) \circ \Sigma H(\gamma) .
$$

(6. 8) If $\beta \in \Sigma \pi(B, X)$, then

$$
H^{*}(\beta \circ \gamma)=(\beta \# \beta) \circ H^{*}(\gamma)
$$

\section{REFERENCES}

[1] H. ANDo AND K. Tsuchida, On excision and its applications, Sci. Rep. Hirosaki Univ., 13(1966), 92-103.

[2] M. ARKowITZ, The generalized Whitehead product, Pacific Journ. of Math., 12(1962), 7-23.

[3] M. ARkowITZ, Homotopy product for $H$-spaces, Michigan Math. Journ., 10(1963), 1-9.

[4] A. L. Blakers AND W.S. MASSEY; Products in homotopy theory, Ann. of Math., 58 (1953), 295-324.

[5] B. ECKMANN AND P. J. HILTON, Homtopy groups of maps and exact sequences, Comm. Math. Helv., 34(1960), 271-304.

[6] K. A. HARDIE, On a construction of E. C. Zeeman, Journ. London Math. Soc., 35(1960), $452-464$.

[ 7 ] P. J. Hilton, Suspension theorem and generalized Hopf invariant, Proc. London Math. Soc., Ser. 3, 2(1951), 462-493.

[8] P. J. Hilton, Hopf invariant of a composition element, Journ. London Math. Soc., 29 (1954), 165-171.

[9] P. J. HiltoN, On the homotopy groups of the union of spheres, Journ. London Math. Soc., 30 (1955), 154-172.

[10] P. J. HILtoN, Homotopy theory and duality, Mimeographed Notes, Cornell University, 1959.

[11] S. T.HU, A group multiplication for relative homotopy groups, Journ. London Math. Soc., 22(1947), 61-67.

[12] G. T. PORTER, Higher order Whitehead products, Topology, 3(1965), 123-135.

[13] G. W. WhiteheAD, Generalization of the Hopf invariant, Ann. of Math. (2), 51(1950), 192-237.

[14] G. W. WHITWHAD, On mappings into group-like spaces, Comm. Math. Helv., 28(1954), 320-328.

[15] J. H. C. WhITEHEAD, On adding relation to homotopy group, Ann. of Math., 42(1941), 409-429.

MATHMATICAL INSTITUTE

AKITA UNIVERSITY

AKITA. JAPAN 